UNIVERSIDADE DE SÃO PAULO

INSTITUTO DE FÍSICA

\title{
Quantização da partícula não relativística em espaços curvos como superfícies do $\mathbb{R}^{\mathrm{n}}$
}

Maria Fernanda Araujo de Resende

Orientador: Prof. Dr. Dmitry Maximovitch Gitman - IFUSP

Dissertação apresentada ao Instituto de Física da USP para a obtenção do título de Mestre em Ciências

\section{Banca Examinadora}

Prof. Dr. Dmitry Maximovitch Gitman - IFUSP

Prof. Dr. Josif Frenkel - IFUSP

Prof. Dr. Daniel Victor Tausk - IME-USP

São Paulo 


\section{FICHA CATALOGRÁFICA}

\section{Preparada pelo Serviço de Biblioteca e Informação}

\section{do Instituto de Física da Universidade de São Paulo}

Resende, Maria Fernanda Araujo de

Quantização da partícula não relativística em espaços curvos como superfícies do $\mathbb{R}^{\mathrm{n}}$. - São Paulo, 2011

Dissertação (Mestrado) - Universidade de São Paulo.

Instituto de Física, Departamento de Física Nuclear.

Orientador: Prof. Dr. Dmitry Maximovitch Gitman

Área de Concentração: Física

Unitermos: 1. Física teórica; 2. Covariância;

3. Quantização ; 4. Mergulho isométrico;

5. Ordenamento de operadores.

USP/IF/SBI-091/2011 
Dedicado a mim mesmo, pois sem eu nada disso teria ocorrido. 


\section{Agradecimentos}

Muitas pessoas se envolveram, diretamente ou não, ao longo do desenvolvimento do trabalho aqui presente e, de certo, algumas destas eventualmente não serão mencionadas devido a prováveis problemas nas sinapses que ocorrem em meu cérebro. Deste modo, antes de qualquer agradecimento, antecipo meus sinceros pedidos de desculpas aos que não encontrarem seus nomes listados abaixo.

Inicio meus agradecimentos aos meus pais, Fernando Monteiro de Resende e Celsina Jacinta de Araujo, pelo apoio incondicional que me foi dado, especialmente por minha mãe, perante o conhecimento da importância que meus estudos têm em minha vida: graças ao seu árduo e honesto trabalho, assim como à toda sua dedicação, pude construir minha formação acadêmica e pessoal.

Mencionando minha formação acadêmica, também direciono outro profundo agradecimento a todos os professores que contribuíram de algum modo para sua construção. Entre eles, um especial cabe aos Profs. Drs. Antonio Domingues dos Santos, Artur Hideyuki Tomita, Dmitry Maximovitch Gitman, Mikiya Muramatsu e Renata Zukanovich Funchal, ambos da Universidade de São Paulo. Com efeito, também agradeço aos Profs. Drs. Adilson José da Silva, Daniel Victor Tausk, Josif Frenkel, Paulo Teotonio Sobrinho e Rosa Maria dos Santos Barreiro Chaves, da Universidade de São Paulo, e ao Prof. Dr. Alberto Vazquez Saa, da Universidade de Campinas, por todas as discussões, sugestões e aconselhamentos, assim como ao Prof. Dr. Naohisa Ogawa, do Hokkaido Institute of Technology, pela sua gentil e preciosa colaboração no desenvolvimento de alguns dos cálculos mais vitais deste trabalho: é sempre bom saber que existem pessoas com sabedoria, simplicidade e boa vontade de serem úteis ao próximo.

Agradecimentos também cabem aos meus amigos do Instituto de Matemática e Estatística da USP e dos diversos departamentos do Instituto de Física da USP, dentre os quais destaco meus "companheiros de jornada": Bárbara Sayuri Ashino, Carlos Antonio Filho, Eduardo Roberto de Lascio, Everton Santos Medeiros, Fabiola Diacenco Xavier, Fabrício Marques do Carmo, Felipe Augusto Vilaverde Custódio, Gabriela Cotrim de Moraes, Leandro Daros Gama, Leandro Saccoletto, Priscilla Iastremski, Rafael Oliveira Suigh, Robson Aparecido Figueiredo, Rodrigo Figueiredo, 
Rodrigo Fresneda, Simone Camargo Trippe e Ulisses Alves Maciel Neto.

Para concluir, faço um honesto agradecimento aos CNPq e Capes por todo o suporte financeiro. 


\section{Resumo}

Neste trabalho estudamos o problema relacionado à construção de uma teoria quântica para uma partícula, se movendo não relativisticamente num espaço curvo, tratado como uma subvariedade de outro Euclideano, talvez dando maior ênfase ao aspecto geométrico envolvido nesta abordagem, uma vez que os demais trabalhos relacionados ao mesmo tema não o fazem.

Além de mostrarmos que o consequente uso de uma teoria de sistemas vinculados não contribui para remover as ambiguidades da formulação quântica, relacionados diretamente ao ordenamento de operadores, também apresentamos, através de uma quantização específica feita sob a prescrição de Dirac, elementos que permitem não apenas construir um formalismo quântico covariante, mas também liberto de qualquer "correção quântica".

Em adição, fazemos alguns comentários gerais no que se refere às outras abordagens clássicas possíveis para o mesmo problema, intentando construir teorias quânticas associadas ao sistema sob consideração. 


\section{Abstract}

In this work we study the problem related to the construction of a quantum theory for a particle, moving non-relativistically in a curved space, treated as submanifold of the other Euclidean, maybe putting more emphasis on the geometric aspect envolved in this approach, since the rest of the works related to the subject do not.

Besides showing that the consequent use of a theory of constrained systems not contributes for remove the ambiguities in the quantum formulation, related directly to the ordering of operators, we also showing, through a specific quantization made in the prescription of Dirac, elements that offers resources not only to construct a covariant quantum formalism, but also free from any "quantum correction".

In addition, we make some general comments in relation to other classical approaches possible for the same problem, attempting to build quantum theories associated with the system under consideration. 


\section{Sumário}

1 Introdução 1

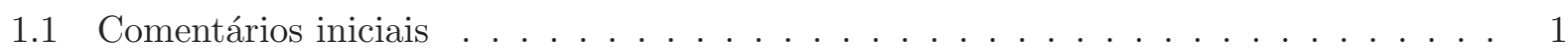

1.2 A estruturação do trabalho . . . . . . . . . . . . . . . . . . 4

1.3 Sobre a notação utilizada . . . . . . . . . . . . . . . . . . . . 6

$\begin{array}{llr}2 & \text { Espaços Riemannianos imersos no } \mathbb{R}^{\mathrm{N}} & 7\end{array}$

2.1 Considerações iniciais $\ldots \ldots \ldots \ldots \ldots \ldots \ldots \ldots$

2.2 Sobre variedades diferenciáveis . . . . . . . . . . . . . . . . . 8

2.3 Imersões, mergulhos e subvariedades $\ldots \ldots \ldots \ldots \ldots \ldots$

2.4 A imersão de variedades Riemannianas no $\mathbb{R}^{\mathbb{N}} \ldots \ldots \ldots \ldots \ldots \ldots$

2.4.1 Algumas particularidades . . . . . . . . . . . . . . . . . . 12

2.5 Características associadas às imersões isométricas . . . . . . . . . . . . . . . . . 14

$\begin{array}{lll}3 & \text { O problema da partícula } & 17\end{array}$

3.1 Considerações Lagrangianas . . . . . . . . . . . . . . . . . . . . 17

3.2 O formalismo Hamiltoniano . . . . . . . . . . . . . . . . . . 18

3.3 As equações de consistência . . . . . . . . . . . . . . . . . . . . . . 19

3.3 .1 Sobre o conjunto de vínculos . . . . . . . . . . . . . . . . . 21

3.4 Algumas relações clássicas . . . . . . . . . . . . . . . . . . . . . . . . 22

3.5 Observações importantes . . . . . . . . . . . . . . . . . . . . . . . . . . 24

4 O processo de quantização $\quad 26$

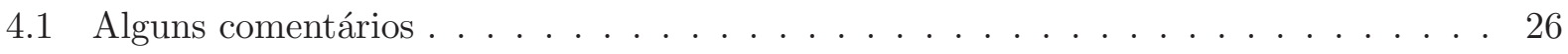

4.2 O ordenamento de operadores . . . . . . . . . . . . . . . 27

4.3 Relações canônicas de comutação . . . . . . . . . . . . . . . . . . . . . . 30 
4.3.1 Um cálculo honestamente enfadonho . . . . . . . . . . . . . . . . . . . . 32

4.4 Sobre o operador Hamiltoniano . . . . . . . . . . . . . . . . . . . 37

4.4 .1 Outro cálculo quase enfadonho . . . . . . . . . . . . . . 39

4.4 .2 Algumas considerações . . . . . . . . . . . . . . . . . . . . . . . . . 41

4.5 A geometria do potencial quântico . . . . . . . . . . . . . . . . . . 42

5 Uma formulação alternativa $\quad 46$

5.1 Considerações para a equivalência . . . . . . . . . . . . . . . . . . . . 46

5.2 Consequências imediatas . . . . . . . . . . . . . . . . . . . . . . 47

$5.2 .1 \quad$ Observações pertinentes . . . . . . . . . . . . . . . . . 50

5.2 .2 Uma consideração adicional . . . . . . . . . . . . . . . . . . . . . . . 51

5.3 A construção de uma teoria quântica . . . . . . . . . . . . . . . . . . . . . 56

6 Conclusões e comentários $\quad 59$

$\begin{array}{ll}\text { A Considerações Hamiltonianas } & 63\end{array}$

A.1 Algumas observações importantes . . . . . . . . . . . . . . . . . . . . 63

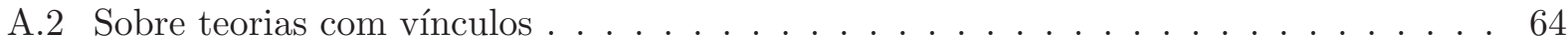

A.2.1 Informações relevantes . . . . . . . . . . . . . . . . . . . 65

A.3 Parênteses de Dirac e propriedades . . . . . . . . . . . . . . . 66

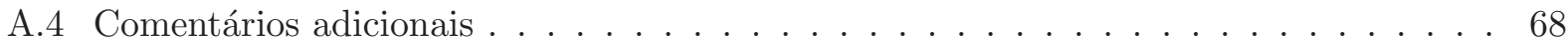

$\begin{array}{ll}\text { B Uma observação importante } & 70\end{array}$

$\begin{array}{ll}\text { C Algumas informações relevantes } & 72\end{array}$

C.1 Uma observação quântica adicional . . . . . . . . . . . . . . . . . . . . . . 72

C.2 Outras informações geométricas . . . . . . . . . . . . . . . . . . . 73

C.2.1 Sobre a métrica e a curvatura . . . . . . . . . . . . . . . . . 73

C.2.2 Alguns resultados específicos . . . . . . . . . . . . . . . . 74

$\begin{array}{ll}\text { Referências Bibliográficas } & 75\end{array}$ 


\section{Capítulo 1}

\section{Introdução}

\subsection{Comentários iniciais}

Para a construção de uma teoria capaz de descrever um sistema físico qualquer, devemos ter alguns cuidados. O primeiro, e mais fundamental deles, é garantir que os resultados obtidos dos modelos e considerações nela resumidos sejam verificáveis experimentalmente, dentro do domínio onde a mesma se define. Já o segundo cuidado está relacionado a eventual existência de uma outra teoria física, também passível de descrever o mesmo sistema quando definida para outro domínio arbitrário: a nova teoria não pode contradizer os resultados da primeira já bem estabelecida. Tratase do chamado príncipio da correspondência: sempre que existirem duas teorias para um mesmo sistema físico, uma delas deve se "aproximar" da outra dando resultados que, entre certos limites, coincidem [1].

No caso específico da criação de uma teoria quântica para um sistema físico com representação clássica, o procedimento padrão adotado para isso consiste em "promover" todos os parâmetros responsáveis pela descrição Hamiltoniana do sistema a operadores autoadjuntos, atuantes nos vetores de algum espaço de Hilbert $\mathfrak{H}$ necessariamente complexo, local onde são descritos todos os estados quânticos relacionados ao sistema $[2,3]$. Por consequência, trata-se de uma "promoção" que ocorre com todas as funções definidas neste ambiente clássico, identificado com algum fibrado cotangente $T^{*} \mathcal{M}$ provido de uma estrutura simplética salvo algumas exceções [4], e relacionadas diretamente aos observáveis da teoria física. Com efeito, podemos dizer que quantizar um sistema físico é o mesmo que construir uma correspondência entre estas mesmas funções e operadores autoadjuntos em $\mathfrak{H}^{1}$.

\footnotetext{
${ }^{1}$ Não entraremos em maiores detalhes matemáticos relacionados ao assunto. No entanto eles podem ser encontrados, por exemplo, em [5] e particularmente nos seus textos: Note on Quantization, de P. Deligne; e Elementary Introduction to Quantum Field Theory, de L. Fadeev e notas de L. Jeffrey.
} 
Considerando sistemas físicos cuja dinâmica clássica se descreve num $T^{*} \mathcal{M}$ munido de uma álgebra simplética descrita por $\omega=(q, p)$, a quantização destes fica definida por meio de uma representação $\mathrm{Q}$, de todas as funções clássicas $\mathcal{A} \in C^{\mathrm{k}}\left(T^{*} \mathcal{M}\right)$ suprarreferidas como operadores autoadjuntos $\hat{A}=\mathrm{Q}(\mathcal{A})$ em algum $\mathfrak{H}$, e tal que $[6,7]$

(i) $\mathrm{Q}(\mathcal{A}+\mathcal{B})=\mathrm{Q}(\mathcal{A})+\mathrm{Q}(\mathcal{B})$,

(ii) $\mathrm{Q}(\lambda \mathcal{A})=\lambda \mathrm{Q}(\mathcal{A})$, onde $\lambda$ é um número complexo arbitrário,

(iii) $[\mathrm{Q}(\mathcal{A}), \mathrm{Q}(\mathcal{B})]=i \hbar\{\mathcal{A}, \mathcal{B}\}$,

(iv) $\mathrm{Q}(1)=\hat{I}$, sendo $\hat{I}$ o operador identidade, com

(v) $\mathbf{Q}(q)$ e $\mathbf{Q}(p)$ possuindo representações irredutíveis no $\mathfrak{H}$.

Por se dizer, o último item pode ser retraduzido especificamente como [8]

$$
\left[\hat{q}^{\mu}, \hat{q}^{\nu}\right]=\left[\hat{p}_{\mu}, \hat{p}_{\nu}\right]=0 \quad, \quad\left[\hat{q}^{\mu}, \hat{p}_{\nu}\right]=i \hbar \hat{\delta}_{\nu}^{\mu}
$$

diante do condicionamento (iii) e pelo uso dos chamados parênteses de Poisson, definidos no Apêndice A. $1^{2}$.

Em linhas gerais, podemos dizer que o processo de quantização se define associando a cada observável $\mathcal{A}$ um único operador $\hat{A}=\mathrm{Q}(\mathcal{A})$, sendo $\mathrm{Q}: T^{*} \mathcal{M} \rightarrow \mathfrak{H}$ uma correspondência entre álgebras de Lie: ou seja, enquanto os observáveis clássicos formam uma álgebra estruturada pelos parênteses de Poisson, os operadores quânticos formam outra, também de Lie, onde os comutadores figuram necessariamente como os seus parênteses.

Entretanto a tarefa de construir teorias quânticas desta maneira nem sempre é simples, afinal trata-se de uma prescrição no mínimo não específica, face ao estabelecimento de um $\hat{A}=\mathrm{Q}(\mathcal{A})$ autoadjunto quando $\mathcal{A}$ se expressa necessariamente por produtos entre os parâmetros canonicamente conjugados $q$ e $p$ do formalismo clássico Hamiltoniano, uma vez que a consequente "promoção" destes podem remeter a um conjunto de operadores não comutativos. Logo a presença de tais produtos em qualquer função clássica impõe necessariamente que escolhas devem ser feitas, no que

\footnotetext{
${ }^{2}$ Aliás, recomendamos a leitura do Apêndice A como um todo, não apenas para maiores informações sobre este ponto em específico, mas principalmente ao que se refere à representação clássica de sistemas em termos de outros parâmetros não necessariamente simpléticos. Isso será de extrema importância ao que apresentamos no Capítulo 3 , por exemplo.
} 
se refere ao ordenamento dos operadores quânticos relacionados às variáveis canonicamente conjugadas, para a definição de um operador autoadjunto a ela associado, implicando automaticamente na eventual não unicidade de uma teoria quântica para um sistema físico ${ }^{3}$.

Um bom exemplo deste "problema" se relaciona aos sistemas físicos constituídos por partículas massivas em movimento não relativístico num espaço Riemanniano $\mathcal{M}$ com D dimensões. Segundo o formalismo clássico, ao considerarmos $\mathcal{M}$ parametrizado por $q=\left(q^{1}, \ldots, q^{\mathrm{D}}\right)$, podemos descrever um destes sistemas através da Lagrangiana $L: T \mathcal{M} \rightarrow \mathbb{R}$ dada por

$$
L=L(q, \dot{q})=\frac{m}{2} g_{\mu \nu}(q) \dot{q}^{\mu} \dot{q}^{\nu}-\mathcal{V}(q)
$$

onde $\dot{q}^{\mu}$ e $g_{\mu \nu}$ denotam as respectivas componentes associadas à velocidade e ao tensor métrico Riemanniano $[11,12]$, e $\mathcal{V}(q)$ algum potencial ao qual o sistema adicionalmente se submete.

Apesar desta Lagrangiana ser não singular, perante o relacionamento isomórfico entre os fibrados tangente $T \mathcal{M}$ e cotangente $T^{*} \mathcal{M}$, e trazer por meio de uma transformação de Legendre [13] a função $H: T^{*} \mathcal{M} \rightarrow \mathbb{R}$, dada por

$$
H=H(p, q)=\frac{1}{2 m} g^{\mu \nu}(q) p_{\mu} p_{\nu}+\mathcal{V}(q)
$$

como a Hamiltoniana associada ao mesmo sistema, a construção de uma teoria quântica para este nitidamente não pode ser feita de modo único, visto que os operadores $\hat{q}$ e $\hat{p}$ devem obedecer às relações (1.1), afinal produtos entre $\hat{p}_{\mu}$ e $g^{\mu \nu}(\hat{q})$ não serão necessariamente comutativos ${ }^{4}$, implicando na não unicidade um operador $\hat{H}$ autoadjunto.

Umas das primeiras tentativas em quantizar este sistema em específico foi feita por DeWitt [14]: a sua escolha foi construir um Hamiltoniano autoadjunto pelo simples ordenamento simétrico de

\footnotetext{
${ }^{3}$ Também não entraremos nos detalhes deste assunto, porém apenas a título de informação, no ano de 1946 H. J. Groenwald demonstrou em [9] que a busca por um mapa não apenas específico, mas "aceitável" para a quantização de sistemas clássicos definidos em espaços planos é inteiramente inútil, uma vez que a álgebra de Poisson, constituída por todos os polinômios definidos no $T^{*} \mathbb{R}^{\mathrm{N}}$, não pode ser quantizada a ponto de obtermos um conjunto de operadores simétricos, e portanto autoadjuntos em $\mathfrak{H}$ : essa plena quantização, se assim podemos dizer, ocorre apenas quando libertamos o processo de quantização do condicionamento (v) acima listado. Cinco anos mais tarde, L. van Hove generalizou este mesmo resultado demonstrando em [10] que, a priori, é impossível quantizar todos os observáveis clássicos que descrevem-se por meio dos polinômios já mencionados, quaisquer sejam as escolhas tomadas para isso e em respeito as regras supracitadas: no caso uma quantização consistente se dá apenas quando restringimo-nos a uma subálgebra do $C^{\infty}\left(T^{*} \mathbb{R}^{\mathbb{N}}\right)$.

${ }^{4} \mathrm{~A}$ menos que expressão da métrica independa dos parâmetros envolvidos, logo não se referindo ao caso de um espaço curvo [12].
} 
operadores que, no nosso caso, se resume $\mathrm{em}^{5}$

$$
\hat{H}=H(\hat{p}, \hat{q})=\frac{1}{2 m} \hat{p}_{\mu} g^{\mu \nu}(\hat{q}) \hat{p}_{\nu}+\mathcal{V}(\hat{q})
$$

Embora essa formulação possua o mesmo limite clássico (1.2), trata-se de uma escolha que, por

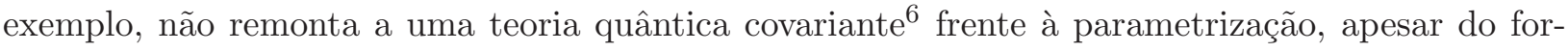
malismo clássico Hamiltoniano o ser pelas chamadas transformações de ponto, expressas por

$$
q^{\prime \mu}=q^{\prime \mu}(q) \quad, \quad p_{\mu}^{\prime}=\frac{\partial q^{\nu}}{\partial q^{\prime \mu}} p_{\nu}
$$

face não somente à covariância do tensor métrico, mas à invariância das equações de movimento pelas mesmas transformações. Em particular, a não covariância associada a (1.3) é devida, tão somente, à presença de um termo dependente dos símbolos de Christoffel ao substituirmos as expressões

$$
\hat{q}^{\mu}=q^{\mu}, \hat{p}_{\mu}=-i \hbar \mathrm{g}^{-\frac{1}{4}} \partial_{\mu} \mathrm{g}^{\frac{1}{4}}
$$

escolhidas por DeWitt ${ }^{7}$. Explicitamente temos

$$
\hat{H}=-\frac{\hbar^{2}}{2 m} \Delta+\mathcal{V}(q)-\hbar^{2} K(q)
$$

onde $\Delta=\mathrm{g}^{-\frac{1}{4}} \hat{p}_{\mu} \sqrt{\mathrm{g}} \hat{g}^{\mu \nu} \hat{p}_{\nu} \mathrm{g}^{-\frac{1}{4}}$ é o operador de Laplace-Beltrami associado ao espaço $\mathcal{M}$ e

$$
K(q)=\frac{1}{4 m} g^{\mu \nu}\left(\Gamma_{\alpha \mu, \nu}^{\alpha}-\Gamma_{\mu \nu}^{\alpha} \Gamma_{\beta \alpha}^{\beta}-\frac{1}{2} \Gamma_{\alpha \mu}^{\alpha} \Gamma_{\beta \nu}^{\beta}\right)
$$

\subsection{A estruturação do trabalho}

De acordo com a expressão (1.5), espaços Riemannianos nos quais teorias quânticas, covariantes frente à parametrização, podem ser definidas unicamente são aqueles cujas componentes dos tensores métricos associados independem dos parâmetros adotados. Assim, perante à observação de que espaços Euclideanos satisfazem a este requerimento, o objetivo do presente trabalho é apresentar

\footnotetext{
${ }^{5}$ No caso original de DeWitt foi considerado uma partícula livre.

${ }^{6} \mathrm{O}$ termo covariância está relacionado à dependência exclusiva de uma aplicação qualquer apenas dos termos pertencentes à geometria intrínseca do ambiente onde a mesma aplicação se define [15]. Sob o ponto de vista físico este termo se relaciona, por consequência, ao aspecto de todas as leis físicas serem expressas da mesma maneira, quaisquer sejam as parametrizações adotadas para isso [16].

${ }^{7}$ Devemos observar que trata-se de uma escolha diretamente associada às observações feitas no Apêndice C.1, as quais relacionam-se à medida Riemanniana aqui presente.
} 
um procedimento alternativo para a quantização de sistemas físicos definidos em espaços curvos, com respaudo no fato de que quaisquer espaços Riemannianos podem ser considerados como subvariedades de outros Euclideanos. Deste modo, ao invés de lidarmos com um sistema "livre" num ambiente curvo, considerá-lo-emos restrito a uma superfície regular de outro, Euclideano e dimensionalmente maior ${ }^{8}$, para avaliar se este processo em específico oferece recursos para libertar o formalismo quântico dos problemas suprarreferidos.

Com efeito desta característica, antes de qualquer consideração relacionada ao problema físico em pauta, o próximo capítulo será devotado às justificativas que permitem o mergulho isométrico de espaços Riemannianos em outros Euclideanos acontecer, uma vez que elas são inexistentes nos demais trabalhos relacionados ao problema físico, apesar da sua indubitável importância. Isso será feito através da prévia apresentação de alguns conceitos fundamentais diretamente relacionados ao que chamamos de variedades diferenciáveis e à geometria de espaços Riemannianos [22, 23].

Após todos estes comentários, iniciamos no Capítulo 3 a apresentação do problema resumido nestas notas com um estudo clássico relacionado à dinâmica do sistema físico, agora vinculado a uma superfície regular de um espaço Euclideano. Além de demonstrarmos que essa abordagem alternativa remonta a uma teoria clássica em total equivalência com a definida originalmente num espaço Riemanniano qualquer, também mostramos que o mesmo procedimento não livra o processo de quantização dos problemas relacionados à ambiguidade na definição dos operadores autoadjuntos.

Aliás, uma vez que as considerações do Capítulo 3 constroem necessariamente uma teoria Lagrangiana singular, procedemos em acordo ao método de quantização proposto por Dirac [24] para a obtenção de uma teoria física capaz de descrever o sistema quanticamente: os estudos relacionados a esse procedimento estão resumidos no Capítulo 4 onde, diferentemente dos trabalhos anteriores relacionados ao mesmo tema, apresentamos alguns comentários gerais, tangentes à definição de operadores quânticos nesta situação, perante às questões de autoadjunticidade.

Por consequência do estabelecimento desta teoria, realizamos, no mesmo capítulo, uma análise geométrica sobre o operador Hamiltoniano assim obtido, intentando avaliar a existência de algum fator, diretamente associado ao mergulho de um espaço curvo em outro Euclideano, passível de tornar a mesma teoria covariante frente à parametrização intrínseca adotada: a novidade obtida é que, para os processos de mergulhos mínimos, definidos ao término do Capítulo 2, isso não apenas ocorre, como também liberta a teoria de quaisquer correções quânticas.

Frente à constatação de que não existe apenas um único formalismo Lagrangiano representa-

\footnotetext{
${ }^{8}$ Trata-se de um método intensamente estudado por diversos autores no início dos anos 90 [17, 18, 19, 20, 21].
} 
tivo para este sistema físico, diante das outras escolhas possíveis para a implementação de vínculos, tecemos no Capítulo 5 algumas considerações alternativas no que tange à definição clássica Hamiltoniana do mesmo sistema, demonstrando que elas também remetem a uma teoria clássica equivalente às apresentadas anteriormente. Por consequência, e baseados em todo o material acumulado, discutimos brevemente algumas questões diretamente relacionadas à criação de uma teoria quântica equivalente à mencionada no Capítulo 4.

Para encerrar, além de sintetizar todos os resultados obtidos num último capítulo, completamos este trabalho com três apêndices intuindo esclarecer algumas questões que fugiram ao enredo do texto principal, seja por eventuais "desvios" de assunto ou mesmo por simples sobrecarga de informações, mas que indubitavelmente complementam o trabalho em aspectos fundamentais.

\subsection{Sobre a notação utilizada}

Visando a boa compreensão do trabalho apresentado nas próximas páginas, seria interessante alertar o leitor sobre algumas das escolhas relacionadas à notação empregada. Entretanto, como entendemos que a maior parte das observações referentes a isso já estão bem postas ao longo do texto principal, talvez a única que necessite um esclarecimento preliminar se refira aos somatórios, visto ela já ter sido utilizada no início deste capítulo ${ }^{9}$.

Por questões de simplicidade e "leveza notacional" por exemplo, adotamos a convenção de Einstein no que compete a eles: ou seja, sempre que índices se repetirem num mesmo termo, pertencente a uma igualdade qualquer, estará subentendido que um somatório está presente. Em particular, ao longo destas notas, os índices gregos contabilizam uma quantidade D de termos, enquanto os latinos maiúsculos e minúsculos varrem um espectro natural referente a $\mathrm{N}$ e $\mathrm{D}+\mathrm{N}$ termos respectivamente. Assim para evitar confusão, reservamos aos índices D e N a exclusiva tarefa de identificações dimensionais ${ }^{10}$.

Aliás, devido a esta escolha em particular, talvez caiba ressaltar que o símbolo $\mathbb{R}^{\mathbb{N}}$ não se refere ao conjunto de todas as aplicações $f: \mathrm{N} \rightarrow \mathbb{R}$, por exemplo, e sim a um espaço Euclideano N-dimensional. Ou seja,

$$
\mathbb{R}^{\mathrm{N}}=\left\{\left(x^{1}, \ldots, x^{\mathrm{N}}\right): x^{\mathrm{A}} \in \mathbb{R}\right\}
$$

\footnotetext{
${ }^{9}$ Vide por exemplo (1.2), (1.3), e a Lagrangiana apresentada imediatamente antes destas mesmas expressões.

${ }^{10}$ Apenas como exemplos, vale citar: $\mu=1, \ldots, \mathrm{D} ; \mathrm{A}=1, \ldots, \mathrm{N} ; \mathrm{e} \mathrm{j}=1, \ldots, \mathrm{D}+\mathrm{N}$.
} 


\section{Capítulo 2}

\section{Espaços Riemannianos imersos no $\mathbb{R}^{\mathrm{N}}$}

\subsection{Considerações iniciais}

O objetivo fundamental deste capítulo é mostrar como espaços Riemannianos podem ser considerados subespaços de outros, em particular dos Euclideanos. Trata-se de uma possibilidade diretamente associada ao fato destes serem exemplos do que subentendemos por variedades diferenciáveis.

De um modo geral o conceito de variedade ${ }^{1}$ é muito semelhante ao de uma superfície regular, sendo talvez nele inspirado conforme tornar-se-á claro nas próximas linhas. Explicitamente, ao considerarmos $U$ e $V$ como os respectivos abertos dos $\mathbb{R}^{\mathrm{D}}$ e $\mathbb{R}^{\mathrm{N}}$, uma superfície regular com $\mathrm{D}$ dimensões se define como um subconjunto $\mathcal{S}$ no mesmo espaço quando, em cada ponto de $\mathrm{V}$,

(S1) existir um homeomorfismo diferenciável $\phi: \mathrm{U} \rightarrow \mathrm{V} \cap \mathcal{S}$, sendo

(S2) a aplicação $d \phi_{\mathrm{q}}: \mathbb{R}^{\mathrm{D}} \rightarrow \mathbb{R}^{\mathrm{N}}$ injetiva em todo $\mathrm{q} \in \mathrm{U}$.

Em particular, a propriedade (S1) compõe esta definição simplesmente para garantir a existência de um plano tangente em todos os pontos de $\mathcal{S}$, uma vez que ela exclui possíveis autointersecções em $\mathcal{S}$. Já a propriedade $(\mathrm{S} 2)$ serve para impor as chamadas condições de regularidade em $\mathcal{S}$. Aliás devemos notar que, pelo uso de uma representação matricial sobre bases canônicas, dada por

$$
d \phi_{\mathrm{q}}=\left(\frac{\partial x^{\mathrm{A}}}{\partial u^{\mathrm{j}}}\right)
$$

a mesma propriedade (S2) se reformula com a simples exigência dos vetores coluna de (2.1) serem linearmente independentes, visto a injetividade de $d \phi_{\mathbf{q}}$.

\footnotetext{
${ }^{1}$ Por questões de simplicidade, eventualmente omitiremos o termo "diferenciável" associado às variedades.
} 
Apenas para constar, as aplicações $\phi_{\alpha}: \mathrm{U}_{\alpha} \rightarrow \mathrm{V}_{\alpha} \cap \mathcal{S}$ tais como as mencionadas em (S1) são as $\operatorname{cartas}^{2}$ : através delas qualquer aberto $\mathrm{V}_{\alpha}$ na superfície se descreve em função dos parâmetros de outro aberto $U_{\alpha}$ pertencente ao $\mathbb{R}^{\mathrm{N}}$. Em especial, caso $\phi_{\alpha}$ e $\phi_{\beta}$ remontem a um $\phi_{\alpha}\left(\mathrm{U}_{\alpha}\right) \cap$ $\phi_{\beta}\left(\mathrm{U}_{\beta}\right)=\mathrm{W}$ não vazio, prova-se que $\phi_{\alpha}^{-1} \circ \phi_{\beta}: \phi_{\beta}^{-1}(\mathrm{~W}) \rightarrow \mathbb{R}^{\mathrm{D}}$ e $\phi_{\beta}^{-1} \circ \phi_{\alpha}: \phi_{\alpha}^{-1}(\mathrm{~W}) \rightarrow \mathbb{R}^{\mathrm{D}}$ são diferenciáveis [15]. Deste modo, uma superfície regular pode ser vista como uma união de abertos do $\mathbb{R}^{\mathbb{N}}$ tais que, para aqueles que possuem intersecções não vazias uns com os outros, a transição entre eles ocorre de maneira diferenciável.

Um bom exemplo de superfície regular surge com a ajuda de um aberto $U$ pertencente ao $\mathbb{R}^{\mathrm{D}+\mathrm{N}}$ e uma função $f: U \rightarrow \mathbb{R}^{\mathrm{D}}$ de classe $C^{\mathrm{k}}$. Explicitamente a superfície se define pelo aberto não vazio

$$
\mathcal{S}=\left\{x \in \mathrm{U}: f(x)=c \text { e } f^{\prime}: \mathbb{R}^{\mathrm{D}+\mathrm{N}} \rightarrow \mathbb{R}^{\mathrm{D}} \text { é sobrejetora }\right\}
$$

em $f^{-1}(c)^{3}$.

\subsection{Sobre variedades diferenciáveis}

O conceito resumido por variedade diferenciável se refere a um conjunto $\mathcal{M}$, considerado um espaço topológico de Hausdorff ${ }^{4}$ com base enumerável [26], e uma família de biunívocas $\phi_{\alpha}: \cup_{\alpha} \subset$ $\mathbb{R}^{\mathrm{D}} \rightarrow \mathcal{M}$ tais que

(V1) $\bigcup_{\alpha} \phi_{\alpha}\left(\mathrm{U}_{\alpha}\right)=\mathcal{M}$

(V2) quando $\phi_{\alpha}\left(\mathrm{U}_{\alpha}\right) \cap \phi_{\beta}\left(\mathrm{U}_{\beta}\right)=\mathrm{W}$ é não vazio, necessariamente $\phi_{\alpha}^{-1}(\mathrm{~W})$ e $\phi_{\beta}^{-1}(\mathrm{~W})$ são abertos pertencentes ao $\mathbb{R}^{\mathrm{N}}$ e $\phi_{\beta}^{-1} \circ \phi_{\alpha}$ é um difeomorfismo ${ }^{5}$; e

(V3) o atlas $\left\{\left(\mathrm{U}_{\alpha}, \phi_{\alpha}\right)\right\}$ é maximal em relação (V1) e (V2).

De um modo geral, podemos interpretar $\mathcal{M}$ como um espaço D-dimensional que localmente se comporta como um Euclideano, seja do ponto de vista topológico ou mesmo por sua estrutura diferenciável, que é constituída pela família $\left\{\left(\mathrm{U}_{\alpha}, \phi_{\alpha}\right)\right\}$. Aliás, uma vez que esta mesma estrutura satisfaz as propriedades (V1) e (V2), uma topologia $\tau_{\mathcal{M}}$ é naturalmente induzida em $\mathcal{M}$ : basta adotar $\mathrm{V}$ como um aberto de $\mathcal{M}$ sempre que $\phi_{\alpha}^{-1}\left(\mathrm{~V} \cap \phi_{\alpha}\left(\mathrm{U}_{\alpha}\right)\right)$ for um aberto do $\mathbb{R}^{\mathrm{N}}$ para qualquer

\footnotetext{
${ }^{2}$ Convém frisar que, ao contrário da terminologia adotada, os índices rotulando cartas e abertos não se associam a qualquer dimensionalidade: eles apenas indicam que geralmente várias cartas são necessárias para a descrição da superfície como um todo.

${ }^{3}$ Para maiores detalhes sobre a caracterização de $\mathcal{S}$ como superfície, vide o Apêndice B.

${ }^{4}$ Isso quer dizer que, para pontos distintos $q$ e $q^{\prime}$ pertencentes ao $\mathcal{M}$, existem as respectivas vizinhanças $V_{q}$ e $V_{q}$, tais que $\mathrm{V}_{\mathrm{q}} \cap \mathrm{V}_{\mathrm{q}^{\prime}}=\varnothing$.

${ }^{5} \mathrm{Ou}$ seja, $\phi_{\beta}^{-1} \circ \phi_{\alpha}$ é uma bijeção diferenciável com inversa também diferenciável.
} 
$\phi_{\alpha}$. Logo $\tau_{\mathcal{M}}$ fica definida tomando $\phi_{\alpha}\left(\mathrm{U}_{\alpha}\right)$ como abertos de $\mathcal{M}$, sendo $\phi_{\alpha}: \mathrm{U}_{\alpha} \subset \mathbb{R}^{\mathrm{D}} \rightarrow \mathcal{M}$ aplicações diferenciáveis.

Nestas variedades podemos definir curvas através de aplicações diferenciáveis $\alpha:(-\varepsilon, \varepsilon) \rightarrow \mathcal{M}$ e vetores tangentes, em cada ponto de $\mathcal{M}$, considerando que eles tangenciam algumas dessas curvas num $t=0$ arbitrário. Explicitamente, para uma carta $\phi: U \subset \mathbb{R}^{\mathrm{D}} \rightarrow \mathcal{M}$, o vetor tangente fica expresso por

$$
\dot{\alpha}(0)=\left.\dot{a}^{\mu} \frac{\partial}{\partial q^{\mu}}\right|_{t=0}
$$

visto $\left\{\partial / \partial q^{1}, \ldots, \partial / \partial q^{\mathrm{D}}\right\}_{t=0}$ ser uma base para o espaço tangente $T_{\mathbf{q}} \mathcal{M}$ quando $t=0$ num ponto q da variedade $\mathcal{M}$.

Aliás, devido às condições listadas acima, percebemos facilmente que quaisquer superfícies regulares no $\mathbb{R}^{\mathrm{N}}$ são exemplos de variedades, dado a propriedade referente à transição entre abertos figurar como um dos seus axiomas. Em verdade, a diferença essencial entre as duas definições apresentadas acima, é a sempre necessária ambientação de uma superfície regular em outro conjunto dimensionalmente maior, enquanto que para uma variedade isso não é preciso [22].

Outro exemplo de variedade é o espaço Euclideano N-dimensional, cuja estrutura é provida pelo atlas $\{(\mathrm{U}, \phi)\}$ de sistema único de coordenadas, dado por $\phi: \mathbb{R}^{\mathrm{N}} \rightarrow \mathbb{R}^{\mathrm{N}}$. Em particular trata-se de uma variedade Riemanniana ${ }^{6}$, uma vez que a sua métrica se dá por $\left\langle e_{\mathrm{A}}, e_{\mathrm{B}}\right\rangle=\delta_{\mathrm{AB}}$, onde $e_{\mathrm{A}}$ são os vetores ortonormais que servem de base para o $\mathbb{R}^{\mathrm{N}}$. A característica interessante da consideração de espaços Euclideanos como variedades é que cada um dos seus abertos admite parametrização não somente por um sistema de coordenadas cartesianas, mas também por outros de coordenadas curvilíneas ${ }^{7}$.

\subsection{Imersões, mergulhos e subvariedades}

Os exemplos de variedades mencionados nos dois últimos parágrafos não foram meramente ilustrativos: eles serviram para apresentar a real possibilidade de variedades serem definidas em outras dimensionalmente maiores, particularmente em espaços Euclideanos, retratando um exemplo do que chamamos de subvariedade, cujo conceito apresentamos a seguir. Entretanto, algumas noções preliminares se fazem necessárias e a principal delas diz respeito às chamadas imersões.

\footnotetext{
${ }^{6}$ Por definição, e de um modo bem simples, uma métrica Riemanniana é uma correspondência que associa, em cada $\mathrm{q} \in \mathcal{M}$, um produto interno no $T_{\mathrm{q}} \mathcal{M}$ que varia de maneira diferenciável.

${ }^{7}$ Para uma demonstração talvez mais detalhada destes mesmos exemplos vide [22], especialmente as páginas 131 e 132. Um exemplo adicional de variedade que também cabe ser mencionado, embora não seja importante ao que segue neste capítulo em específico, é o fibrado tangente, definido por $T \mathcal{M}=\left\{(q, v): \mathrm{q} \in \mathcal{M}, v \in T_{\mathrm{q}} \mathcal{M}\right\}$; para maiores detalhes sobre este fato, remetemos o leitor a [23].
} 
O conceito de imersão de uma variedade $\mathcal{M}$ com $\mathrm{D}$ dimensões em outra $\mathcal{N}$, com $\mathrm{N}$ dimensões extras, fica definido de modo natural desde que a primeira seja regular em todos os seus pontos. Explicitamente uma imersão se define por uma aplicação diferenciável $\varphi: \mathcal{M} \rightarrow \mathcal{N}$, sendo $d \varphi_{\mathrm{q}}$ : $T_{\mathrm{q}} \mathcal{M} \rightarrow T_{\varphi(\mathrm{q})} \mathcal{N}$ injetiva em todos os pontos q de $\mathcal{M}$. Em particular, caso o mesmo processo de imersão traga $\mathcal{M}$ e $\varphi(\mathcal{M}) \subset \mathcal{N}$ como conjuntos homeomorfos, a aplicação $\varphi$ será um mergulho.

Assim, com esses conceitos em mente e pela cômoda consideração das mesmas variedades, diremos que $\mathcal{M}$ será uma subvariedade de $\mathcal{N}$ se [22, 23]

(I) $\mathcal{M}$ estiver contida em $\mathcal{N}$, sendo a última a indutora de uma topologia em $\mathcal{M}$, e a inclusão $i: \mathcal{M} \hookrightarrow \mathcal{N}$ for um mergulho; e

(II) para todo $\mathrm{q} \in \mathcal{M}$ existirem cartas $\phi_{1}: \mathrm{U}_{1} \subset \mathbb{R}^{\mathrm{D}} \rightarrow \mathcal{M}$ e $\phi_{2}: \mathrm{U}_{2} \subset \mathbb{R}^{\mathrm{D}+\mathrm{N}} \rightarrow \mathcal{N}$, com $\phi_{2}^{-1} \circ \phi_{1}: \phi_{1}^{-1}\left(\mathrm{~V}_{1}\right) \rightarrow \mathbb{R}^{\mathrm{D}+\mathrm{N}}$ sendo uma imersão, e $\mathrm{V}_{1}$ e $\mathrm{V}_{2}$ vizinhanças de $\mathrm{q}$ em $\mathcal{M}$ e $\mathcal{N}$ respectivamente ${ }^{8}$.

De um modo geral, o comportamento da subvariedade $\mathcal{M}$ em $\mathcal{N}$ é análogo ao de uma superfície regular num espaço Euclideano. Por se dizer, uma superfície de classe $C^{\mathrm{k}}$ contida no $\mathbb{R}^{\mathrm{D}+\mathrm{N}}$ figura como um exemplo de subvariedade, também de classe $C^{\mathrm{k}}$, do mesmo Euclideano [22].

Outro conceito de extrema importância ao que segue é o de isometria. Para as duas variedades $\mathcal{M}$ e $\mathcal{N}$ em pauta, uma isometria é definida através de qualquer difeomorfismo $\varphi: \mathcal{M} \rightarrow \mathcal{N}$, onde para todo $\mathrm{q} \in \mathcal{M}$ e vetores $u, v \in T_{\mathrm{q}} \mathcal{M}$ temos

$$
\langle u, v\rangle_{\mathrm{q}}=\left\langle d \varphi_{\mathrm{q}}(u), d \varphi_{\mathrm{q}}(v)\right\rangle_{\varphi(\mathrm{q})}
$$

No caso particular de $\varphi$ ser uma imersão e $\mathcal{N}$ Riemanniana, uma estrutura também Riemanniana será induzida em $\mathcal{M}$ através de (2.4), fazendo de $\varphi$ uma imersão isométrica, visto a positividade do produto interno.

Uma das características fundamentais de um processo de imersão isométrica, por exemplo, é a decomposição diferenciável de $T_{\mathrm{q}} \mathcal{N}$, em qualquer ponto q pertencente a $\mathcal{M}$, na soma $\operatorname{direta}^{9}$

$$
T_{\mathrm{q}} \mathcal{N}=T_{\mathrm{q}} \mathcal{M} \oplus\left(T_{\mathrm{q}} \mathcal{M}\right)^{\perp}
$$

sendo $\left(T_{\mathbf{q}} \mathcal{M}\right)^{\perp}$ o complemento ortogonal de $T_{\mathbf{q}} \mathcal{M}$, denotado por espaço normal da imersão em q.

\footnotetext{
${ }^{8}$ Em verdade, a aplicação $\phi_{2}^{-1} \circ \phi_{1}$ será um mergulho visto a indução de uma topologia em $\mathrm{V}_{1}$ por $\mathrm{V}_{2}$.

${ }^{9}$ Por questões de simplicidade, utilizaremos $T_{\mathrm{q}} \mathcal{N}$ ao invés de $T_{\varphi(\mathrm{q})} \mathcal{N}$, uma vez que o processo de imersão traz $\mathrm{q} \in \mathcal{M} \subset \mathcal{N}$.
} 
Devido a esta peculiaridade, qualquer vetor $w \in T_{\mathrm{q}} \mathcal{N}$ é expresso como $w=w^{\|}+w^{\perp}$, onde $w^{\|} \mathrm{e}$ $w^{\perp}$ pertencem a $T_{\mathrm{q}} \mathcal{M}$ e $\left(T_{\mathrm{q}} \mathcal{M}\right)^{\perp}$ respectivamente ${ }^{10}$.

\subsection{A imersão de variedades Riemannianas no $\mathbb{R}^{\mathrm{N}}$}

Apesar de todas as superfícies regulares em espaços Euclideanos serem exemplos de variedades, esse fato não implica necessariamente que variedades são imersas em quaisquer Euclideanos, logo podendo ser consideradas como superfícies regulares. Entre outras coisas, existem algumas condições dimensionais que, quando supridas pelo ambiente Euclideano, permitem às variedades serem nele imersas ou mergulhadas. Uma boa resposta dada neste sentido foi apresentada por Hassler Whitney com a demonstração do seguinte teorema ${ }^{11}$.

Teorema 1 (de Whitney) Toda variedade diferenciável com D dimensões pode ser imersa no $\mathbb{R}^{2 \mathrm{D}}$ e mergulhada no $\mathbb{R}^{2 \mathrm{D}+1}$.

Contudo devemos frisar que, independente deste particular resultado, são vários os exemplos de variedades imersas em espaços Euclideanos não satisfazendo a estas condições, onde o mais conhecido talvez seja o das esferas D-dimensionais definidas no $\mathbb{R}^{\mathrm{D}+1}$. Tais exemplos servem para ilustrar um fato fundamental:

O teorema de Whitney não apresenta qualquer tipo de proibição em imergir variedades com D dimensões num $\mathbb{R}^{\mathrm{N}}$ onde $\mathrm{N}<2 \mathrm{D}$. Ele apenas diz que é sempre possível realizar tal processo em espaços Euclideanos com dimensões maiores ou iguais a 2D .

Respostas mais gerais e específicas ao mesmo questionamento foram dadas por John Nash e, uma delas, se resume no teorema a seguir [28].

Teorema 2 (de Nash) Qualquer variedade com $\mathrm{D}$ dimensões e métrica positiva de classe $C^{\mathrm{k}}$, onde $3 \leqslant \mathrm{k} \leqslant \infty$, possui um mergulho também isométrico de classe $C^{\mathrm{k}}$ no $\mathbb{R}^{\mathrm{D}(\mathrm{D}+1)(3 \mathrm{D}+11) / 2}$, especialmente em quaisquer dos seus abertos.

Deste modo, visto que espaços Riemannianos com D dimensões se enquadram como exemplos destas variedades, sempre podemos mergulhá-los, e logo imergí-los, isometricamente nos Euclideanos com dimensões maiores ou iguais a $D(D+1)(3 D+11) / 2$.

\footnotetext{
${ }^{10}$ Aliás no que segue, sempre que os símbolos $\|$ e $\perp$ estiverem presentes, eles referir-se-ão a elementos, respectivamente, tangentes e perpendiculares a algum ambiente em específico.

${ }^{11}$ Omitiremos aqui a demonstração deste teorema apenas por fugir do escopo do trabalho. Uma simples e excelente demonstração consta, por exemplo, em [27].
} 


\subsubsection{Algumas particularidades}

Com base em todas essas observações, devemos ilustrar algumas das características específicas associadas ao mergulho isométrico de uma variedade Riemanniana $\mathcal{M}$ num espaço Euclideano com $\mathrm{N}$ dimensões extras, úteis ao que segue nos próximos capítulos. Para isso tomaremos $q=$ $\left(q^{1}, \ldots, q^{\mathrm{D}}\right)$ como os parâmetros intrínsecos a $\mathcal{M}$ em algum dos seus abertos $\mathrm{U}$, onde por consequência $g_{\mu \nu}(q)$ são as componentes do seu tensor métrico ${ }^{12}$, e $x=\left(x^{1}, \ldots, x^{\mathrm{D}+\mathrm{N}}\right)$ como os parâmetros associados ao $\mathbb{R}^{\mathrm{D}+\mathrm{N}}$ no qual $\mathcal{M}$ se mergulha.

De acordo com o apresentado em páginas anteriores, o mergulho de $\mathcal{M}$ neste ambiente Euclideano permite fazer a decomposição específica

$$
T_{\mathrm{q}} \mathbb{R}^{\mathrm{D}+\mathrm{N}}=T_{\mathrm{q}} \mathcal{M} \oplus\left(T_{\mathrm{q}} \mathcal{M}\right)^{\perp}
$$

visto o produto interno definido no $\mathbb{R}^{\mathrm{D}+\mathrm{N}}$, sendo q um ponto de $\mathcal{M}$. Em particular, uma vez que espaços Euclideanos também se parametrizam por sistemas de coordenadas curvilíneas, essa mesma decomposição permite ao $\mathbb{R}^{\mathrm{D}+\mathrm{N}}$ ser parametrizado por $Q=(q, \mathcal{Q})$ em seu aberto $\mathrm{U}$, sendo $\mathcal{Q}=\left(\mathcal{Q}^{1}, \ldots, \mathcal{Q}^{\mathbb{N}}\right)$ os parâmetros "extrínsecos" a $\mathcal{M}_{U}{ }^{13}$ : ou seja, estes últimos referir-se-ão a $\mathcal{M}_{U}$ apenas quando forem identicamente nulos, permitindo identificar este ambiente como uma subvariedade do $\mathbb{R}^{\mathrm{D}+\mathrm{N}}$.

A consideração particular de um difeomorfismo $f: \mathbb{R}^{\mathrm{D}+\mathrm{N}} \rightarrow \mathbb{R}^{\mathrm{D}+\mathrm{N}}$ também permite observar que ambas parametrizações se relacionam, por exemplo, através de ${ }^{14}$

$$
Q^{j}=f^{j}(x)-c^{j}
$$

onde $c^{\mathrm{j}}$ são constantes em $\mathbb{R}$. Neste caso, para a subvariedade em questão, fica válido que $f^{\mathrm{A}}(x)=$ $c^{\mathrm{A}}$, realçando a interpretação de $\mathcal{M}_{U}$ como uma superfície do $\mathbb{R}^{\mathrm{D}+\mathrm{N}}$.

Com o efeito da forma métrica de qualquer variedade Riemanniana ser invariante, quaisquer sejam os parâmetros adotados [12], é notável que, ao longo de $\mathcal{M}_{\mathrm{U}}$,

$$
d s^{2}=\tilde{g}_{\mathrm{jk}}(Q) d Q^{\mathrm{j}} d Q^{\mathrm{k}}=\delta_{\mathrm{rs}} d x^{\mathrm{r}} d x^{\mathrm{s}},
$$

\footnotetext{
${ }^{12}$ Em respeito ao resultado de Nash, assumiremos que cada uma destas componentes é uma função de classe $C^{\mathrm{k}}$, onde $3 \leqslant \mathrm{k} \leqslant \infty$.

${ }^{13}$ No caso $\mathcal{M} \cup$ denota a restrição de $\mathcal{M}$ ao aberto $U$.

${ }^{14}$ Utilizamos esta expressão apenas por conveniência ao que propomos apresentar.
} 
onde $\tilde{g}_{\text {jk }}(Q)$ são as componentes do tensor métrico do $\mathbb{R}^{\mathrm{D}+\mathrm{N}}$ em termos das coordenadas curvilíneas. Deste modo, é possível estabelecer uma relação entre as duas métricas envoltas no ambiente Euclideano por

$$
\tilde{g}_{\mathrm{jk}}(Q)=\delta_{\mathrm{rs}} \frac{\partial x^{\mathrm{r}}}{\partial Q^{\mathrm{j}}} \frac{\partial x^{\mathrm{s}}}{\partial Q^{\mathrm{k}}}
$$

Através desta expressão em particular, observamos

$$
g_{\mu \nu}(q)=\tilde{g}_{\mu \nu}(q, \mathcal{Q}=0)=\left.\delta_{\mathrm{jk}} \frac{\partial x^{\mathrm{j}}}{\partial q^{\mu}} \frac{\partial x^{\mathrm{k}}}{\partial q^{\nu}}\right|_{\mathcal{Q}=0}
$$

como as componentes do tensor métrico induzido em $\mathcal{M}_{U}$ pelo ambiente Euclideano, coincidindo com o originalmente definido, e

$$
G_{\mathrm{AB}}(q)=\tilde{g}_{\mathrm{AB}}(q, \mathcal{Q}=0)=\left.\delta_{j \mathrm{k}} \frac{\partial x^{\mathrm{j}}}{\partial \mathcal{Q}^{\mathrm{A}}} \frac{\partial x^{\mathrm{k}}}{\partial \mathcal{Q}^{\mathrm{B}}}\right|_{\mathcal{Q}=0}
$$

referindo-se às componentes do tensor métrico induzido no espaço normal a $\mathcal{M}_{\mathrm{U}}$, em cada um dos pontos da subvariedade. Além disso, já que os parâmetros $\mathcal{Q}^{\mathrm{A}}$ não nulos se referem exclusivamente ao ambiente externo a $\mathcal{M}_{\mathrm{U}}$, necessariamente também deve ser válido na subvariedade que

$$
N_{\mu \mathrm{B}}(q)=\tilde{g}_{\mu \mathrm{B}}(q, \mathcal{Q}=0)=\left.\delta_{j \mathrm{k}} \frac{\partial x^{\mathrm{j}}}{\partial q^{\mu}} \frac{\partial x^{\mathrm{k}}}{\partial \mathcal{Q}^{\mathrm{B}}}\right|_{\mathcal{Q}=0}=0
$$

Frente ao caráter difeomórfico de $f$, é imediato que os vetores $\partial x^{\mathrm{j}} / \partial Q^{\mathrm{k}}$ são linearmente independentes ${ }^{15}$. Logo a representação $\left(\tilde{g}_{\text {jk }}\right)$ é uma matriz de Gram positiva, portanto invertível ${ }^{16}$. No caso, temos que

$$
\tilde{g}^{\mathrm{jk}}=\delta^{\mathrm{rs}} \frac{\partial Q^{\mathrm{j}}}{\partial x^{\mathrm{r}}} \frac{\partial Q^{\mathrm{k}}}{\partial x^{\mathrm{s}}}
$$

onde em $\mathcal{M}_{U}$ vale particularmente

$$
g^{\mu \nu}=\left.\delta^{\mathrm{rs}} \frac{\partial q^{\mu}}{\partial x^{\mathrm{r}}} \frac{\partial q^{\nu}}{\partial x^{\mathrm{s}}}\right|_{\mathcal{Q}=0} \quad, G^{\mathrm{AB}}=\left.\delta^{\mathrm{jk}} \frac{\partial \mathcal{Q}^{\mathrm{A}}}{\partial x^{\mathrm{j}}} \frac{\partial \mathcal{Q}^{\mathrm{B}}}{\partial x^{\mathrm{k}}}\right|_{\mathcal{Q}=0} \quad \text { e } N^{\mu \mathrm{B}}=\left.\delta^{\mathrm{jk}} \frac{\partial q^{\mu}}{\partial x^{\mathrm{j}}} \frac{\partial \mathcal{Q}^{\mathrm{B}}}{\partial x^{\mathrm{k}}}\right|_{\mathcal{Q}=0}=0
$$

\footnotetext{
${ }^{15}$ Vide as considerações iniciais deste capítulo.

${ }^{16}$ Uma matriz de Gram $M$ se define por $M_{\mathrm{jk}}=\left\langle v_{\mathrm{j}}, v_{\mathrm{k}}\right\rangle$, onde $\left\{v_{1}, \ldots, v_{\mathrm{m}}\right\}$ é um conjunto de vetores pertencentes a um espaço vetorial $\mathbb{E}$ com dimensão finita. No caso, se prova que toda matriz de Gram é

(i) não negativa, e

(ii) positiva se, e somente se, $\left\{v_{1}, \ldots, v_{\mathrm{m}}\right\}$ for constituída tão somente por vetores linearmente independentes.
}

Para maiores detalhes sobre este assunto, remetemos o leitor a [29], especialmente à página 213. 


\subsection{Características associadas às imersões isométricas}

Antes de encerrarmos este capítulo, devemos fazer algumas observações adicionais referentes ao processo de imersão isométrica de uma variedade $\mathcal{M}$ com $\mathrm{D}$ dimensões em outra $\mathcal{N}$, com $\mathrm{N}$ dimensões extras. Uma destas observações se associa necessariamente à conexão Riemanniana ${ }^{17}$ da última variedade, denotada aqui por $\bar{\nabla}$ : tomando $X$ e $Y$ como dois campos locais formados por vetores em $\mathcal{M}$, assim como $\bar{X}$ e $\bar{Y}$ são as suas respectivas extensões também locais sobre $\mathcal{N}$, podemos definir

$$
\nabla_{X} Y=\left(\bar{\nabla}_{\bar{X}} \bar{Y}\right)^{\|}
$$

como a conexão Riemanniana relativa à métrica induzida em $\mathcal{M}$ [23]. Trata-se de uma relação de considerável importância, uma vez que dela é possível construir um campo local em $\mathcal{N}$, necessariamente normal a $\mathcal{M}$, através da aplicação não apenas bilinear, mas também simétrica $B: \mathcal{X}(\mathrm{U}) \times \mathcal{X}(\mathrm{U}) \rightarrow \mathcal{X}(\mathrm{U})^{\perp}$ dada por $^{18}$

$$
B(X, Y)=\bar{\nabla}_{\bar{X}} \bar{Y}-\nabla_{X} Y
$$

onde $\mathcal{X}(\mathrm{U})$ se refere a um conjunto dos campos de vetores de classe $C^{\infty}$ em $\mathrm{U} \subset \mathcal{M}$.

Uma das utilidades desta aplicação se associa ao fato de, com ela, podermos definir outra $B_{\eta}: T_{\mathbf{q}} \mathcal{M} \times T_{\mathbf{q}} \mathcal{M} \rightarrow \mathbb{R}$, para um vetor $\eta$ que pertence ao $\left(T_{\mathbf{q}} \mathcal{M}\right)^{\perp}$, através do produto interno

$$
B_{\eta}(x, y)=\langle B(x, y), \eta\rangle, \operatorname{com} x, y \in T_{\mathbf{q}} \mathcal{M}
$$

sendo possível definir a bem conhecida segunda forma fundamental $I I_{\eta}(x)$ para o caso de uma imersão $\varphi: \mathcal{M} \rightarrow \mathcal{N}$ num ponto q em específico, e segundo o mesmo $\eta$ supracitado. Explicitamente trata-se de uma forma quadrátrica definida em $T_{\mathrm{q}} \mathcal{M}$, dada por

$$
I I_{\eta}(x)=B_{\eta}(x, x)
$$

Algo bem interessante e associado diretamente à última expressão se refere, por exemplo, ao caráter das geodésicas que eventualmente figuram em $\mathcal{M}$. No caso, se toda geodésica $\gamma$ definida

\footnotetext{
${ }^{17} \mathrm{Ou}$ seja, uma conexão afim não apenas simétrica, mas compatível com a métrica Riemanniana. Uma apresentação simples, didática e indubitavelmente rigorosa sobre estas definições, assim como aos teoremas a elas associados, se encontra em [23], mais especificamente em seu Capítulo 2.

${ }^{18}$ Aliás, por esta bilinearidade e sob a adoção de um sistema de coordenadas arbitrário, é demonstrável que $B(X, Y)(\mathbf{q})$ depende tão somente dos valores $X(\mathbf{q})$ e $Y(\mathbf{q})$.
} 
na subvariedade $\mathcal{M}$, e que passe por algum dos seus pontos q, também se comportar como uma geodésica em $\mathcal{N}$, a segunda forma fundamental $I I_{\eta}$ será identicamente nula neste mesmo ponto. Quando isso ocorre, dizemos que $\varphi$ é uma imersão geodésica ao menos em q, sendo totalmente geodésica apenas se o caráter nulo de $I I_{\eta}$ se estender aos demais pontos de $\mathcal{M}$.

Apenas para completar esta seção, devemos notar que da mesma $I I_{\eta}$ podemos construir outra aplicação linear, necessariamente autoadjunta, $S_{\eta}: T_{\mathrm{q}} \mathcal{M} \rightarrow T_{\mathrm{q}} \mathcal{M}$ através de

$$
\left\langle S_{\eta}(x), y\right\rangle=B_{\eta}(x, y)=\langle B(x, y), \eta\rangle, \operatorname{com} x, y \in T_{\mathbf{q}} \mathcal{M}
$$

Trata-se de uma aplicação que permite-nos verificar outro fato referente às imersões que, embora não seja tão forte quanto o anterior, também tem a sua valia.

Definição 1 Dizemos que $\varphi: \mathcal{M} \rightarrow \mathcal{N}$ é uma imersão mínima num ponto q pertencente a $\mathcal{M}$, sobre o qual definimos $\eta$ em $\left(T_{\mathbf{q}} \mathcal{M}\right)^{\perp}$, se o traço de $S_{\eta}$ for nulo.

Deste modo, ao escolhermos um referencial cuja base é o conjunto $\left\{\mathrm{e}^{1}, \ldots, \mathrm{e}^{\mathrm{N}}\right\}$ de vetores ortonormais do $\mathcal{X}(\mathrm{U})^{\perp}$, sendo $\mathrm{U}$ uma vizinhança de q e na qual $\varphi$ é considerada uma imersão, podemos escrever, neste mesmo ponto, que

$$
B(x, y)=B_{\mathrm{A}}(x, y) \mathrm{e}^{\mathrm{A}},
$$

onde $B_{\mathrm{A}}=B_{\mathrm{e}^{\mathrm{A}}}$. Neste caso, o vetor de curvatura média $\mathrm{H}$, normal à então subvariedade, se expressa por

$$
\mathrm{H}=\frac{1}{\mathrm{D}} \operatorname{Tr}\left(S_{\mathrm{A}}\right) \mathrm{e}^{\mathrm{A}},
$$

$\operatorname{com} S_{\mathrm{A}}=S_{\mathrm{e}^{\mathrm{A}}}$, e independe do referencial adotado. Aliás, por esta expressão e segundo a definição acima, $\varphi$ tratar-se-á de uma imersão mínima se, e somente se, $\mathrm{H}(\mathbf{q})=0$ em todos os pontos q de $\mathcal{M}$

De acordo com [23], o motivo desta nomenclatura é devido, tão somente, ao fato desta imersão em específico ser capaz de minimizar os volumes que se originam pela métrica induzida na então subvariedade, da mesma maneira que as geodésicas tornam mínimo o comprimento dos arcos por elas descritos.

Apesar da segunda forma fundamental ser definida em termos de derivadas covariantes de campos vetoriais pertencentes ao $T_{\mathbf{q}} \mathcal{M}$, também podemos usá-la na determinação de derivadas 
covariantes em campos normais a $\mathcal{M}$, conforme se prova no lema abaixo [30].

Lema 1 (Equação de Weingarten) Supondo que $x$ e y pertencem ao $T_{\mathbf{q}} \mathcal{M}$, assim como $\eta$ pertence $a\left(T_{\mathbf{q}} \mathcal{M}\right)^{\perp}$, ao tomarmos as respectivas extensões locais $X, Y$ e $N$ destes mesmos elementos em $\mathcal{N}$, vale que

$$
\left\langle\bar{\nabla}_{X} N, Y\right\rangle=-\langle N, B(X, Y)\rangle
$$

Deste lema em particular se demonstra, por exemplo e com o uso de $(2.12)$, que $S_{\eta}(x)=-\left(\bar{\nabla}_{x} N\right)^{\|}$. Basta notar que

$$
\left\langle\bar{\nabla}_{x} N, y\right\rangle=\left\langle\bar{\nabla}_{X} N, Y\right\rangle=-\langle N, B(X, Y)\rangle=-\left\langle S_{\eta}(x), y\right\rangle .
$$




\section{Capítulo 3}

\section{O problema da partícula}

\subsection{Considerações Lagrangianas}

Com base no resultado de Nash que variedades Riemannianas são mergulháveis em espaços Euclideanos, e portanto podem ser consideradas como superfícies regulares desde que condições dimensionais específicas sejam satisfeitas, usaremos este fato para a descrição clássica de um sistema físico num espaço Riemanniano $\mathcal{M}$ qualquer ${ }^{1}$ que admite ser parametrizado por um único sistema de coordenadas curvilíneas. Deste modo, e face aos objetivos do presente trabalho, definiremos nosso sistema físico por uma única partícula massiva, num movimento não relativístico vinculado a uma subvariedade $\mathcal{M}$ com $\mathrm{D}$ dimensões no $\mathbb{R}^{\mathrm{D}+\mathrm{N}}$, adicionalmente submetida a um potencial com dependência exclusiva das coordenadas do sistema.

De acordo com os comentários do capítulo anterior, a parametrização do espaço Euclideano por um sistema de coordenadas cartesianas $x=\left(x^{1}, \ldots, x^{\mathrm{D}+\mathrm{N}}\right)$ permite descrever a subvariedade $\mathcal{M}$ através das $\mathrm{N}$ equações

$$
f^{\mathrm{A}}(x)=0
$$

sendo $f^{\prime A}: \mathbb{R}^{\mathrm{D}+\mathrm{N}} \rightarrow \mathbb{R}$ sobrejetoras ${ }^{2}$. Com efeito, a descrição clássica deste sistema físico se faz por meio da Lagrangiana $L: T \mathbb{R}^{\mathrm{D}+2 \mathrm{~N}} \rightarrow \mathbb{R}$ dada por

$$
L(x, \lambda ; \dot{x}, \dot{\lambda})=\frac{m}{2} \delta_{\mathrm{jk}} \dot{x}^{\mathrm{j}} \dot{x}^{\mathrm{k}}-V(x)-\lambda^{\mathrm{A}} f_{\mathrm{A}}(x),
$$

\footnotetext{
${ }^{1}$ Cabe mencionar que o sistema físico pode estar definido apenas numa das vizinhanças de $\mathcal{M}$, ou até mesmo num dos seus abertos U. Apesar desta observação, denotaremos este ambiente, embora restrito, também por $\mathcal{M}$ de agora em diante, salvo menção ao contrário, não apenas por uma questão de simplicidade notacional, mas essencialmente devido ao fato de que abertos em $\mathcal{M}$ também figuram como variedades Riemannianas.

${ }^{2} \mathrm{~A}$ particular escolha por (3.1) segue da observação de que quaisquer superfícies regulares dadas por (2.2) são assim descritas por meio de uma transformação $f(x)-c \rightarrow f(x)$.
} 
onde $\lambda^{\mathrm{A}}$ são os multiplicadores de Lagrange utilizados na restrição do movimento da partícula sobre a subvariedade, também considerados como variáveis do problema assim como $\dot{\lambda}^{\mathrm{A}}$.

\subsection{O formalismo Hamiltoniano}

Conforme consta na literatura, a transição do formalismo Lagrangiano para o Hamiltoniano ocorre através de uma transformação de Legendre em L. Explicitamente, trata-se da substituição das velocidades pelos momentos canonicamente conjugados às variáveis de posição, supostos independentes [31]. No caso da Lagrangiana (3.2), os momentos conjugados a $x$ e $\lambda$ são dados respectivamente por

$$
P_{j}=\frac{\partial L}{\partial \dot{x}^{\mathrm{j}}}=m \delta_{j \mathrm{k}} \dot{x}^{\mathrm{k}} \text { e } \pi_{\mathrm{A}}=\frac{\partial L}{\partial \dot{\lambda}^{\mathrm{A}}}=0
$$

de onde seguem duas observações.

A primeira delas decorre da expressão de $P_{\mathrm{j}}$, visto a possibilidade de expressar cada $\dot{x}^{\mathrm{j}} \mathrm{em}$ termos das demais variáveis. Ou seja,

$$
\dot{x}^{\mathrm{j}}=v^{\mathrm{j}}(x, \lambda ; P, \pi)=\frac{1}{m} \delta^{\mathrm{jk}} P_{\mathrm{k}} .
$$

Já a segunda observação se respauda no fato da expressão para $\pi_{\mathrm{A}}$ trazê-los como identicamente nulos, quaisquer sejam os valores assumidos pelas demais variáveis, uma vez que a Lagrangiana independe de $\dot{\lambda}^{\mathrm{A}}$. Por consequência, e de acordo com o teorema da função inversa [32], não é possível expressar ${ }^{3}$ as velocidades $\dot{\lambda}^{\mathrm{A}}$ do sistema físico em termos das demais variáveis, em especial de $\pi_{\mathrm{A}}$. Desta maneira, observamos a presença dos $\mathrm{N}$ primeiros vínculos deste sistema, os quais são expressos por

$$
\Phi_{\mathrm{A}}(x, \lambda ; P, \pi)=\pi_{\mathrm{A}}=0
$$

Em particular, face à sobrejetividade de $\Phi_{\mathrm{A}}^{\prime}: T^{*} \mathbb{R}^{\mathrm{D}+2 \mathrm{~N}} \rightarrow \mathbb{R}$, notamos que (3.5) define uma parte das componentes de uma superfície regular no espaço de fase, na qual o sistema físico reside. Logo o momento $\pi$ é nulo apenas ao longo desta e não em todo o espaço de fase.

Devido a estas características e em respeito ao procedimento de Dirac [24], as três últimas equações permitem definir $H_{P}: T^{*} \mathbb{R}^{\mathrm{D}+2 \mathrm{~N}} \rightarrow \mathbb{R}$ como a Hamiltoniana primária no ambiente Euclideano através de

$$
H_{P}=H+\lambda^{\mathrm{A}} f_{\mathrm{A}}(x)+\xi^{\mathrm{A}} \pi_{\mathrm{A}},
$$

\footnotetext{
${ }^{3}$ Ao menos por enquanto, e de acordo com as atuais considerações.
} 
onde $H: T^{*} \mathbb{R}^{\mathrm{D}+2 \mathrm{~N}} \rightarrow \mathbb{R}$ é dada por

$$
H=\frac{1}{2 m} \delta^{\mathrm{jk}} P_{\mathrm{j}} P_{\mathrm{k}}+V(x), \text { e }
$$

$\xi^{\text {A }}$ se definem como multiplicadores de Lagrange utilizados não apenas para restringir a dinâmica da partícula ao fibrado cotangente mergulhado ${ }^{4}$ em $T^{*} \mathbb{R}^{\mathrm{D}+2 \mathrm{~N}}$, mas também para estabelecer uma relação isomórfica entre $T \mathbb{R}^{\mathrm{D}+2 \mathrm{~N}}$ e o próprio $T^{*} \mathbb{R}^{\mathrm{D}+2 \mathrm{~N}}$ perante à transformação de Legendre ${ }^{5}[3]$.

\subsection{As equações de consistência}

Uma vez que (3.5) se refere às componentes de uma superfície regular onde o movimento da partícula ocorre, devemos impor adicionalmente a todos os vínculos primários que

$$
\dot{\Phi}_{\mathrm{A}}=\left\{\Phi_{\mathrm{A}}, H_{P}\right\}=0,
$$

afinal cada vínculo, e portanto a superfície por eles gerada, figura como uma constante de movimento no espaço de fase. O desenvolvimento desta particular relação, e o uso de (3.5) e (3.6), mostra-nos que

$$
\dot{\Phi}_{\mathrm{A}}=\left\{\pi_{\mathrm{A}}, \lambda^{\mathrm{B}}\right\} f_{\mathrm{B}}(x)=-\delta_{\mathrm{A}}^{\mathrm{B}} f_{\mathrm{B}}(x)=-f_{\mathrm{A}}(x)=0 .
$$

Assim, por tratar-se de uma expressão que independe tanto dos multiplicadores $\xi^{\mathrm{A}}$ como dos vínculos primários, vemos a submissão do sistema físico também aos vínculos

$$
\Phi_{\mathrm{N}+\mathrm{A}}(x, \lambda ; P, \pi)=f_{\mathrm{A}}(x)=0
$$

também responsáveis pela composição da superfície onde todo o movimento ocorre. Em verdade tratam-se de vínculos já esperados, dado que as mesmas expressões definem a subvariedade Riemanniana (3.1).

Particularmente, a aparição dos vínculos (3.9) frente a esta formulação permite interpretar $H$ como a Hamiltoniana do sistema físico, visto tratar-se da forma assumida por $H_{P}$ ao longo da superfície de movimentação contida no $T^{*} \mathbb{R}^{\mathrm{D}+2 \mathrm{~N}}$, em adicional acordo de também ser a expressão para a energia segundo o formalismo Lagrangiano [33].

\footnotetext{
${ }^{4}$ Isso se deve ao fato de $T^{*} \mathcal{M}$ ser caracterizado como uma variedade [23], logo podendo ser mergulhado em outra dimensionalmente maior. No caso, este comportamento de subvariedade é revelado pelas expressões do vínculos $\Phi$ explicitamente.

${ }^{5}$ Devemos notar que este isomorfismo fica estabelecido ao tomarmos $\xi^{\mathrm{A}}=\dot{\lambda}^{\mathrm{A}}$.
} 
Da imposição de condições análogas de consistência sobre as componentes $\Phi_{\mathrm{N}+\mathrm{A}}$, nota-se que

$$
\dot{\Phi}_{\mathrm{N}+\mathrm{A}}=\left\{\Phi_{\mathrm{N}+\mathrm{A}}, H_{P}\right\}=0 \Rightarrow \frac{1}{m} P^{\mathrm{j}}\left\{f_{\mathrm{A}}(x), P_{\mathrm{j}}\right\}=\frac{1}{m} P^{\mathrm{j}} \frac{\partial f_{\mathrm{A}}}{\partial x^{\mathrm{j}}}=0
$$

de onde é perceptível a submissão do sistema físico também aos vínculos expressos por

$$
\Phi_{2 \mathrm{~N}+\mathrm{A}}(x, \lambda ; P, \pi)=m \dot{\Phi}_{\mathrm{N}+\mathrm{A}}=P^{\mathrm{j}} \frac{\partial f_{\mathrm{A}}}{\partial x^{\mathrm{j}}}=0
$$

dado independência funcional destes em relação aos anteriores, especialmente dos multiplicadores $\xi^{\mathrm{A}}$. Em particular, observamos da última expressão, com o efeito de $\partial f_{\mathrm{A}} / \partial x$ serem normais à superfície definida em (3.1), que o momento $P$ deve necessariamente pertencer ao plano tangente da mesma.

Dando sequência ao mesmo raciocínio, temos

$$
\begin{aligned}
\dot{\Phi}_{2 \mathrm{~N}+\mathrm{A}} & =\left\{\Phi_{2 \mathrm{~N}+\mathrm{A}}, H_{P}\right\}=0 \\
& =\frac{1}{m} P^{\mathrm{j}} P^{\mathrm{k}}\left\{\frac{\partial f_{\mathrm{A}}}{\partial x^{\mathrm{j}}}, P_{\mathrm{k}}\right\}+\frac{\partial f_{\mathrm{A}}}{\partial x^{\mathrm{j}}}\left\{P^{\mathrm{j}}, V(x)\right\}+\lambda^{\mathrm{B}} \frac{\partial f_{\mathrm{A}}}{\partial x^{\mathrm{j}}}\left\{P_{\mathrm{j}}, f_{\mathrm{B}}(x)\right\}=0,
\end{aligned}
$$

originando os vínculos

$$
\Phi_{3 \mathrm{~N}+\mathrm{A}}(x, \lambda ; P, \pi)=\dot{\Phi}_{2 \mathrm{~N}+\mathrm{A}}=\frac{1}{m} P^{\mathrm{j}} P^{\mathrm{k}} \frac{\partial^{2} f_{\mathrm{A}}}{\partial x^{\mathrm{j}} \partial x^{\mathrm{k}}}-\delta^{\mathrm{jk}} \frac{\partial f_{\mathrm{A}}}{\partial x^{\mathrm{j}}}\left(\frac{\partial V}{\partial x^{\mathrm{k}}}+\lambda^{\mathrm{B}} \frac{\partial f_{\mathrm{B}}}{\partial x^{\mathrm{k}}}\right)=0
$$

independentes não apenas dos anteriores, mas também dos multiplicadores suprarreferidos, e de onde vemos que

$$
\begin{aligned}
\dot{\Phi}_{3 \mathrm{~N}+\mathrm{A}} & =\left\{\Phi_{3 \mathrm{~N}+\mathrm{A}}, H_{P}\right\}=0 \\
& =\frac{1}{m^{2}} P^{\mathrm{j}} P^{\mathrm{k}} P^{1}\left\{\frac{\partial^{2} f_{\mathrm{A}}}{\partial x^{\mathrm{j}} \partial x^{\mathrm{k}}}, P_{1}\right\}+\frac{2}{m} \delta^{\mathrm{kl} l} P^{\mathrm{j}} \frac{\partial^{2} f_{\mathrm{A}}}{\partial x^{\mathrm{j}} \partial x^{\mathrm{k}}}\left\{P_{1}, V(x)\right\} \\
& +\frac{2}{m} \delta^{\mathrm{kl}} P^{\mathrm{j}} \lambda^{\mathrm{B}} \frac{\partial^{2} f_{\mathrm{A}}}{\partial x^{\mathrm{j}} \partial x^{\mathrm{k}}}\left\{P_{1}, f_{\mathrm{B}}(x)\right\}-\frac{1}{m} \delta^{\mathrm{jk}} P^{1}\left\{\frac{\partial f_{\mathrm{A}}}{\partial x^{\mathrm{j}}} \frac{\partial V}{\partial x^{\mathrm{k}}}, P_{1}\right\} \\
& -\frac{1}{m} \delta^{\mathrm{jk}} \lambda^{\mathrm{B}} P^{1}\left\{\frac{\partial f_{\mathrm{A}}}{\partial x^{\mathrm{j}}} \frac{\partial f_{\mathrm{B}}}{\partial x^{\mathrm{k}}}, P_{1}\right\}+\delta^{\mathrm{jk}} \xi^{\mathrm{C}} \frac{\partial f_{\mathrm{A}}}{\partial x^{\mathrm{j}}} \frac{\partial f_{\mathrm{B}}}{\partial x^{\mathrm{k}}}\left\{\lambda^{\mathrm{B}}, \pi_{\mathrm{C}}\right\}=0 .
\end{aligned}
$$

Devido à presença dos multiplicadores $\xi^{\mathrm{A}}$ nesta igualdade, nenhum vínculo adicional surge por este procedimento, face a eventual possibilidade de resolução destes multiplicadores em termos das 
demais variáveis envolvidas. Aliás, de acordo com a mesma igualdade, tem-se

$$
\begin{aligned}
\delta^{j \mathrm{k}} \xi^{\mathrm{B}} \frac{\partial f_{\mathrm{A}}}{\partial x^{\mathrm{j}}} \frac{\partial f_{\mathrm{B}}}{\partial x^{\mathrm{k}}} & =\delta^{\mathrm{jk}} \xi^{\mathrm{C}}\left\{\lambda^{\mathrm{B}}, \pi_{\mathrm{C}}\right\} \frac{\partial f_{\mathrm{A}}}{\partial x^{\mathrm{j}}} \frac{\partial f_{\mathrm{B}}}{\partial x^{\mathrm{k}}} \\
& =-\frac{1}{m^{2}} P^{\mathrm{j}} P^{\mathrm{k}} P^{1} \frac{\partial^{3} f_{\mathrm{A}}}{\partial x^{\mathrm{j}} \partial x^{\mathrm{k}} \partial x^{1}}+\frac{2}{m} \delta^{\mathrm{k} \mathrm{l}} P^{\mathrm{j}} \frac{\partial^{2} f_{\mathrm{A}}}{\partial x^{\mathrm{j}} \partial x^{\mathrm{k}}}\left(\frac{\partial V}{\partial x^{1}}+\lambda^{\mathrm{B}} \frac{\partial f_{\mathrm{B}}}{\partial x^{1}}\right) \\
& +\frac{1}{m} \delta^{j \mathrm{k}} P^{1} \frac{\partial}{\partial x^{1}}\left(\frac{\partial f_{\mathrm{A}}}{\partial x^{\mathrm{j}}} \frac{\partial V}{\partial x^{\mathrm{k}}}\right)+\frac{1}{m} \delta^{\mathrm{jk}} \lambda^{\mathrm{B}} P^{1} \frac{\partial}{\partial x^{1}}\left(\frac{\partial f_{\mathrm{A}}}{\partial x^{\mathrm{j}}} \frac{\partial f_{\mathrm{B}}}{\partial x^{\mathrm{k}}}\right) .
\end{aligned}
$$

\subsubsection{Sobre o conjunto de vínculos}

Tomemos $A$ como uma matriz quadrada de ordem $\mathrm{N}$ dada por

$$
A_{\mathrm{AB}}=\delta^{\mathrm{jk}} \frac{\partial f_{\mathrm{A}}}{\partial x^{\mathrm{j}}} \frac{\partial f_{\mathrm{B}}}{\partial x^{\mathrm{k}}}
$$

Frente à caracterização de $\mathcal{M}$ como superfície regular trazer necessariamente os $\mathrm{N}$ vetores $\partial f_{\mathrm{A}} / \partial x$ como linearmente independentes, se observa que $A$ é uma matriz de Gram positiva, assim como as mencionadas no capítulo anterior. Deste modo, existe a inversa $A^{-1}$ e a equação (3.13) se torna ${ }^{6}$

$$
\begin{aligned}
\xi^{\mathrm{A}} & =\bar{\xi}^{\mathrm{A}}(x, \lambda ; P, \pi)=-\frac{1}{m^{2}} P^{\mathrm{j}} P^{\mathrm{k}} P^{\mathrm{l}} A^{\mathrm{AB}} \frac{\partial^{3} f_{\mathrm{B}}}{\partial x^{\mathrm{j}} \partial x^{\mathrm{k}} \partial x^{1}}+\frac{2}{m} \delta^{\mathrm{k} \mathrm{l}} P^{\mathrm{j}} A^{\mathrm{AB}} \frac{\partial^{2} f_{\mathrm{B}}}{\partial x^{\mathrm{j}} \partial x^{\mathrm{k}}}\left(\frac{\partial V}{\partial x^{1}}+\lambda^{\mathrm{C}} \frac{\partial f_{\mathrm{C}}}{\partial x^{\mathrm{l}}}\right) \\
& +\frac{1}{m} \delta^{\mathrm{jk}} P^{\mathrm{l}} A^{\mathrm{AB}} \frac{\partial}{\partial x^{1}}\left(\frac{\partial f_{\mathrm{B}}}{\partial x^{\mathrm{j}}} \frac{\partial V}{\partial x^{\mathrm{k}}}\right)+\frac{1}{m} \delta^{\mathrm{jk}} \lambda^{\mathrm{C}} P^{1} A^{\mathrm{AB}} \frac{\partial}{\partial x^{1}}\left(\frac{\partial f_{\mathrm{B}}}{\partial x^{\mathrm{j}}} \frac{\partial f_{\mathrm{C}}}{\partial x^{\mathrm{k}}}\right),
\end{aligned}
$$

expressando todos os multiplicadores de Lagrange $\xi^{\mathrm{A}}$ em termos das demais variáveis. Pelo mesmo raciocínio, o uso de (3.11) traz

$$
\delta^{\mathrm{jk}} \frac{\partial f_{\mathrm{A}}}{\partial x^{\mathrm{j}}}\left(\frac{\partial V}{\partial x^{\mathrm{k}}}+\lambda^{\mathrm{B}} \frac{\partial f_{\mathrm{B}}}{\partial x^{\mathrm{k}}}\right)=\frac{1}{m} P^{\mathrm{j}} P^{\mathrm{k}} \frac{\partial^{2} f_{\mathrm{A}}}{\partial x^{\mathrm{j}} \partial x^{\mathrm{k}}}
$$

de onde segue a possibilidade de exprimir cada $\lambda^{\mathrm{A}}$ em função das variáveis $x$ e $P$, também visto a não singularidade de $A$. Especificamente, através de simples manobras algébricas, obtemos

$$
\lambda^{\mathrm{A}}=\bar{\lambda}^{\mathrm{A}}(x, P)=A^{\mathrm{AB}}\left(\frac{1}{m} P^{\mathrm{j}} P^{\mathrm{k}} \frac{\partial^{2} f_{\mathrm{B}}}{\partial x^{\mathrm{j}} \partial x^{\mathrm{k}}}-\delta^{\mathrm{jk}} \frac{\partial f_{\mathrm{B}}}{\partial x^{\mathrm{j}}} \frac{\partial V}{\partial x^{\mathrm{k}}}\right),
$$

o que reforça a interpretação de $\lambda^{\mathrm{A}}$ como multiplicadores de Lagrange, conforme preconiza o formalismo Lagrangiano. Com efeito, os multiplicadores $\xi^{\mathrm{A}}$ se expressam em termos das mesmas

\footnotetext{
${ }^{6}$ Aqui, $A^{\mathrm{AB}} A_{\mathrm{BC}}=\delta_{\mathrm{C}}^{\mathrm{A}}$.
} 
variáveis, uma vez que

$$
\xi^{\mathrm{A}}=\bar{\xi}^{\mathrm{A}}(x, \bar{\lambda}(x, P) ; P, \bar{\pi}(x, P))=\overline{\bar{\xi}}^{\mathrm{A}}(x, P)
$$

visto a definição de $\pi$ feita em (3.5). Ou seja $\lambda, \pi: T^{*} \mathbb{R}^{\mathrm{D}+\mathrm{N}} \rightarrow \mathbb{R}$ ficam restritas à subvariedade $T^{*} \mathcal{M} \subset T^{*} \mathbb{R}^{\mathrm{D}+\mathrm{N}}$, se descrevendo por $z=(x, P)$ com o advento da presença dos vínculos

$$
\Phi_{\mathrm{A}}^{*}(x, P)=f_{\mathrm{A}}(x)=0, \Phi_{\mathrm{N}+\mathrm{A}}^{*}(x, P)=P^{\mathrm{j}} \frac{\partial f_{\mathrm{A}}}{\partial x^{\mathrm{j}}}=0,
$$

nitidamente de segunda classe ${ }^{7}$, os únicos necessários à definição do mesmo fibrado cotangente $T^{*} \mathcal{M}$. Por se dizer, perante esta redução de vínculos, as equações de movimento associadas ao sistema físico tornam-se

$$
\dot{z}=\left.\{z, H\}_{D\left(\Phi^{*}\right)}\right|_{\Phi^{*}=0}
$$

\subsection{Algumas relações clássicas}

Através de (3.16) em particular, é possível observar que

$$
\begin{aligned}
& \left\{\Phi_{\mathrm{A}}^{*}, \Phi_{\mathrm{B}}^{*}\right\}=\left\{f_{\mathrm{A}}(x), f_{\mathrm{B}}(x)\right\}=0, \\
& \left\{\Phi_{\mathrm{A}}^{*}, \Phi_{\mathrm{N}+\mathrm{B}}^{*}\right\}=\delta^{\mathrm{jk}} \frac{\partial f_{\mathrm{B}}}{\partial x^{\mathrm{j}}}\left\{f_{\mathrm{A}}(x), P_{\mathrm{k}}\right\}=\delta^{\mathrm{jk}} \frac{\partial f_{\mathrm{A}}}{\partial x^{\mathrm{k}}} \frac{\partial f_{\mathrm{B}}}{\partial x^{\mathrm{j}}}, \\
& \left\{\Phi_{\mathrm{N}+\mathrm{A}}^{*}, \Phi_{\mathrm{N}+\mathrm{B}}^{*}\right\}=P^{\mathrm{j}} \frac{\partial f_{\mathrm{B}}}{\partial x^{\mathrm{k}}} \delta^{\mathrm{kb}}\left\{\frac{\partial f_{\mathrm{A}}}{\partial x^{\mathrm{j}}}, P_{\mathrm{b}}\right\}+P^{\mathrm{k}} \frac{\partial f_{\mathrm{A}}}{\partial x^{\mathrm{j}}} \delta^{\mathrm{ja}}\left\{P_{\mathrm{a}}, \frac{\partial f_{\mathrm{B}}}{\partial x^{\mathrm{k}}}\right\} \\
& =P^{\mathrm{j}} \delta^{\mathrm{kl}}\left(\frac{\partial^{2} f_{\mathrm{A}}}{\partial x^{\mathrm{j}} \partial x^{1}} \frac{\partial f_{\mathrm{B}}}{\partial x^{\mathrm{k}}}-\frac{\partial f_{\mathrm{A}}}{\partial x^{\mathrm{k}}} \frac{\partial^{2} f_{\mathrm{B}}}{\partial x^{\mathrm{j}} \partial x^{1}}\right) \text {. }
\end{aligned}
$$

Sob a adoção de $\Theta$ por uma matriz quadrada de ordem 2N, com elementos dados por $\Theta_{\mathrm{ab}}=$ $\left\{\Phi_{\mathrm{a}}^{*}, \Phi_{\mathrm{b}}^{*}\right\}$, notamos que

$$
\Theta=\left(\begin{array}{cc}
0 & A \\
-A & B
\end{array}\right),
$$

\footnotetext{
${ }^{7}$ Recomendamos a leitura do Apêndice A para uma breve exposição de alguns conceitos úteis ao que segue. Para uma discussão mais detalhada, sugerimos [7].
} 
onde $A$ e $B$ também são duas matrizes quadradas, porém de ordem $\mathrm{N}$, definidas por

$$
A_{\mathrm{AB}}=\left\{\Phi_{\mathrm{A}}^{*}, \Phi_{\mathrm{N}+\mathrm{B}}^{*}\right\} \quad, \quad B_{\mathrm{AB}}=\left\{\Phi_{\mathrm{N}+\mathrm{A}}^{*}, \Phi_{\mathrm{N}+\mathrm{B}}^{*}\right\} .
$$

Desta maneira, perante a não singularidade de $\Theta$ resumida em

$$
\Theta^{-1}=\left(\begin{array}{cc}
A^{-1} B A^{-1} & -A^{-1} \\
A^{-1} & 0
\end{array}\right)
$$

todos os possíveis parênteses de Dirac entre as variáveis $x$ e $P$ ficam expressos através de

$$
\begin{aligned}
\left\{x^{\mathrm{j}}, x^{\mathrm{k}}\right\}_{D\left(\Phi^{*}\right)} & =\left\{x^{\mathrm{j}}, x^{\mathrm{k}}\right\}=0 \\
\left\{x^{\mathrm{j}}, P_{\mathrm{k}}\right\}_{D\left(\Phi^{*}\right)} & =\left\{x^{\mathrm{j}}, P_{\mathrm{k}}\right\}-\delta^{\mathrm{ab}} \frac{\partial f_{\mathrm{A}}}{\partial x^{\mathrm{a}}}\left\{x^{\mathrm{j}}, P_{\mathrm{b}}\right\} A^{\mathrm{AB}}\left\{f_{\mathrm{B}}(x), P_{\mathrm{k}}\right\} \\
& =\delta_{\mathrm{k}}^{\mathrm{j}}-\delta^{\mathrm{j}} A^{\mathrm{AB}} \frac{\partial f_{\mathrm{A}}}{\partial x^{1}} \frac{\partial f_{\mathrm{B}}}{\partial x^{\mathrm{k}}}, \mathrm{e} \\
\left\{P_{\mathrm{j}}, P_{\mathrm{k}}\right\}_{D\left(\Phi^{*}\right)} & =\left\{P_{\mathrm{j}}, P_{\mathrm{k}}\right\} \\
& -\left\{P_{\mathrm{j}}, f_{\mathrm{A}}(x)\right\} A^{\mathrm{AS}} \delta^{\mathrm{rs}} P^{\mathrm{l}}\left(\frac{\partial^{2} f_{\mathrm{S}}}{\partial x^{\mathrm{r}} \partial x^{1}} \frac{\partial f_{\mathrm{R}}}{\partial x^{\mathrm{s}}}-\frac{\partial f_{\mathrm{S}}}{\partial x^{\mathrm{s}}} \frac{\partial^{2} f_{\mathrm{R}}}{\partial x^{\mathrm{r}} \partial x^{1}}\right) A^{\mathrm{RB}}\left\{f_{\mathrm{B}}(x), P_{\mathrm{k}}\right\} \\
& +\left\{P_{\mathrm{j}}, f_{\mathrm{A}}(x)\right\} A^{\mathrm{AB}} P^{1}\left\{\frac{\partial f_{\mathrm{B}}}{\partial x^{1}}, P_{\mathrm{k}}\right\}-P^{1}\left\{P_{\mathrm{j}}, \frac{\partial f_{\mathrm{A}}}{\partial x^{1}}\right\} A^{\mathrm{AB}}\left\{f_{\mathrm{B}}(x), P_{\mathrm{k}}\right\} \\
& =P^{1} A^{\mathrm{AS}} A^{\mathrm{RB}} \delta^{\mathrm{rs}}\left(\frac{\partial^{2} f_{\mathrm{S}}}{\partial x^{\mathrm{r}} \partial x^{1}} \frac{\partial f_{\mathrm{R}}}{\partial x^{\mathrm{s}}}-\frac{\partial f_{\mathrm{S}}}{\partial x^{\mathrm{s}}} \frac{\partial^{2} f_{\mathrm{R}}}{\partial x^{\mathrm{r}} \partial x^{1}}\right) \frac{\partial f_{\mathrm{A}}}{\partial x^{\mathrm{j}}} \frac{\partial f_{\mathrm{B}}}{\partial x^{\mathrm{k}}} \\
& -P^{1} A^{\mathrm{AB}}\left(\frac{\partial f_{\mathrm{A}}}{\partial x^{\mathrm{j}}} \frac{\partial^{2} f_{\mathrm{B}}}{\partial x^{\mathrm{k}} \partial x^{1}}-\frac{\partial^{2} f_{\mathrm{A}}}{\partial x^{\mathrm{j}} \partial x^{1}} \frac{\partial f_{\mathrm{B}}}{\partial x^{\mathrm{k}}}\right) \cdot
\end{aligned}
$$

Em especial, o peculiar uso de funções $\mathcal{F}_{\mathrm{jkl}}: \mathbb{R}^{\mathrm{D}+\mathrm{N}} \rightarrow \mathbb{R}$ dadas por

$$
\begin{aligned}
\mathcal{F}_{j \mathrm{kl}}(x) & =-A^{\mathrm{AB}}\left(\frac{\partial f_{\mathrm{A}}}{\partial x^{\mathrm{j}}} \frac{\partial^{2} f_{\mathrm{B}}}{\partial x^{\mathrm{k}} \partial x^{1}}-\frac{\partial^{2} f_{\mathrm{A}}}{\partial x^{\mathrm{j}} \partial x^{1}} \frac{\partial f_{\mathrm{B}}}{\partial x^{\mathrm{k}}}\right) \\
& +A^{\mathrm{AS}} A^{\mathrm{RB}} \delta^{\mathrm{rs}}\left(\frac{\partial^{2} f_{\mathrm{S}}}{\partial x^{\mathrm{r}} \partial x^{1}} \frac{\partial f_{\mathrm{R}}}{\partial x^{\mathrm{s}}}-\frac{\partial f_{\mathrm{S}}}{\partial x^{\mathrm{s}}} \frac{\partial^{2} f_{\mathrm{R}}}{\partial x^{\mathrm{r}} \partial x^{1}}\right) \frac{\partial f_{\mathrm{A}}}{\partial x^{\mathrm{j}}} \frac{\partial f_{\mathrm{B}}}{\partial x^{\mathrm{k}}}
\end{aligned}
$$


permitem ao último comutador ser reescrito $\mathrm{como}^{8}$

$$
\left\{P_{\mathrm{j}}, P_{\mathrm{k}}\right\}_{D\left(\Phi^{*}\right)}=P^{1} \mathcal{F}_{\mathrm{jkl}}(x)
$$

\subsection{Observações importantes}

Embora a Hamiltoniana obtida em (3.7) não contenha quaisquer produtos entre termos dependentes de $x$ e $P$, e portanto pareça ser um bom ponto de partida para o procedimento de quantização haja vista que, após a "promoção" destas mesmas variáveis a operadores quânticos, um operador Hamiltoniano autoadjunto fica definido de modo único, devemos frisar que a teoria quântica assim obtida também não é liberta dos problemas relacionados ao estabelecimento não ambíguo de operadores autoadjuntos. Isso é devido, por exemplo, à aparição de produtos com dependência nas variáveis $x$ e $P$ no conjunto de vínculos responsáveis pela definição do fibrado cotangente $T^{*} \mathcal{M}$ onde o movimento da partícula se descreve, especificamente em $\Phi_{\mathrm{N}+\mathrm{A}}^{*}$. Ou seja, essas ambiguidades não sumiram: elas foram apenas transferidas de um lugar para outro.

Conforme os dizeres do capítulo anterior, a consideração do $\mathbb{R}^{\mathrm{D}+\mathrm{N}}$ parametrizado por $Q=$ $\left(q^{1}, \ldots, q^{\mathrm{D}}, \mathcal{Q}^{1}, \ldots, \mathcal{Q}^{\mathrm{N}}\right)$ em algum dos seus abertos permite descrever a subvariedade $\mathcal{M}$ como

$$
\mathcal{Q}^{\mathrm{A}}=f^{\mathrm{A}}(x)=0
$$

Deste modo, uma vez que $\left\{\partial / \partial q^{1}, \ldots, \partial / \partial q^{\mathrm{D}}\right\}$ e $\left\{\partial / \partial \mathcal{Q}^{1}, \ldots, \partial / \partial \mathcal{Q}^{\mathrm{N}}\right\}$ servem como as respectivas bases para os planos tangente e normal a $\mathcal{M}$ num dos seus pontos, notamos que ambas as parametrizações do $T^{*} \mathbb{R}^{\mathrm{D}+\mathrm{N}}$ se relacionam necessariamente por

$$
x^{\mathrm{j}}=x^{\mathrm{j}}(Q) \quad, P^{\mathrm{j}}=p^{\mu} \frac{\partial x^{\mathrm{j}}}{\partial q^{\mu}}+\mathcal{P}^{\mathrm{A}} \frac{\partial x^{\mathrm{j}}}{\partial \mathcal{Q}^{\mathrm{A}}}
$$

Assim, visto a restrição de $P$ ao plano tangente de $\mathcal{M}$ apresentada em (3.10), obtemos

$$
\Omega^{\mathrm{A}}(Q, P)=\mathcal{Q}^{\mathrm{A}}=0, \Omega^{\mathrm{N}+\mathrm{A}}(Q, P)=\mathcal{P}^{\mathrm{A}}=0
$$

como os vínculos do sistema físico sob esta nova parametrização, os quais são identicamente nulos quaisquer sejam os valores assumidos pelo par $\omega=(q, p)$ de conjugadas. Portanto, ao longo da

\footnotetext{
${ }^{8}$ Este resultado será útil no próximo capítulo. Aliás dele, podemos identificar $\mathcal{F}_{j \mathrm{kl}}(x)$ como as "constantes" que estruturam a álgebra de Lie aqui relacionada.
} 
subvariedade $T^{*} \mathcal{M}$, temos

$$
\begin{aligned}
x^{j} & =x^{j}(q), \\
P^{j} & =\left.p^{\mu} \frac{\partial x^{j}}{\partial q^{\mu}}\right|_{\mathcal{Q}=0} \Rightarrow P_{j}=p_{\nu} g^{\nu \mu}(q)\left(\delta_{j \mathrm{k}} \frac{\partial x^{\mathrm{k}}}{\partial q^{\mu}}\right)_{\mathcal{Q}=0} \Rightarrow P_{j}=\left.p_{\mu} \frac{\partial q^{\mu}}{\partial x^{\mathrm{j}}}\right|_{\mathcal{Q}=0},
\end{aligned}
$$

uma vez que

$$
g^{\mu \nu}(q)=\left.\delta^{\mathrm{jk}} \frac{\partial q^{\mu}}{\partial x^{\mathrm{j}}} \frac{\partial q^{\nu}}{\partial x^{\mathrm{k}}}\right|_{\mathcal{Q}=0}
$$

são as componentes do tensor métrico induzido na subvariedade $\mathcal{M}$, face o processo de imersão isométrica ${ }^{9}$.

Logo, através do particular uso dessas considerações em (3.7) e tomando $\mathcal{V}(q)=V(x(q))$, observa-se

$$
H(\omega)=\frac{1}{2 m} \delta^{\mathrm{jk}}\left[\left(p_{\mu} \frac{\partial q^{\mu}}{\partial x^{\mathrm{j}}}\right)\left(p_{\nu} \frac{\partial q^{\nu}}{\partial x^{\mathrm{k}}}\right)\right]_{\mathcal{Q}=0}+V(x(q))=\frac{1}{2 m} g^{\mu \nu}(q) p_{\mu} p_{\nu}+\mathcal{V}(q)
$$

como a Hamiltoniana $H: T^{*} \mathcal{M} \rightarrow \mathbb{R}$ associada à partícula em estudo. Desta maneira, identificando $\mathcal{V}(q)$ com o potencial da Hamiltoniana (1.2), notamos que a dinâmica do sistema físico obtida com o uso de uma teoria de sistemas vinculados equivale a original. Ou seja, do ponto de vista clássico é indiferente considerar o sistema físico num espaço Riemanniano ou vinculado a uma superfície regular num espaço Euclideano dimensionalmente maior, fato também observado em [21].

\footnotetext{
${ }^{9}$ Vide o capítulo anterior, especialmente as Seções 2.3 e 2.4 .
} 


\section{Capítulo 4}

\section{O processo de quantização}

\subsection{Alguns comentários}

Conforme mencionado anteriormente, a construção de uma teoria quântica para qualquer sistema físico que se restringe a uma variedade $\mathcal{M}$, possuindo representação clássica Hamiltoniana, é feita pelo estabelecimento de uma correspondência entre a álgebra constituída pelas funções definidas no fibrado cotangente $T^{*} \mathcal{M}$, diretamente associadas aos observáveis da teoria clássica, e aquela relacionada aos operadores quânticos necessariamente autoadjuntos, atuantes nos vetores de um espaço de Hilbert $\mathfrak{H}$ complexo $[5,34]$.

Quando lidamos com uma teoria Hamiltoniana particularmente ambientada no $T^{*} \mathbb{R}^{\mathrm{D}+\mathrm{N}}$, onde figura um conjunto formado exclusivamente por 2N vínculos de segunda classe, responsável pelo estabelecimento da subvariedade $T^{*} \mathcal{M}$ na qual o sistema físico reside, esta mesma correspondência se constrói em obediência ao formalismo de Schrödinger através da "promoção" específica dos parâmetros z, responsáveis pela descrição de $T^{*} \mathbb{R}^{\mathrm{D}+\mathrm{N}}$, a operadores $\hat{z}$ autoadjuntos obedientes às relações de comutação [7]

$$
\left[\hat{\mathbf{z}}^{\mathrm{A}}, \hat{\mathbf{z}}^{\mathrm{B}}\right]=\left.\left.i \hbar\left\{\mathbf{z}^{\mathrm{A}}, \mathbf{z}^{\mathrm{B}}\right\}_{D(\Phi)}\right|_{\mathbf{z} \rightarrow \hat{\mathbf{z}}}\right|_{\hat{\Phi}=0}
$$

Por uma simples consequência, todas as funções correspondentes aos observáveis físicos da teoria clássica Hamiltoniana também se "promovem" a operadores quânticos autoadjuntos satisfazendo relações análogas de comutação ${ }^{1}$. Um bom exemplo disso é o operador Hamiltoniano $\hat{H}$ que obrigatoriamente dá-se por $\hat{H}=H(\hat{\mathbf{z}})$ remontando, através da consideração de $\varphi$ como o vetor de estado representativo do sistema físico quanticamente, que a evolução temporal do mesmo satisfaz

\footnotetext{
${ }^{1}$ Especificamente, quaisquer funções clássicas $\mathcal{F}, \mathcal{G}: T^{*} \mathcal{M} \rightarrow \mathbb{R}$ são "promovidas" aos respectivos operadores $\hat{F}$ e $\hat{G}$ satisfazendo a

$$
[\hat{F}, \hat{G}]=[\mathcal{F}(\hat{z}), \mathcal{G}(\hat{\mathbf{z}})]=\left.\left.i \hbar\{\mathcal{F}(\mathbf{z}), \mathcal{G}(\mathrm{z})\}_{D(\Phi)}\right|_{z \rightarrow \hat{z}}\right|_{\hat{\Phi}=0} .
$$


a relação

$$
\hat{H} \varphi=i \hbar \frac{\partial \varphi}{\partial t}
$$

Entretanto devemos reforçar, mais uma vez, que o estabelecimento de uma teoria quântica nestes moldes não ocorre necessariamente de modo único. Isso se deve a eventual não comutatividade dos operadores envolvidos em (4.1) frente à parametrização adotada, haja vista que deles depende o completo estabelecimento da correspondência suprarreferida entre os observáveis da teoria clássica e os operadores necessariamente autoadjuntos da teoria quântica almejada, em obediência a (4.2). Aliás, de acordo com todos os resultados e comentários anteriores, a descrição do sistema em análise neste trabalho, quando feita não apenas por uma $\omega=(q, p)$ simplética remontando à formulação Hamiltoniana (1.2), mas por outra $z=(x, P)$ que estrutura a Hamiltoniana (3.7), figura como um perfeito exemplo desta situação, mediante à presença de produtos clássicos entre parâmetros canonicamente conjugados em algumas funções físicas: basta ver a própria (1.2), e a expressão para os vínculos (3.16) e comutadores (3.20) oriundos da última parametrização.

Apesar disso, e perante à cômoda observação que a Hamiltoniana (3.7) é liberta dos problemas referentes ao estabelecimento de um único operador Hamiltoniano sob os moldes supracitados, realizaremos o processo de quantização sobre a teoria clássica obtida em termos de $z$, apesar desta também apresentar a mesma "patia" relacionada à ambiguidade no ordenamento dos operadores. Neste caso em específico, o operador Hamiltoniano se define univocamente por

$$
\hat{H}=H(\hat{\mathbf{z}})=\frac{1}{2 m} \delta^{\mathrm{jk}} \hat{P}_{\mathrm{j}} \hat{P}_{\mathrm{k}}+V(\hat{x})
$$

\subsection{O ordenamento de operadores}

Lembrando que o produto entre dois operadores autoadjuntos $\hat{A}_{1}$ e $\hat{A}_{2}$ não necessariamente será autoadjunto ${ }^{2}$, podemos construir um terceiro operador $\hat{B}$, certamente autoadjunto, por meio do ordenamento simétrico

$$
\hat{B}=a \hat{A}_{1} \hat{A}_{2}+a^{*} \hat{A}_{2} \hat{A}_{1}
$$

sendo $a$ um número complexo qualquer e $a^{*}$ o seu complexo conjugado. De um modo geral, quando temos um produto de $\mathrm{n}$ operadores autoadjuntos, a definição de outro $\hat{B}^{\prime}$ também autoadjunto pode ser feita mediante a soma de produtos dos $\mathrm{n}$ operadores ordenados simetricamente, onde

\footnotetext{
${ }^{2}$ Particularmente, $\hat{A}_{1} \hat{A}_{2}$ será autoadjunto se $\hat{A}_{1}$ e $\hat{A}_{2}$ comutarem.
} 
(4.4) é um caso particular. Explicitamente temos

$$
\hat{B}^{\prime}=a_{\mathrm{m}} \hat{B}^{\mathrm{m}}+a_{\mathrm{m}}^{*} \hat{B}^{\mathrm{m} \dagger}
$$

com $\hat{B}^{\mathrm{m}}$ sendo o m-ésimo produto possível envolvendo os operadores $\hat{A}_{1}, \ldots, \hat{A}_{\mathrm{n}}$ sem repetição.

No particular caso da quantização de uma teoria clássica estruturada para um sistema físico, além da preocupação referente à autoadjunticidade dos operadores quânticos, existe outra de vital importância: a validade do princípio da correspondência ${ }^{3}$. Deste modo por exemplo, ao considerarmos uma relação de "um-pra-um" entre as funções $\mathcal{A}_{1}, \ldots, \mathcal{A}_{\mathrm{n}}$ da teoria clássica e os respectivos operadores quânticos autoadjuntos $\hat{A}_{1}, \ldots, \hat{A}_{\mathrm{n}}$, uma teoria quântica se completa quando é necessária a associação do produto de algumas das n funções $\mathcal{A}_{1}, \ldots, \mathcal{A}_{\mathrm{n}}$ ao operador $\hat{B}^{\prime}$ autoadjunto por um ordenamento simétrico, tal como o estruturado em (4.5), e desde que, pelo menos, a soma dos coeficientes desta expressão seja igual a unidade; ou seja,

$$
2 \sum_{j=1}^{\mathrm{m}} \operatorname{Re}\left(a_{j}\right)=1 .
$$

Dado a infinitude de soluções para esta igualdade, percebemos a priori a não unicidade na definição de teorias quânticas para sistemas físicos com representação clássica, onde figuram produtos entre variáveis canonicamente conjugadas, uma vez que as últimas são "promovidas" a operadores autoadjuntos pelo processo de quantização.

Apenas para concluir estes comentários, devemos mencionar que, para sistemas clássicos descritos por parâmetros canonicamente conjugados $q$ e $p$, talvez uma das mais conhecidas escolhas no que se refere ao ordenamento dos operadores de uma teoria quântica, face aos requerimentos de autoadjunticidade, é atribuída a Hermann Weyl. De um modo geral, o seu procedimento consiste em associar uma função clássica $\mathcal{A}: T^{*} \mathcal{M} \rightarrow \mathbb{R}$ a um operador autoadjunto $\hat{A}$ dado por [35]

$$
\hat{A}=(2 \pi \hbar)^{-2 \mathrm{D}} \int \mathcal{A}(q, p) e^{\frac{i}{\hbar}[(q-\hat{Q}) u+(p-\hat{P}) v]} d u d v d q d p
$$

\footnotetext{
${ }^{3}$ No caso aqui mencionado, este príncipio se refere ao fato da correspondência Q, mencionada na Seção 1.1, ter limite clássico: ou seja, no limite em que $\hbar \rightarrow 0$ recaímos nas mesmas observações clássicas. Isso pode ser traduzido nos termos gerais de uma teoria singular como

$$
\lim _{\hbar \rightarrow 0}\left\{\frac{1}{i \hbar}\left[\hat{A}_{1}, \hat{A}_{2}\right]\right\}=\left.\left\{\mathcal{A}_{1}, \mathcal{A}_{2}\right\}_{D(\Phi)}\right|_{\Phi=0}
$$

onde $\mathcal{A}_{1}, \mathcal{A}_{2}: T^{*} \mathcal{M} \rightarrow \mathbb{R}$ são duas funções clássicas, tais que $\mathrm{Q}\left(\mathcal{A}_{1}\right)=\hat{A}_{1}$ e $\mathrm{Q}\left(\mathcal{A}_{2}\right)=\hat{A}_{2}$.
} 
onde 2D se associa à dimensão de $T^{*} \mathcal{M}$. Apesar da não aparência, os operadores obtidos pela quantização de Weyl figuram como casos particulares do ordenamento resumido em (4.5), sendo $a_{1}, \ldots, a_{\mathrm{m}}$ números necessariamente reais. Apenas como um exemplo, podemos mencionar o caso de uma função clássica $\mathcal{A}: T^{*} \mathcal{M} \rightarrow \mathbb{R}$ expressa por

$$
\mathcal{A}(q, p)=\mathcal{G}(q) p^{\mathrm{n}}
$$

com $\mathcal{G}: \mathcal{M} \rightarrow \mathbb{R}$, sendo $\mathrm{n}$ um número natural: adotando a prescrição de Weyl, o operador autoadjunto a ela correspondente se define como $^{4}$

$$
\hat{A}=\frac{1}{2^{\mathrm{n}}} \sum_{\mathrm{j}=0}^{\mathrm{n}}\left(\begin{array}{l}
\mathrm{n} \\
\mathrm{j}
\end{array}\right)(\hat{p})^{\mathrm{j}} \mathcal{G}(\hat{q})(\hat{p})^{\mathrm{n}-\mathrm{j}}
$$

de onde é notável o respeito às relações (4.5) e (4.6).

Com o efeito destas observações gerais, uma candidata para a quantização da teoria presente no capítulo anterior, já possuindo o operador Hamiltoniano (4.3), se completa tendo em vista que, além dos operadores $\hat{x}$ e $\hat{P}$ obrigatoriamente satisfazerem $\mathrm{a}^{5}$

$$
\left[\hat{x}^{\mathrm{j}}, \hat{x}^{\mathrm{k}}\right]=0 \quad, \quad\left[\hat{x}^{\mathrm{j}}, \hat{P}_{\mathrm{k}}\right]=i \hbar\left(\delta_{\mathrm{k}}^{\mathrm{j}}-\delta^{\mathrm{j} 1} A^{\mathrm{AB}} \frac{\partial f_{\mathrm{A}}}{\partial x^{\mathrm{l}}} \frac{\partial f_{\mathrm{B}}}{\partial x^{\mathrm{k}}}\right)_{\star}
$$

uma vez que as expressões clássicas aqui associadas se mostram livres de quaisquer produtos entre parâmetros canonicamente conjugados, eles também deve ser tais que

$$
\left[\hat{P}_{\mathrm{j}}, \hat{P}_{\mathrm{k}}\right]=i \hbar\left(a \hat{P}^{1} \hat{F}_{\mathrm{jk} 1}+a^{*} \hat{F}_{\mathrm{jk1}} \hat{P}^{1}\right)_{\hat{\Phi}=0}
$$

sendo a um número complexo e $\hat{F}=\mathcal{F}(\hat{x})$ o correspondente quântico associado a $\mathcal{F}(x)$, perante o fato deste comutador ter de remontar a um operador necessariamente autoadjunto ${ }^{6}$. Convém

\footnotetext{
${ }^{4}$ Vide [35] para a demonstração deste resultado em particular.

${ }^{5}$ Por questões de simplicidade, subentenderemos que as funções indexadas por $\star$ se referem a operadores quânticos obtidos pela promoção dos parâmetros do $\mathbb{R}^{\mathrm{D}+\mathrm{N}}$ a operadores $\hat{x}$, adicionalmente submissos aos vínculos quânticos $\hat{\Phi}=0$ mencionados na sequência.

${ }^{6}$ Devemos frisar que a escolha por um único a fez-se por questões de simplicidade: a escolha mais geral deve associar um complexo $a_{\mathrm{m}}$ a cada possibilidade de ordenamento, conforme observamos anteriormente e em respeito a (4.6), visto o conhecimento explícito da expressão clássica $\mathcal{F}_{j \mathrm{kl}}(x)$ e face à nítida possibilidade de expressá-la como um produtório de outras duas funções também definidas no $\mathbb{R}^{\mathrm{D}+\mathrm{N}}$.

Entretanto, apesar deste ordenamento figurar como um caso particular, devemos frisar que no caso de $a$ ser um número real, trata-se da única escolha existente para este caso em específico, visto que todas as demais recaem a ela. Para observar este fato basta tomarmos, como simples exemplo, $\mathcal{F}(x)=\mathcal{A}_{1}(x) \mathcal{A}_{2}(x)$ : isso remete-nos
} 
salientar que

$$
a=\frac{1}{2}+i r \quad, \text { com } r \in \mathbb{R},
$$

face a necessária obediência a (4.6).

Analogamente, haja vista que os vínculos (3.16) são essenciais à definição da teoria clássica, dado o comprometimento destes para com a plena definição da subvariedade onde o sistema se define, os operadores a eles associados na teoria quântica se expressam por ${ }^{7}$

$$
\hat{\Phi}_{\mathrm{A}}^{*}=f_{\mathrm{A}}(\hat{x})=0, \hat{\Phi}_{\mathrm{N}+\mathrm{A}}^{*}=\left.b \hat{P}^{\mathrm{j}} \frac{\partial f_{\mathrm{A}}}{\partial x^{\mathrm{j}}}\right|_{\hat{x}}+\left.b^{*} \frac{\partial f_{\mathrm{A}}}{\partial x^{\mathrm{j}}}\right|_{\hat{x}} \hat{P}^{\mathrm{j}}=0
$$

com $b$ se definindo pelos mesmos motivos suprarreferidos através de

$$
b=\frac{1}{2}+i r \quad \text { onde } r \in \mathbb{R} .
$$

\subsection{Relações canônicas de comutação}

Apesar de já termos "em mãos" esta teoria, seria interessante convertê-la à outra, onde os operadores satisfazendo a (4.1) tornar-se-iam canonicamente $\operatorname{conjugados}^{8}$, intentando avaliar, por exemplo, quais as eventuais consequências que o ato de mergulhar um espaço curvo isometricamente em outro Euclideano traz à formulação quântica: trata-se de algo factível, perante à existência de um relacionamento bem estabelecido entre as parametrizações $x$ e $Q$ intrínsecas ao $\mathbb{R}^{\mathrm{D}+\mathrm{N}}$ numa das vizinhanças nele presentes, conforme o mencionado nos capítulos anteriores. Por se dizer, ambas as parametrizações do fibrado cotangente $T^{*} \mathbb{R}^{\mathrm{D}+\mathrm{N}}$ são relacionadas por

$$
Q^{\mathrm{j}}=\bar{Q}^{\mathrm{j}}(x), p_{\mathrm{j}}=\bar{p}_{\mathrm{j}}(Q, P)=P_{\mathrm{k}} \frac{\partial x^{\mathrm{k}}}{\partial Q^{\mathrm{j}}}
$$

especificamente a

$$
\begin{aligned}
& a \mathcal{A}_{1}(\hat{x}) \hat{P} \mathcal{A}_{2}(\hat{x})+a \mathcal{A}_{2}(\hat{x}) \hat{P} \mathcal{A}_{1}(\hat{x})= \\
& \quad=a \hat{P} \mathcal{F}(\hat{x})+a\left[\mathcal{A}_{1}(\hat{x}), \hat{P}\right] \mathcal{A}_{2}(\hat{x})+a \mathcal{F}(\hat{x}) \hat{P}+a \mathcal{A}_{2}(\hat{x})\left[\hat{P}, \mathcal{A}_{1}(\hat{x})\right]=a \hat{P} \mathcal{F}(\hat{x})+a \mathcal{F}(\hat{x}) \hat{P}
\end{aligned}
$$

frente à independência de (4.7) dos produtos entre operadores conjugados.

${ }^{7}$ Apenas para reforçar: aqui estamos assumindo, com o respaudo nas observações feitas para quantizações, que para cada função clássica, liberta dos produtos entre parâmetros canonicamente conjugados, já está associado um único operador autoadjunto. Deste modo, resta-nos definir os demais operadores autoadjuntos, correspondentes às funções clássicas não libertas dos mesmos produtos, através do ordenamento dos operadores quânticos já bem estabelecidos.

${ }^{8} \mathrm{Ou}$ seja, obedientes à condição de irredutibilidade mencionada em (1.1). 
de onde, através da escolha de $Q=(q, \mathcal{Q})$, sendo $q$ as coordenadas intrínsecas a $\mathcal{M}$, se torna possível verificar que, especificamente em $\mathcal{M}$, vale

$$
q^{\mu}=\bar{q}^{\mu}(x) \quad, \quad \mathcal{Q}^{\mathrm{A}}=\overline{\mathcal{Q}}^{\mathrm{A}}(x)=0 \quad, p_{\mu}=\bar{p}_{\mu}(x, P)=\left.P_{\mathrm{j}} \frac{\partial x^{\mathrm{j}}}{\partial q^{\mu}}\right|_{\mathcal{Q}=0} \quad, \quad \mathcal{P}_{\mathrm{A}}=\overline{\mathcal{P}}_{\mathrm{A}}(x, P)=0
$$

No caso, as equações $\mathcal{Q}(x)=0$ são as responsáveis por caracterizar $\mathcal{M}$ como uma subvariedade, as quais adicionalmente se identificam com (3.1).

Inspirados nestas observações, e em adicional respeito às recomendações feitas para o estabelecimento de operadores numa teoria quântica diante apenas da necessidade de ordenamentos, definiremos um par de operadores $\hat{\omega}=(\hat{q}, \hat{p})$ autoadjuntos em função daqueles resumidos no par $\hat{z}$ supracitado através de

$$
\hat{q}^{\mu}=q^{\mu}(\hat{x}) \quad, \hat{p}_{\mu}=p_{\mu}(\hat{x}, \hat{P})=\left.\left(\frac{1}{2}+i d\right) \hat{P}_{j} \frac{\partial x^{j}}{\partial q^{\mu}}\right|_{\star}+\left.\left(\frac{1}{2}-i d\right) \frac{\partial x^{j}}{\partial q^{\mu}}\right|_{\star} \hat{P}_{j},
$$

sendo $d$ um número necessariamente real, cabendo-nos avaliar se estes operadores já figuram como canonicamente conjugados e se, por exemplo, já satisfazem plenamente o princípio da correspondência, ou se existem condições específicas para isso.

Através do especial acordo entre o primeiro destes operadores com a expressão (4.1) e a propriedade (D.5) dos parênteses de Dirac, é possível observar que

$$
\begin{aligned}
{\left[\hat{q}^{\mu}, \hat{q}^{\nu}\right] } & =\left[q^{\mu}(\hat{x}), q^{\nu}(\hat{x})\right]=\left.i \hbar\left\{q^{\mu}(x), q^{\nu}(x)\right\}_{D\left(\Phi^{*}\right)}\right|_{\star} \\
& =\left.i \hbar \frac{\partial q^{\mu}}{\partial x^{\mathrm{j}}} \frac{\partial q^{\nu}}{\partial x^{\mathrm{k}}}\left\{x^{\mathrm{j}}, x^{\mathrm{k}}\right\}_{D\left(\Phi^{*}\right)}\right|_{\star}=\left.\frac{\partial q^{\mu}}{\partial x^{\mathrm{j}}} \frac{\partial q^{\nu}}{\partial x^{\mathrm{k}}}\right|_{\star}\left[\hat{x}^{\mathrm{j}}, \hat{x}^{\mathrm{k}}\right]=0 .
\end{aligned}
$$

Aliás, pelo mesmo raciocínio também temos

$$
\begin{aligned}
{\left[\hat{q}^{\mu}, \hat{p}_{\nu}\right] } & =\left(\frac{1}{2}+i d\right)\left[q^{\mu}(\hat{x}),\left.\frac{\partial x^{\mathrm{k}}}{\partial q^{\nu}}\right|_{\star} \hat{P}_{\mathrm{k}}\right]+\left(\frac{1}{2}-i d\right)\left[q^{\mu}(\hat{x}),\left.\hat{P}_{\mathrm{k}} \frac{\partial x^{\mathrm{k}}}{\partial q^{\nu}}\right|_{\star}\right] \\
& =\left.\left(\frac{1}{2}+i d\right) \frac{\partial x^{\mathrm{k}}}{\partial q^{\nu}}\right|_{\star}\left[q^{\mu}(\hat{x}), \hat{P}_{\mathrm{k}}\right]+\left.\left(\frac{1}{2}-i d\right)\left[q^{\mu}(\hat{x}), \hat{P}_{\mathrm{k}}\right] \frac{\partial x^{\mathrm{k}}}{\partial q^{\nu}}\right|_{\star},
\end{aligned}
$$

de onde o fato de que

$$
\left[q^{\mu}(\hat{x}), \hat{P}_{\mathrm{k}}\right]=\left.i \hbar\left\{q^{\mu}(x), P_{\mathrm{k}}\right\}_{D\left(\Phi^{*}\right)}\right|_{\hat{z}}=\left.i \hbar \frac{\partial q^{\mu}}{\partial x^{\mathrm{j}}}\left\{x^{\mathrm{j}}, P_{\mathrm{k}}\right\}_{D\left(\Phi^{*}\right)}\right|_{\star}
$$


permite-nos reduzir a mesma (4.12) em

$$
\begin{aligned}
{\left[\hat{q}^{\mu}, \hat{p}_{\nu}\right] } & =i \hbar \frac{\partial q^{\mu}}{\partial x^{\mathrm{j}}} \frac{\partial x^{\mathrm{k}}}{\partial q^{\nu}}\left(\delta_{\mathrm{k}}^{\mathrm{j}}-\delta^{\mathrm{j} 1} A^{\mathrm{AB}} \frac{\partial \mathcal{Q}_{\mathrm{A}}}{\partial x^{1}} \frac{\partial \mathcal{Q}_{\mathrm{B}}}{\partial x^{\mathrm{k}}}\right)_{\star} \\
& =\left.i \hbar \delta_{\mathrm{k}}^{\mathrm{j}} \frac{\partial q^{\mu}}{\partial x^{\mathrm{j}}} \frac{\partial x^{\mathrm{k}}}{\partial q^{\nu}}\right|_{\star}-i \hbar A^{\mathrm{AB}} \frac{\partial \mathcal{Q}_{\mathrm{B}}}{\partial x^{\mathrm{k}}} \frac{\partial x^{\mathrm{k}}}{\partial q^{\nu}}\left(\delta^{\mathrm{j} 1} \frac{\partial q^{\mu}}{\partial x^{\mathrm{j}}} \frac{\partial \mathcal{Q}_{\mathrm{A}}}{\partial x^{1}}\right)_{\star}=i \hbar \hat{\delta}_{\nu}^{\mu}
\end{aligned}
$$

visto a validade de (2.10). Desta maneira perante os dois últimos resultados, resta-nos obter o resultado de $\left[\hat{p}_{\mu}, \hat{p}_{\nu}\right]$ para avaliarmos a canonicidade do par de operadores $\hat{\omega}$.

\subsubsection{Um cálculo honestamente enfadonho}

Uma vez que essa restrição à expressão (4.10) para o operador momento permite notar que

$$
\begin{aligned}
{\left[\hat{p}_{\mu}, \hat{p}_{\nu}\right] } & =\left(\frac{1}{4}-d^{2}+i d\right)\left[\left.\hat{P}_{\mathrm{j}} \frac{\partial x^{\mathrm{j}}}{\partial q^{\mu}}\right|_{\star},\left.\hat{P}_{\mathrm{k}} \frac{\partial x^{\mathrm{k}}}{\partial q^{\nu}}\right|_{\star}\right]_{+}+\left(\frac{1}{4}+d^{2}\right)\left[\left.\hat{P}_{\mathrm{j}} \frac{\partial x^{\mathrm{j}}}{\partial q^{\mu}}\right|_{\star},\left.\frac{\partial x^{\mathrm{k}}}{\partial q^{\nu}}\right|_{\star} \hat{P}_{\mathrm{k}}\right] \\
& +\left(\frac{1}{4}+d^{2}\right)\left[\left.\frac{\partial x^{\mathrm{j}}}{\partial q^{\mu}}\right|_{\star} \hat{P}_{\mathrm{j}},\left.\hat{P}_{\mathrm{k}} \frac{\partial x^{\mathrm{k}}}{\partial q^{\nu}}\right|_{\star}\right]_{+}+\left(\frac{1}{4}-d^{2}-i d\right)\left[\left.\frac{\partial x^{\mathrm{j}}}{\partial q^{\mu}}\right|_{\star} \hat{P}_{\mathrm{j}},\left.\frac{\partial x^{\mathrm{k}}}{\partial q^{\nu}}\right|_{\star} \hat{P}_{\mathrm{k}}\right],
\end{aligned}
$$

o peculiar uso das relações

$$
\begin{aligned}
& \left.\left.\frac{\partial x^{\mathrm{j}}}{\partial q^{\mu}}\right|_{\star} \hat{P}_{\mathrm{j}} \frac{\partial x^{\mathrm{k}}}{\partial q^{\nu}}\right|_{\star} \hat{P}_{\mathrm{k}}=\left.\frac{\partial x^{\mathrm{j}}}{\partial q^{\mu}}\right|_{\star}\left[\hat{P}_{\mathrm{j}},\left.\frac{\partial x^{\mathrm{k}}}{\partial q^{\nu}}\right|_{\star}\right]_{\hat{P}_{\mathrm{k}}}+\left.\frac{\partial x^{\mathrm{j}}}{\partial q^{\mu}} \frac{\partial x^{\mathrm{k}}}{\partial q^{\nu}}\right|_{\star} \hat{P}_{\mathrm{j}} \hat{P}_{\mathrm{k}}, \\
& \left.\hat{P}_{j} \frac{\partial x^{\mathrm{j}}}{\partial q^{\mu}} \frac{\partial x^{\mathrm{k}}}{\partial q^{\nu}}\right|_{\star} \hat{P}_{\mathrm{k}}=\left.\left[\hat{P}_{\mathrm{j}},\left.\frac{\partial x^{\mathrm{j}}}{\partial q^{\mu}}\right|_{\star}\right] \frac{\partial x^{\mathrm{k}}}{\partial q^{\nu}}\right|_{\star} \hat{P}_{\mathrm{k}}+\left.\left.\frac{\partial x^{\mathrm{j}}}{\partial q^{\mu}}\right|_{\star} \hat{P}_{\mathrm{j}} \frac{\partial x^{\mathrm{k}}}{\partial q^{\nu}}\right|_{\star} \hat{P}_{\mathrm{k}}
\end{aligned}
$$

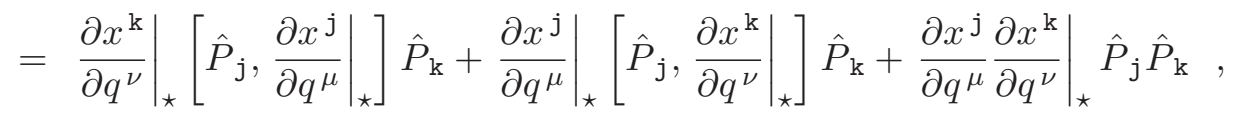

$$
\begin{aligned}
& \left.\left.\frac{\partial x^{\mathrm{j}}}{\partial q^{\mu}}\right|_{\star} \hat{P}_{\mathrm{j}} \hat{P}_{\mathrm{k}} \frac{\partial x^{\mathrm{k}}}{\partial q^{\nu}}\right|_{\star}=\left.\frac{\partial x^{\mathrm{j}}}{\partial q^{\mu}}\right|_{\star} \hat{P}_{\mathrm{j}}\left[\hat{P}_{\mathrm{k}},\left.\frac{\partial x^{\mathrm{k}}}{\partial q^{\nu}}\right|_{\star}\right]_{+}+\left.\left.\frac{\partial x^{\mathrm{j}}}{\partial q^{\mu}}\right|_{\star} \hat{P}_{\mathrm{j}} \frac{\partial x^{\mathrm{k}}}{\partial q^{\nu}}\right|_{\star} \hat{P}_{\mathrm{k}} \\
& =\left.\frac{\partial x^{\mathrm{j}}}{\partial q^{\mu}}\right|_{\star}\left[\hat{P}_{\mathrm{j}},\left[\hat{P}_{\mathrm{k}},\left.\frac{\partial x^{\mathrm{k}}}{\partial q^{\nu}}\right|_{\star}\right]\right]+\left.\frac{\partial x^{\mathrm{j}}}{\partial q^{\mu}}\right|_{\star}\left[\hat{P}_{\mathrm{k}},\left.\frac{\partial x^{\mathrm{k}}}{\partial q^{\nu}}\right|_{\star}\right]_{\hat{P}_{\mathrm{j}}}
\end{aligned}
$$

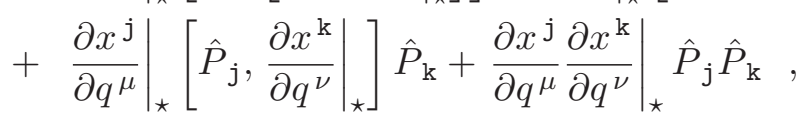




$$
\begin{aligned}
& \left.\left.\hat{P}_{\mathrm{j}} \frac{\partial x^{\mathrm{j}}}{\partial q^{\mu}}\right|_{\star} \hat{P}_{\mathrm{k}} \frac{\partial x^{\mathrm{k}}}{\partial q^{\nu}}\right|_{\star}=\left\{\left.\left[\hat{P}_{\mathrm{j}},\left.\frac{\partial x^{\mathrm{j}}}{\partial q^{\mu}}\right|_{\star}\right]_{+} \frac{\partial x^{\mathrm{j}}}{\partial q^{\mu}}\right|_{\star} \hat{P}_{\mathrm{j}}\right\}\left\{\left[\hat{P}_{\mathrm{k}},\left.\frac{\partial x^{\mathrm{k}}}{\partial q^{\nu}}\right|_{\star}\right]+\left.\frac{\partial x^{\mathrm{k}}}{\partial q^{\nu}}\right|_{\star} \hat{P}_{\mathrm{k}}\right\} \\
& =\left[\hat{P}_{\mathrm{j}},\left.\frac{\partial x^{\mathrm{j}}}{\partial q^{\mu}}\right|_{\star}\right]\left[\hat{P}_{\mathrm{k}},\left.\frac{\partial x^{\mathrm{k}}}{\partial q^{\nu}}\right|_{\star}\right]_{+}+\left.\left[\hat{P}_{\mathrm{j}},\left.\frac{\partial x^{\mathrm{j}}}{\partial q^{\mu}}\right|_{\star}\right] \frac{\partial x^{\mathrm{k}}}{\partial q^{\nu}}\right|_{\star} \hat{P}_{\mathrm{k}} \\
& +\left.\frac{\partial x^{\mathrm{j}}}{\partial q^{\mu}}\right|_{\star} \hat{P}_{\mathrm{j}}\left[\hat{P}_{\mathrm{k}},\left.\frac{\partial x^{\mathrm{k}}}{\partial q^{\nu}}\right|_{\star}\right]+\left.\left.\frac{\partial x^{\mathrm{j}}}{\partial q^{\mu}}\right|_{\star} \hat{P}_{\mathrm{j}} \frac{\partial x^{\mathrm{k}}}{\partial q^{\nu}}\right|_{\star} \hat{P}_{\mathrm{k}}
\end{aligned}
$$

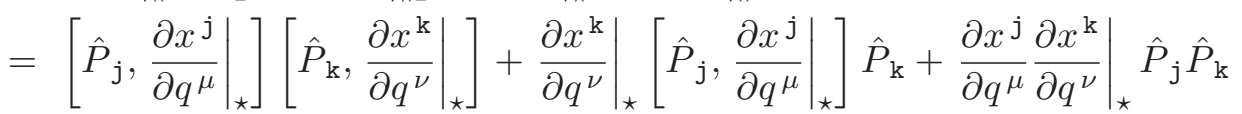

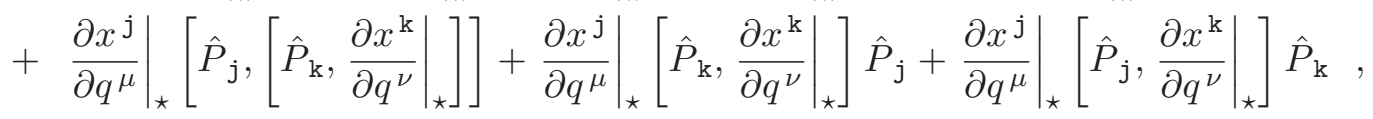

mostra-nos a imediata redução do mesmo comutador em

$$
\begin{aligned}
{\left[\hat{p}_{\mu}, \hat{p}_{\nu}\right] } & =\left(\frac{1}{2}+i d\right)\left\{\left.\frac{\partial x^{\mathrm{j}}}{\partial q^{\mu}}\right|_{\star}\left[\hat{P}_{\mathrm{j}},\left[\hat{P}_{\mathrm{k}},\left.\frac{\partial x^{\mathrm{k}}}{\partial q^{\nu}}\right|_{\star}\right]\right]-\left.\frac{\partial x^{\mathrm{k}}}{\partial q^{\nu}}\right|_{\star}\left[\hat{P}_{\mathrm{k}},\left[\hat{P}_{\mathrm{j}},\left.\frac{\partial x^{\mathrm{j}}}{\partial q^{\mu}}\right|_{\star}\right]\right]\right\} \\
& +\left.\frac{\partial x^{\mathrm{j}}}{\partial q^{\mu}}\right|_{\star}\left[\hat{P}_{\mathrm{j}},\left.\frac{\partial x^{\mathrm{k}}}{\partial q^{\nu}}\right|_{\star}\right]_{\hat{P}_{\mathrm{k}}}-\left.\frac{\partial x^{\mathrm{k}}}{\partial q^{\nu}}\right|_{\star}\left[\hat{P}_{\mathrm{k}},\left.\frac{\partial x^{\mathrm{j}}}{\partial q^{\mu}}\right|_{\star}\right]_{\hat{P}_{j}}+\left.\frac{\partial x^{\mathrm{j}}}{\partial q^{\mu}} \frac{\partial x^{\mathrm{k}}}{\partial q^{\nu}}\right|_{\star}\left[\hat{P}_{j}, \hat{P}_{\mathrm{k}}\right]
\end{aligned}
$$

resultado sobre o qual trabalharemos por partes.

\section{A extinção do primeiro termo}

Com efeito da segunda relação em (4.7) e das propriedades dos parênteses de Dirac presentes no Apêndice A, notamos especialmente que

$$
\begin{aligned}
{\left[\hat{P}_{\mathrm{j}},\left.\frac{\partial x^{\mathrm{k}}}{\partial q^{\nu}}\right|_{\star}\right] } & =i \hbar\left\{P_{\mathrm{j}}, \frac{\partial x^{\mathrm{k}}}{\partial q^{\nu}}\right\}_{D\left(\Phi^{*}\right)}=-\left.i \hbar \frac{\partial q^{\gamma}}{\partial x^{1}} \frac{\partial^{2} x^{\mathrm{k}}}{\partial q^{\gamma} \partial q^{\nu}}\left\{x^{1}, P_{\mathrm{j}}\right\}_{D\left(\Phi^{*}\right)}\right|_{\star} \\
& =-i \hbar \frac{\partial q^{\gamma}}{\partial x^{1}} \frac{\partial^{2} x^{\mathrm{k}}}{\partial q^{\gamma} \partial q^{\nu}}\left(\delta_{\mathrm{j}}^{1}-\delta^{\mathrm{la}} A^{\mathrm{AB}} \frac{\partial \mathcal{Q}_{\mathrm{A}}}{\partial x^{\mathrm{a}}} \frac{\partial \mathcal{Q}_{\mathrm{B}}}{\partial x^{\mathrm{j}}}\right)_{\star}=-\left.i \hbar \frac{\partial q^{\gamma}}{\partial x^{\mathrm{j}}} \frac{\partial^{2} x^{\mathrm{k}}}{\partial q^{\gamma} \partial q^{\nu}}\right|_{\star} \\
& +i \hbar A^{\mathrm{AB}} \frac{\partial \mathcal{Q}_{\mathrm{B}}}{\partial x^{\mathrm{j}}} \frac{\partial^{2} x^{\mathrm{k}}}{\partial q^{\gamma} \partial q^{\nu}}\left(\delta^{\mathrm{la}} \frac{\partial q^{\gamma}}{\partial x^{\mathrm{l}}} \frac{\partial \mathcal{Q}_{\mathrm{A}}}{\partial x^{\mathrm{a}}}\right)_{\star}=-\left.i \hbar \frac{\partial q^{\gamma}}{\partial x^{\mathrm{j}}} \frac{\partial^{2} x^{\mathrm{k}}}{\partial q^{\gamma} \partial q^{\nu}}\right|_{\star},
\end{aligned}
$$

de onde obtemos, por exemplo,

$$
\left.\frac{\partial x^{\mathrm{j}}}{\partial q^{\mu}}\right|_{\star}\left[\hat{P}_{\mathrm{j}},\left.\frac{\partial x^{\mathrm{k}}}{\partial q^{\nu}}\right|_{\star}\right]_{\hat{P}_{\mathrm{k}}}=-\left.i \hbar \delta_{\mu}^{\gamma} \frac{\partial^{2} x^{\mathrm{k}}}{\partial q^{\gamma} \partial q^{\nu}}\right|_{\star} \hat{P}_{\mathrm{k}}=-\left.i \hbar \frac{\partial^{2} x^{\mathrm{k}}}{\partial q^{\mu} \partial q^{\nu}}\right|_{\star} \hat{P}_{\mathrm{k}}
$$

Deste modo, em decorrência da simetria envolta neste último resultado, se torna claro que

$$
\left.\frac{\partial x^{\mathrm{j}}}{\partial q^{\mu}}\right|_{\star}\left[\hat{P}_{\mathrm{j}},\left.\frac{\partial x^{\mathrm{k}}}{\partial q^{\nu}}\right|_{\star}\right]_{\hat{P}_{\mathrm{k}}}-\left.\frac{\partial x^{\mathrm{k}}}{\partial q^{\nu}}\right|_{\star}\left[\hat{P}_{\mathrm{k}},\left.\frac{\partial x^{\mathrm{j}}}{\partial q^{\mu}}\right|_{\star}\right]_{\hat{P}_{\mathrm{j}}=0}
$$




\section{A extinção do segundo termo}

Já da observação que a mesma (4.15) remonta a

$$
\begin{aligned}
& {\left[\hat{P}_{\mathrm{j}},\left[\hat{P}_{\mathrm{k}}, \frac{\partial x^{\mathrm{k}}}{\partial q^{\nu}} \mid\right]\right]=-i \hbar\left[\hat{P}_{\mathrm{j}},\left.\frac{\partial q^{\gamma}}{\partial x^{\mathrm{k}}} \frac{\partial^{2} x^{\mathrm{k}}}{\partial q^{\gamma} \partial q^{\nu}}\right|_{\star}\right]} \\
& =\left.\hbar^{2}\left\{P_{\mathrm{j}}, \frac{\partial q^{\gamma}}{\partial x^{\mathrm{k}}} \frac{\partial^{2} x^{\mathrm{k}}}{\partial q^{\gamma} \partial q^{\nu}}\right\}_{D\left(\Phi^{\star}\right)}\right|_{\hat{z}}=-\left.\hbar^{2} \frac{\partial}{\partial x^{1}}\left(\frac{\partial q^{\gamma}}{\partial x^{\mathrm{k}}} \frac{\partial^{2} x^{\mathrm{k}}}{\partial q^{\gamma} \partial q^{\nu}}\right)\left\{x^{1}, P_{\mathrm{j}}\right\}_{D\left(\Phi^{\star}\right)}\right|_{\hat{z}} \\
& =-\hbar^{2}\left(\delta_{\mathrm{j}}^{1}-\delta^{\mathrm{la}} A^{\mathrm{AB}} \frac{\partial \mathcal{Q}_{\mathrm{A}}}{\partial x^{\mathrm{a}}} \frac{\partial \mathcal{Q}_{\mathrm{B}}}{\partial x^{\mathrm{j}}}\right) \frac{\partial}{\partial x^{1}}\left(\frac{\partial q^{\gamma}}{\partial x^{\mathrm{k}}} \frac{\partial^{2} x^{\mathrm{k}}}{\partial q^{\gamma} \partial q^{\nu}}\right)_{\star} \\
& =-\hbar^{2} \frac{\partial}{\partial x^{\mathrm{j}}}\left(\frac{\partial q^{\gamma}}{\partial x^{\mathrm{k}}} \frac{\partial^{2} x^{\mathrm{k}}}{\partial q^{\gamma} \partial q^{\nu}}\right)_{\star}+\hbar^{2} A^{\mathrm{AB}} \frac{\partial \mathcal{Q}_{\mathrm{B}}}{\partial x^{\mathrm{j}}}\left[\delta^{\mathrm{al}} \frac{\partial \mathcal{Q}_{\mathrm{A}}}{\partial x^{\mathrm{a}}} \frac{\partial}{\partial x^{1}}\left(\frac{\partial q^{\gamma}}{\partial x^{\mathrm{k}}} \frac{\partial^{2} x^{\mathrm{k}}}{\partial q^{\gamma} \partial q^{\nu}}\right)\right]_{\star} \\
& =-\hbar^{2} \frac{\partial}{\partial x^{\mathrm{j}}}\left(\frac{\partial q^{\gamma}}{\partial x^{\mathrm{k}}} \frac{\partial^{2} x^{\mathrm{k}}}{\partial q^{\gamma} \partial q^{\nu}}\right)_{\star}+\left.\hbar^{2} A^{\mathrm{AB}} \frac{\partial \mathcal{Q}_{\mathrm{B}}}{\partial x^{\mathrm{j}}}\left(\delta^{\mathrm{al}} \frac{\partial \mathcal{Q}_{\mathrm{A}}}{\partial x^{\mathrm{a}}} \frac{\partial^{2} q^{\gamma}}{\partial x^{1} \partial x^{\mathrm{k}}}\right) \frac{\partial^{2} x^{\mathrm{k}}}{\partial q^{\gamma} \partial q^{\nu}}\right|_{\star},
\end{aligned}
$$

também obtemos, por exemplo, que

$$
\begin{aligned}
& \left.\frac{\partial x^{\mathrm{j}}}{\partial q^{\mu}}\right|_{\star}\left[\hat{P}_{\mathrm{j}},\left[\hat{P}_{\mathrm{k}},\left.\frac{\partial x^{\mathrm{k}}}{\partial q^{\nu}}\right|_{\star}\right]\right]=-\hbar^{2} \frac{\partial}{\partial q^{\mu}}\left(\frac{\partial q^{\gamma}}{\partial x^{\mathrm{k}}} \frac{\partial^{2} x^{\mathrm{k}}}{\partial q^{\gamma} \partial q^{\nu}}\right)_{\star} \\
& \quad+\left.\hbar^{2} A^{\mathrm{AB}} \frac{\partial x^{\mathrm{j}}}{\partial q^{\mu}} \frac{\partial \mathcal{Q}_{\mathrm{B}}}{\partial x^{\mathrm{j}}}\left(\delta^{\mathrm{al}} \frac{\partial \mathcal{Q}_{\mathrm{A}}}{\partial x^{\mathrm{a}}} \frac{\partial^{2} q^{\gamma}}{\partial x^{1} \partial x^{\mathrm{k}}}\right) \frac{\partial^{2} x^{\mathrm{k}}}{\partial q^{\gamma} \partial q^{\nu}}\right|_{\star}=-\hbar^{2} \frac{\partial}{\partial q^{\mu}}\left(\frac{\partial q^{\gamma}}{\partial x^{\mathrm{k}}} \frac{\partial^{2} x^{\mathrm{k}}}{\partial q^{\gamma} \partial q^{\nu}}\right)_{\star} .
\end{aligned}
$$

Desta maneira, frente à constatação que o tensor métrico $g: \mathcal{X}(\mathcal{M}) \times \mathcal{X}(\mathcal{M}) \rightarrow \mathcal{D}(\mathcal{M})$ definido em (2.6) permite-nos identificar o termo sob derivação como ${ }^{9}$

$$
\frac{\partial q^{\gamma}}{\partial x^{\mathrm{j}}} \frac{\partial^{2} x^{\mathrm{j}}}{\partial q^{\gamma} \partial q^{\mu}}=\frac{1}{2} \frac{\partial}{\partial q^{\mu}} \ln \mathrm{g}
$$

é notável das duas últimas igualdades que

$$
\left.\frac{\partial x^{\mathrm{j}}}{\partial q^{\mu}}\right|_{\star}\left[\hat{P}_{\mathrm{j}},\left[\hat{P}_{\mathrm{k}},\left.\frac{\partial x^{\mathrm{k}}}{\partial q^{\nu}}\right|_{\star}\right]\right]-\left.\frac{\partial x^{\mathrm{k}}}{\partial q^{\nu}}\right|_{\star}\left[\hat{P}_{\mathrm{k}},\left[\hat{P}_{\mathrm{j}},\left.\frac{\partial x^{\mathrm{j}}}{\partial q^{\mu}}\right|_{\star}\right]\right]=0
$$

face às mesmas considerações de simetria mencionadas anteriormente para a obtenção de (4.16). Logo de ambos resultados, temos a necessária redução do comutador (4.14) em

$$
\left[\hat{p}_{\mu}, \hat{p}_{\nu}\right]=\left.\frac{\partial x^{\mathrm{j}}}{\partial q^{\mu}} \frac{\partial x^{\mathrm{k}}}{\partial q^{\nu}}\right|_{\star}\left[\hat{P}_{\mathrm{j}}, \hat{P}_{\mathrm{k}}\right]
$$

\footnotetext{
${ }^{9}$ Vide o Apêndice C, não apenas para maiores detalhes sobre este resultado, através da expressão (C.5), mas para informações acerca do que se resume em $\mathcal{X}(\mathcal{M})$ e $\mathcal{D}(\mathcal{M})$.
} 
de onde o particular emprego de (4.8) mostra-nos

$$
\begin{aligned}
{\left[\hat{p}_{\mu}, \hat{p}_{\nu}\right] } & =-\left.\hbar \frac{\partial x^{\mathrm{j}}}{\partial q^{\mu}} \frac{\partial x^{\mathrm{k}}}{\partial q^{\nu}}\right|_{\star}\left[\left(s-\frac{i}{2}\right) \hat{P}^{1} \hat{F}_{\mathrm{jkl}}+\left(s+\frac{i}{2}\right) \hat{F}_{\mathrm{jkl}} \hat{P}^{\mathrm{I}}\right] \\
& =-\left.\hbar \frac{\partial x^{\mathrm{j}}}{\partial q^{\mu}} \frac{\partial x^{\mathrm{k}}}{\partial q^{\nu}}\right|_{\star}\left\{\left(s-\frac{i}{2}\right)\left[\hat{P}^{1}, \hat{F}_{\mathrm{jkl}}\right]+2 s \hat{F}_{\mathrm{jkl}} \hat{P}^{1}\right\}
\end{aligned}
$$

sendo $s$ um número real remontando a $a=1 / 2+i s$, conforme o mencionado em (4.8).

\section{A canonicidade dos operadores}

Perante à observação de que

$$
\begin{gathered}
\left.\frac{\partial x^{\mathrm{j}}}{\partial q^{\mu}} \frac{\partial x^{\mathrm{k}}}{\partial q^{\nu}}\right|_{\star} \hat{F}_{j \mathrm{kl}}=A^{\mathrm{AB}} \frac{\partial x^{\mathrm{j}}}{\partial q^{\mu}} \frac{\partial^{2} \mathcal{Q}_{\mathrm{A}}}{\partial x^{\mathrm{j}} \partial x^{1}}\left(\frac{\partial x^{\mathrm{k}}}{\partial q^{\nu}} \frac{\partial \mathcal{Q}_{\mathrm{B}}}{\partial x^{\mathrm{k}}}\right)_{\star}-A^{\mathrm{AB}} \frac{\partial x^{\mathrm{k}}}{\partial q^{\nu}} \frac{\partial^{2} \mathcal{Q}_{\mathrm{B}}}{\partial x^{\mathrm{k}} \partial x^{1}}\left(\frac{\partial x^{\mathrm{j}}}{\partial q^{\mu}} \frac{\partial \mathcal{Q}_{\mathrm{A}}}{\partial x^{\mathrm{j}}}\right)_{\star} \\
+A^{\mathrm{AS}} A^{\mathrm{RB}} \delta^{\mathrm{rs}}\left(\frac{\partial^{2} \mathcal{Q}_{\mathrm{S}}}{\partial x^{\mathrm{r}} \partial x^{1}} \frac{\partial \mathcal{Q}_{\mathrm{R}}}{\partial x^{\mathrm{s}}}-\frac{\partial \mathcal{Q}_{\mathrm{S}}}{\partial x^{\mathrm{s}}} \frac{\partial^{2} \mathcal{Q}_{\mathrm{R}}}{\partial x^{\mathrm{r}} \partial x^{1}}\right)\left(\frac{\partial x^{\mathrm{j}}}{\partial q^{\mu}} \frac{\partial \mathcal{Q}_{\mathrm{A}}}{\partial x^{\mathrm{j}}}\right)\left(\frac{\partial x^{\mathrm{k}}}{\partial q^{\nu}} \frac{\partial \mathcal{Q}_{\mathrm{B}}}{\partial x^{\mathrm{k}}}\right)_{\star}=0
\end{gathered}
$$

segue a imediata redução do último comutador como

$$
\begin{aligned}
{\left[\hat{p}_{\mu}, \hat{p}_{\nu}\right] } & =\left.\hbar \frac{\partial x^{\mathrm{j}}}{\partial q^{\mu}} \frac{\partial x^{\mathrm{k}}}{\partial q^{\nu}}\right|_{\star}\left(s-\frac{i}{2}\right) \delta^{\mathrm{la}}\left[\hat{F}_{\mathrm{jk} 1}, \hat{P}_{\mathrm{a}}\right]=\left.\hbar^{2} \frac{\partial x^{\mathrm{j}}}{\partial q^{\mu}} \frac{\partial x^{\mathrm{k}}}{\partial q^{\nu}}\left(\frac{1}{2}+i s\right) \delta^{\mathrm{la}}\left\{\mathcal{F}_{\mathrm{jkl}}, P_{\mathrm{a}}\right\}_{D\left(\Phi^{*}\right)}\right|_{\star} \\
& =\left.a \hbar^{2} \frac{\partial x^{\mathrm{j}}}{\partial q^{\mu}} \frac{\partial x^{\mathrm{k}}}{\partial q^{\nu}} \delta^{\mathrm{la}} \frac{\partial \mathcal{F}_{\mathrm{jkl}}}{\partial x^{b}}\left\{x^{b}, P_{\mathrm{a}}\right\}_{D\left(\Phi^{*}\right)}\right|_{\star} \\
& =a \hbar^{2} \frac{\partial x^{\mathrm{j}}}{\partial q^{\mu}} \frac{\partial x^{\mathrm{k}}}{\partial q^{\nu}} \delta^{1 \mathrm{a}} \frac{\partial \mathcal{F}_{\mathrm{jk}}}{\partial x^{b}}\left(\delta_{\mathrm{a}}^{\mathrm{b}}-\delta^{\mathrm{bn}} A^{\mathrm{RS}} \frac{\partial \mathcal{Q}_{\mathrm{R}}}{\partial x^{\mathrm{n}}} \frac{\partial \mathcal{Q}_{\mathrm{S}}}{\partial x^{\mathrm{a}}}\right)_{\star} .
\end{aligned}
$$

Assim, com o efeito de

$$
\begin{aligned}
& \left.\frac{\partial \mathcal{F}_{j \mathrm{kl}}}{\partial x^{b}}\right|_{\star}=-\frac{\partial A^{\mathrm{AB}}}{\partial x^{\mathrm{b}}}\left(\frac{\partial \mathcal{Q}_{\mathrm{A}}}{\partial x^{\mathrm{j}}} \frac{\partial^{2} \mathcal{Q}_{\mathrm{B}}}{\partial x^{\mathrm{k}} \partial x^{1}}-\frac{\partial^{2} \mathcal{Q}_{\mathrm{A}}}{\partial x^{\mathrm{j}} \partial x^{1}} \frac{\partial \mathcal{Q}_{\mathrm{B}}}{\partial x^{\mathrm{k}}}\right)_{\star} \\
& -A^{\mathrm{AB}}\left(\frac{\partial^{2} \mathcal{Q}_{\mathrm{A}}}{\partial x^{\mathrm{b}} \partial x^{\mathrm{j}}} \frac{\partial^{2} \mathcal{Q}_{\mathrm{B}}}{\partial x^{\mathrm{k}} \partial x^{1}}+\frac{\partial \mathcal{Q}_{\mathrm{A}}}{\partial x^{\mathrm{j}}} \frac{\partial^{3} \mathcal{Q}_{\mathrm{B}}}{\partial x^{\mathrm{b}} \partial x^{\mathrm{k}} \partial x^{1}}-\frac{\partial^{3} \mathcal{Q}_{\mathrm{A}}}{\partial x^{\mathrm{b}} \partial x^{\mathrm{j}} \partial x^{1}} \frac{\partial \mathcal{Q}_{\mathrm{B}}}{\partial x^{\mathrm{k}}}-\frac{\partial^{2} \mathcal{Q}_{\mathrm{A}}}{\partial x^{\mathrm{j}} \partial x^{1}} \frac{\partial^{2} \mathcal{Q}_{\mathrm{B}}}{\partial x^{\mathrm{b}} \partial x^{\mathrm{k}}}\right)_{\star} \\
& +\left(\frac{\partial}{\partial x^{\mathrm{b}}} A^{\mathrm{AS}} A^{\mathrm{RB}}\right) \delta^{\mathrm{rs}} \frac{\partial \mathcal{Q}_{\mathrm{A}}}{\partial x^{\mathrm{j}}} \frac{\partial \mathcal{Q}_{\mathrm{B}}}{\partial x^{\mathrm{k}}}\left(\frac{\partial^{2} \mathcal{Q}_{\mathrm{S}}}{\partial x^{\mathrm{r}} \partial x^{1}} \frac{\partial \mathcal{Q}_{\mathrm{R}}}{\partial x^{\mathrm{s}}}-\frac{\partial \mathcal{Q}_{\mathrm{S}}}{\partial x^{\mathrm{s}}} \frac{\partial^{2} \mathcal{Q}_{\mathrm{R}}}{\partial x^{\mathrm{r}} \partial x^{1}}\right)_{\star}+\left.A^{\mathrm{AS}} A^{\mathrm{RB}} \frac{\partial \mathcal{Q}_{\mathrm{A}}}{\partial x^{\mathrm{j}}} \frac{\partial \mathcal{Q}_{\mathrm{B}}}{\partial x^{\mathrm{k}}}\right|_{\star} \\
& \times \delta^{\mathrm{rs}}\left(\frac{\partial^{3} \mathcal{Q}_{\mathrm{S}}}{\partial x^{\mathrm{b}} \partial x^{\mathrm{r}} \partial x^{1}} \frac{\partial \mathcal{Q}_{\mathrm{R}}}{\partial x^{\mathrm{s}}}+\frac{\partial^{2} \mathcal{Q}_{\mathrm{S}}}{\partial x^{\mathrm{r}} \partial x^{1}} \frac{\partial^{2} \mathcal{Q}_{\mathrm{R}}}{\partial x^{\mathrm{b}} \partial x^{\mathrm{s}}}-\frac{\partial^{2} \mathcal{Q}_{\mathrm{S}}}{\partial x^{\mathrm{b}} \partial x^{\mathrm{s}}} \frac{\partial^{2} \mathcal{Q}_{\mathrm{R}}}{\partial x^{\mathrm{r}} \partial x^{1}}-\frac{\partial \mathcal{Q}_{\mathrm{S}}}{\partial x^{\mathrm{s}}} \frac{\partial^{3} \mathcal{Q}_{\mathrm{R}}}{\partial x^{\mathrm{b}} \partial x^{\mathrm{r}} \partial x^{1}}\right)_{\star} \\
& +A^{\mathrm{AS}} A^{\mathrm{RB}} \delta^{\mathrm{rs}}\left(\frac{\partial^{2} \mathcal{Q}_{\mathrm{S}}}{\partial x^{\mathrm{r}} \partial x^{\mathrm{l}}} \frac{\partial \mathcal{Q}_{\mathrm{R}}}{\partial x^{\mathrm{s}}}-\frac{\partial \mathcal{Q}_{\mathrm{S}}}{\partial x^{\mathrm{s}}} \frac{\partial^{2} \mathcal{Q}_{\mathrm{R}}}{\partial x^{\mathrm{r}} \partial x^{\mathrm{l}}}\right)\left(\frac{\partial^{2} \mathcal{Q}_{\mathrm{A}}}{\partial x^{\mathrm{b}} \partial x^{\mathrm{j}}} \frac{\partial \mathcal{Q}_{\mathrm{B}}}{\partial x^{\mathrm{k}}}+\frac{\partial \mathcal{Q}_{\mathrm{A}}}{\partial x^{\mathrm{j}}} \frac{\partial^{2} \mathcal{Q}_{\mathrm{B}}}{\partial x^{\mathrm{b}} \partial x^{\mathrm{k}}}\right)_{\star}
\end{aligned}
$$


revelar que

$$
\begin{aligned}
& \left.\frac{\partial x^{\mathrm{j}}}{\partial q^{\mu}} \frac{\partial x^{\mathrm{k}}}{\partial q^{\nu}} \frac{\partial \mathcal{F}_{j \mathrm{kl}}}{\partial x^{b}}\right|_{\star}= \\
& =\frac{\partial A^{\mathrm{AB}}}{\partial x^{\mathrm{b}}}\left[\frac{\partial x^{\mathrm{j}}}{\partial q^{\mu}} \frac{\partial^{2} \mathcal{Q}_{\mathrm{A}}}{\partial x^{\mathrm{j}} \partial x^{1}}\left(\frac{\partial x^{\mathrm{k}}}{\partial q^{\nu}} \frac{\partial \mathcal{Q}_{\mathrm{B}}}{\partial x^{\mathrm{k}}}\right)-\left(\frac{\partial x^{\mathrm{j}}}{\partial q^{\mu}} \frac{\partial \mathcal{Q}_{\mathrm{A}}}{\partial x^{\mathrm{j}}}\right) \frac{\partial x^{\mathrm{k}}}{\partial q^{\nu}} \frac{\partial^{2} \mathcal{Q}_{\mathrm{B}}}{\partial x^{\mathrm{k}} \partial x^{1}}\right]_{\star} \\
& +A^{\mathrm{AB}} \frac{\partial x^{\mathrm{j}}}{\partial q^{\mu}} \frac{\partial x^{\mathrm{k}}}{\partial q^{\nu}}\left(\frac{\partial^{2} \mathcal{Q}_{\mathrm{A}}}{\partial x^{\mathrm{j}} \partial x^{\mathrm{l}}} \frac{\partial^{2} \mathcal{Q}_{\mathrm{B}}}{\partial x^{\mathrm{b}} \partial x^{\mathrm{k}}}-\frac{\partial^{2} \mathcal{Q}_{\mathrm{A}}}{\partial x^{\mathrm{b}} \partial x^{\mathrm{j}}} \frac{\partial^{2} \mathcal{Q}_{\mathrm{B}}}{\partial x^{\mathrm{k}} \partial x^{\mathrm{l}}}\right)_{\star} \\
& -A^{\mathrm{AB}} \frac{\partial x^{\mathrm{k}}}{\partial q^{\nu}} \frac{\partial^{3} \mathcal{Q}_{\mathrm{B}}}{\partial x^{\mathrm{b}} \partial x^{\mathrm{k}} \partial x^{1}}\left(\frac{\partial x^{\mathrm{j}}}{\partial q^{\mu}} \frac{\partial \mathcal{Q}_{\mathrm{A}}}{\partial x^{\mathrm{j}}}\right)_{\star}+A^{\mathrm{AB}} \frac{\partial x^{\mathrm{j}}}{\partial q^{\mu}} \frac{\partial^{3} \mathcal{Q}_{\mathrm{A}}}{\partial x^{\mathrm{b}} \partial x^{\mathrm{j}} \partial x^{1}}\left(\frac{\partial x^{\mathrm{k}}}{\partial q^{\nu}} \frac{\partial \mathcal{Q}_{\mathrm{B}}}{\partial x^{\mathrm{k}}}\right)_{\star} \\
& +\delta^{\mathrm{rs}}\left(\frac{\partial}{\partial x^{\mathrm{b}}} A^{\mathrm{AS}} A^{\mathrm{RB}}\right)\left(\frac{\partial^{2} \mathcal{Q}_{\mathrm{S}}}{\partial x^{\mathrm{r}} \partial x^{\mathrm{l}}} \frac{\partial \mathcal{Q}_{\mathrm{R}}}{\partial x^{\mathrm{s}}}-\frac{\partial \mathcal{Q}_{\mathrm{S}}}{\partial x^{\mathrm{s}}} \frac{\partial^{2} \mathcal{Q}_{\mathrm{R}}}{\partial x^{\mathrm{r}} \partial x^{\mathrm{l}}}\right)\left(\frac{\partial x^{\mathrm{j}}}{\partial q^{\mu}} \frac{\partial \mathcal{Q}_{\mathrm{A}}}{\partial x^{\mathrm{j}}}\right)\left(\frac{\partial x^{\mathrm{k}}}{\partial q^{\nu}} \frac{\partial \mathcal{Q}_{\mathrm{B}}}{\partial x^{\mathrm{k}}}\right)_{\star} \\
& +A^{\mathrm{AS}} A^{\mathrm{RB}} \delta^{\mathrm{rs}}\left(\frac{\partial^{3} \mathcal{Q}_{\mathrm{S}}}{\partial x^{\mathrm{b}} \partial x^{\mathrm{r}} \partial x^{1}} \frac{\partial \mathcal{Q}_{\mathrm{R}}}{\partial x^{\mathrm{s}}}+\frac{\partial^{2} \mathcal{Q}_{\mathrm{S}}}{\partial x^{\mathrm{r}} \partial x^{1}} \frac{\partial^{2} \mathcal{Q}_{\mathrm{R}}}{\partial x^{\mathrm{b}} \partial x^{\mathrm{s}}}\right)\left(\frac{\partial x^{\mathrm{j}}}{\partial q^{\mu}} \frac{\partial \mathcal{Q}_{\mathrm{A}}}{\partial x^{\mathrm{j}}}\right)\left(\frac{\partial x^{\mathrm{k}}}{\partial q^{\nu}} \frac{\partial \mathcal{Q}_{\mathrm{B}}}{\partial x^{\mathrm{k}}}\right)_{\star} \\
& -A^{\mathrm{AS}} A^{\mathrm{RB}} \delta^{\mathrm{rs}}\left(\frac{\partial^{2} \mathcal{Q}_{\mathrm{S}}}{\partial x^{\mathrm{b}} \partial x^{\mathrm{s}}} \frac{\partial^{2} \mathcal{Q}_{\mathrm{R}}}{\partial x^{\mathrm{r}} \partial x^{1}}+\frac{\partial \mathcal{Q}_{\mathrm{S}}}{\partial x^{\mathrm{s}}} \frac{\partial^{3} \mathcal{Q}_{\mathrm{R}}}{\partial x^{\mathrm{b}} \partial x^{\mathrm{r}} \partial x^{1}}\right)\left(\frac{\partial x^{\mathrm{j}}}{\partial q^{\mu}} \frac{\partial \mathcal{Q}_{\mathrm{A}}}{\partial x^{\mathrm{j}}}\right)\left(\frac{\partial x^{\mathrm{k}}}{\partial q^{\nu}} \frac{\partial \mathcal{Q}_{\mathrm{B}}}{\partial x^{\mathrm{k}}}\right)_{\star} \\
& +A^{\mathrm{AS}} A^{\mathrm{RB}} \delta^{\mathrm{rs}}\left(\frac{\partial^{2} \mathcal{Q}_{\mathrm{S}}}{\partial x^{\mathrm{r}} \partial x^{1}} \frac{\partial \mathcal{Q}_{\mathrm{R}}}{\partial x^{\mathrm{s}}}-\frac{\partial \mathcal{Q}_{\mathrm{S}}}{\partial x^{\mathrm{s}}} \frac{\partial^{2} \mathcal{Q}_{\mathrm{R}}}{\partial x^{\mathrm{r}} \partial x^{1}}\right) \frac{\partial x^{\mathrm{j}}}{\partial q^{\mu}} \frac{\partial^{2} \mathcal{Q}_{\mathrm{A}}}{\partial x^{\mathrm{b}} \partial x^{\mathrm{j}}}\left(\frac{\partial x^{\mathrm{k}}}{\partial q^{\nu}} \frac{\partial \mathcal{Q}_{\mathrm{B}}}{\partial x^{\mathrm{k}}}\right)_{\star} \\
& -\left.A^{\mathrm{AS}} A^{\mathrm{RB}} \delta^{\mathrm{rs}}\left(\frac{\partial^{2} \mathcal{Q}_{\mathrm{S}}}{\partial x^{\mathrm{r}} \partial x^{\mathrm{l}}} \frac{\partial \mathcal{Q}_{\mathrm{R}}}{\partial x^{\mathrm{s}}}-\frac{\partial \mathcal{Q}_{\mathrm{S}}}{\partial x^{\mathrm{s}}} \frac{\partial^{2} \mathcal{Q}_{\mathrm{R}}}{\partial x^{\mathrm{r}} \partial x^{1}}\right)\left(\frac{\partial x^{\mathrm{j}}}{\partial q^{\mu}} \frac{\partial \mathcal{Q}_{\mathrm{A}}}{\partial x^{\mathrm{j}}}\right) \frac{\partial x^{\mathrm{k}}}{\partial q^{\nu}} \frac{\partial^{2} \mathcal{Q}_{\mathrm{B}}}{\partial x^{\mathrm{b}} \partial x^{\mathrm{k}}}\right|_{\star} \\
& =A^{\mathrm{AB}} \frac{\partial x^{\mathrm{j}}}{\partial q^{\mu}} \frac{\partial x^{\mathrm{k}}}{\partial q^{\nu}}\left(\frac{\partial^{2} \mathcal{Q}_{\mathrm{A}}}{\partial x^{\mathrm{j}} \partial x^{1}} \frac{\partial^{2} \mathcal{Q}_{\mathrm{B}}}{\partial x^{\mathrm{b}} \partial x^{\mathrm{k}}}-\frac{\partial^{2} \mathcal{Q}_{\mathrm{A}}}{\partial x^{\mathrm{b}} \partial x^{\mathrm{j}}} \frac{\partial^{2} \mathcal{Q}_{\mathrm{B}}}{\partial x^{\mathrm{k}} \partial x^{1}}\right)_{\star},
\end{aligned}
$$

se torna claro, pela mera substituição deste resultado em (4.18), que

$$
\begin{aligned}
& {\left[\hat{p}_{\mu}, \hat{p}_{\nu}\right] }=a \hbar^{2} \frac{\partial x^{\mathrm{j}}}{\partial q^{\mu}} \frac{\partial x^{\mathrm{k}}}{\partial q^{\nu}} A^{\mathrm{AB}}\left(\frac{\partial^{2} \mathcal{Q}_{\mathrm{A}}}{\partial x^{\mathrm{j}} \partial x^{1}} \frac{\partial^{2} \mathcal{Q}_{\mathrm{B}}}{\partial x^{\mathrm{l}} \partial x^{\mathrm{k}}}-\frac{\partial^{2} \mathcal{Q}_{\mathrm{A}}}{\partial x^{\mathrm{j}} \partial x^{\mathrm{l}}} \frac{\partial^{2} \mathcal{Q}_{\mathrm{B}}}{\partial x^{\mathrm{l}} \partial x^{\mathrm{k}}}\right)_{\star} \\
&-a \hbar^{2} \frac{\partial x^{\mathrm{j}}}{\partial q^{\mu}} \frac{\partial x^{\mathrm{k}}}{\partial q^{\nu}} A^{\mathrm{AB}} A^{\mathrm{RS}}\left(\delta^{1 \mathrm{a}} \frac{\partial^{2} \mathcal{Q}_{\mathrm{A}}}{\partial x^{\mathrm{j}} \partial x^{\mathrm{l}}} \frac{\partial \mathcal{Q}_{\mathrm{S}}}{\partial x^{\mathrm{a}}}\right)\left(\delta^{\mathrm{bn}} \frac{\partial^{2} \mathcal{Q}_{\mathrm{B}}}{\partial x^{\mathrm{k}} \partial x^{\mathrm{b}}} \frac{\partial \mathcal{Q}_{\mathrm{R}}}{\partial x^{\mathrm{n}}}\right)_{\star} \\
&+a \hbar^{2} \frac{\partial x^{\mathrm{j}}}{\partial q^{\mu}} \frac{\partial x^{\mathrm{k}}}{\partial q^{\nu}} A^{\mathrm{AB}} A^{\mathrm{RS}}\left(\delta^{\mathrm{la}} \frac{\partial^{2} \mathcal{Q}_{\mathrm{A}}}{\partial x^{\mathrm{j}} \partial x^{1}} \frac{\partial \mathcal{Q}_{\mathrm{R}}}{\partial x^{\mathrm{a}}}\right)\left(\delta^{\mathrm{bn}} \frac{\partial^{2} \mathcal{Q}_{\mathrm{B}}}{\partial x^{\mathrm{k}} \partial x^{\mathrm{b}}} \frac{\partial \mathcal{Q}_{\mathrm{S}}}{\partial x^{\mathrm{n}}}\right)_{\star}=0
\end{aligned}
$$

face à simetricidade existente na matriz $A$. Deste modo, fazendo-nos valer da representação de posição para teoria quântica aqui em estudo, os operadores canonicamente conjugados se identificam, por exemplo, como

$$
\hat{q}^{\mu}=q^{\mu}, \hat{p}_{\mu}=-i \hbar \mathrm{g}^{-\frac{1}{4}} \partial_{\mu} \mathrm{g}^{\frac{1}{4}}
$$

em acordo aos comentários realizados ao final do Apêndice C.1. 


\subsection{Sobre o operador Hamiltoniano}

Verificada a canonicidade dos operadores presentes em (4.10), se torna possível reexpressar o Hamiltoniano (4.3) em função exclusiva de operadores canonicamente conjugados, perante o isomorfismo suprarreferido entre as parametrizações $x$ e $Q$ do fibrado cotangente $T^{*} \mathbb{R}^{\mathrm{D}+\mathrm{N}}$. Aliás, de acordo com a prescrição anterior para o estabelecimento de operadores autoadjuntos, somos tentados a expressar $\hat{x}$ e $\hat{P}$, ao longo da subvariedade $\mathcal{M}$, em função dos canonicamente conjugados através de

$$
\hat{x}^{j}=x^{j}(\hat{q}) \quad, \quad \hat{P}_{j}=P_{j}(\hat{q}, \hat{p})=\left.\left(\frac{1}{2}+i d^{\prime}\right) \hat{p}_{\mu} \frac{\partial q^{\mu}}{\partial x^{j}}\right|_{\star}+\left.\left(\frac{1}{2}-i d^{\prime}\right) \frac{\partial q^{\mu}}{\partial x^{j}}\right|_{\star} \hat{p}_{\mu}
$$

sendo $d^{\prime}$ um número real, de modo análogo ao feito em (4.10). Porém, trata-se de uma escolha que a priori é inconsistente, uma vez que a própria (4.10) já remonta a uma relação específica entre os operadores $\hat{p}$ e $\hat{P}$, a qual não necessariamente equivale à arbitária (4.19).

Para contornar este problema e estabelecer uma expressão para $\hat{P}$ satisfazendo a (4.10), devemos notar que dela temos

$$
\begin{aligned}
& \left.\hat{p}_{\mu} \frac{\partial q^{\mu}}{\partial x^{\mathrm{j}}}\right|_{\star}=\left(\frac{1}{2}+i d\right) \hat{P}_{\mathrm{j}}+\left.\left(\frac{1}{2}-i d\right) \frac{\partial x^{\mathrm{j}}}{\partial q^{\mu}}\right|_{\star} \hat{P}_{\mathrm{j}} \frac{\partial q^{\mu}}{\partial x^{\mathrm{j}}}=\hat{P}_{\mathrm{j}}+\left.\left(\frac{1}{2}-i d\right)\left[\left.\frac{\partial x^{\mathrm{j}}}{\partial q^{\mu}}\right|_{\star}, \hat{P}_{\mathrm{j}}\right] \frac{\partial q^{\mu}}{\partial x^{\mathrm{j}}}\right|_{\star}, \\
& \left.\frac{\partial q^{\mu}}{\partial x^{\mathrm{j}}}\right|_{\star} \hat{p}_{\mu}=\left.\left(\frac{1}{2}+i d\right) \frac{\partial q^{\mu}}{\partial x^{\mathrm{j}}} \hat{P}_{\mathrm{j}} \frac{\partial x^{\mathrm{j}}}{\partial q^{\mu}}\right|_{\star}+\left(\frac{1}{2}-i d\right) \hat{P}_{\mathrm{j}}=\hat{P}_{\mathrm{j}}-\left.\left(\frac{1}{2}-i d\right)\left[\left.\frac{\partial x^{\mathrm{j}}}{\partial q^{\mu}}\right|_{\star}, \hat{P}_{\mathrm{j}}\right] \frac{\partial q^{\mu}}{\partial x^{\mathrm{j}}}\right|_{\star},
\end{aligned}
$$

de onde segue imediatamente que

$$
\hat{P}_{\mathrm{j}}=\left.\frac{1}{2} \hat{p}_{\mu} \frac{\partial q^{\mu}}{\partial x^{\mathrm{j}}}\right|_{\star}+\left.\frac{1}{2} \frac{\partial q^{\mu}}{\partial x^{\mathrm{j}}}\right|_{\star} \hat{p}_{\mu}-\left.i d\left[\left.\frac{\partial x^{\mathrm{j}}}{\partial q^{\mu}}\right|_{\star}, \hat{P}_{\mathrm{j}}\right] \frac{\partial q^{\mu}}{\partial x^{\mathrm{j}}}\right|_{\star}
$$

Deste modo, para assegurarmos não somente à autoadjunticidade do operador $\hat{P}$, mas também a validade do princípio da correspondência, fica como indicativo tomarmos $d=0$ nesta última igualdade, afinal não parece ser tão claro se, ao tomarmos o limite clássico, $\hat{P}$ obedecerá de fato ao príncipio suprarreferido, uma vez que o comutador originário da não nulidade de $d$ possui um correspondente clássico não nulo ${ }^{10}$. Logo os operadores autoadjuntos $\hat{x}$ e $\hat{P}$ ficam definidos, em

\footnotetext{
${ }^{10}$ Adicionalmente também não podemos negar que soa estranho admitir, por exemplo, um valor de $d$ não nulo haja vista que, se assim o fosse, o operador $\hat{P}$ dependeria da sua própria expressão, a qual intentamos definir.
} 
termos dos canonicamente conjugados $\hat{q}$ e $\hat{p}$, por

$$
\hat{x}^{\mathrm{j}}=x^{\mathrm{j}}(\hat{q}) \quad, \quad \hat{P}_{\mathrm{j}}=P_{\mathrm{j}}(\hat{q}, \hat{p})=\left.\frac{1}{2} \hat{p}_{\mu} \frac{\partial q^{\mu}}{\partial x^{\mathrm{j}}}\right|_{\star}+\left.\frac{1}{2} \frac{\partial q^{\mu}}{\partial x^{\mathrm{j}}}\right|_{\star} \hat{p}_{\mu},
$$

figurando como um caso particular de (4.19), em adicional coincidência com a prescrição de Weyl para o ordenamento de operadores, dependentes de outros canonicamente conjugados, conforme mencionado anteriormente. Aliás devemos observar que nesta situação, frente à invertibilidade da matriz $\tilde{g}$ mencionada em (2.5), as mesmas condições precisam ser impostas em (4.9); ou seja ${ }^{11}$

$$
\hat{\Phi}_{\mathrm{N}+\mathrm{A}}^{*}=\left.\frac{1}{2} \hat{P}^{\mathrm{j}} \frac{\partial f_{\mathrm{A}}}{\partial x^{\mathrm{j}}}\right|_{\hat{x}}+\left.\frac{1}{2} \frac{\partial f_{\mathrm{A}}}{\partial x^{\mathrm{j}}}\right|_{\hat{x}} \hat{P}^{\mathrm{j}}=0
$$

dado que as expressões clássicas a eles correspondentes são as mesmas que originam (4.20).

Adicionalmente, dado a necessária validade do mesmo princípio da correspondência, e baseados na mesma argumentação que nos levou ao resultado (4.20), também podemos notar que o coeficiente $a$, mencionado ao término da seção anterior, deve ser real pois, de acordo com (4.8), se tomássemos $a=1 / 2+i s$, teríamos $^{12}$

$$
\left[\hat{P}_{\mathrm{j}}, \hat{P}_{\mathrm{k}}\right]=i \hbar\left(\frac{1}{2} \hat{P}^{1} \hat{F}_{\mathrm{jk} 1}+\frac{1}{2} \hat{F}_{\mathrm{jk} 1} \hat{P}^{1}\right)_{\hat{\Phi}=0}-s \hbar\left[\hat{P}^{1}, \hat{F}_{\mathrm{jk} 1}\right]
$$

${ }^{11}$ Mais precisamente, esta condição se relaciona ao fato de que

$$
\left.\frac{\partial Q^{\mathrm{j}}}{\partial x^{\mathrm{k}}}\right|_{\mathcal{Q}=0}=\tilde{g}^{\mathrm{ja}}(Q)\left(\delta_{\mathrm{kb}} \frac{\partial x^{\mathrm{b}}}{\partial Q^{\mathrm{a}}}\right)_{\mathcal{Q}=0},
$$

dado a identificação $\hat{\mathcal{P}}_{\mathrm{N}+\mathrm{A}}=\hat{\Phi}_{\mathrm{N}+\mathrm{A}}^{*}$.

${ }^{12}$ No entanto, independente de qualquer contraditoriedade e segundo o observado em [19], todos os comutadores presentes nas últimas igualdades, e que nelas aparecem multiplicados pelos fatores $d, r$ e $s$ (supostos constantes frente às variáveis consideradas), se tornam obrigatoriamente nulos ao tomarmos o limite clássico: no caso estes comutadores se referem apenas às correções quânticas advindas da não comutatividade dos operadores envolvidos. Logo, assumindo que a última perspectiva é verdadeira, a presença de $d, r$ e $s$ não nulos nos ordenamentos aqui efetuados é viável, desde que tratem-se de fatores "bem comportados" ao tomarmos os limites clássicos.

Apesar de todos estes comentários, não entraremos nos pormenores deste assunto, não adotando um ordenamento geral no que segue, principalmente face a um resultado em particular que propomos demonstrar na sequência: ou seja, a teoria quântica que avaliaremos de agora em diante, é dada pelo hamiltoniano (4.3), pelas relações

$$
\left[\hat{x}^{\mathrm{j}}, \hat{x}^{\mathrm{k}}\right]=0 \quad, \quad\left[\hat{x}^{\mathrm{j}}, \hat{P}_{\mathrm{k}}\right]=i \hbar\left(\delta_{\mathrm{k}}^{\mathrm{j}}-\delta^{\mathrm{j} 1} A^{\mathrm{AB}} \frac{\partial f_{\mathrm{A}}}{\partial x^{1}} \frac{\partial f_{\mathrm{B}}}{\partial x^{\mathrm{k}}}\right)_{\star} \quad,\left[\hat{P}_{\mathrm{j}}, \hat{P}_{\mathrm{k}}\right]=\frac{i \hbar}{2}\left(\hat{P}^{1} \hat{F}_{\mathrm{jk} 1}+\hat{F}_{\mathrm{jk} 1} \hat{P}^{1}\right)_{\hat{\Phi}=0}, \mathrm{e}
$$

o condicionamento aos vínculos

$$
\hat{\Phi}_{\mathrm{A}}^{*}=f_{\mathrm{A}}(\hat{x})=0, \hat{\Phi}_{\mathrm{N}+\mathrm{A}}^{*}=\left.\frac{1}{2} \hat{P}^{\mathrm{j}} \frac{\partial f_{\mathrm{A}}}{\partial x^{j}}\right|_{\hat{x}}+\left.\frac{1}{2} \frac{\partial f_{\mathrm{A}}}{\partial x^{j}}\right|_{\hat{x}} \hat{P}^{j}=0 .
$$




\subsubsection{Outro cálculo quase enfadonho}

Através da definição de $\hat{P}$ feita acima, se torna possível observar que $\delta^{\mathrm{jk}} \hat{P}_{\mathrm{j}} \hat{P}_{\mathrm{k}}=\left.\left.\frac{1}{4} \delta^{\mathrm{jk}} \hat{p}_{\mu} \frac{\partial q^{\mu}}{\partial x^{\mathrm{j}}}\right|_{\star} \hat{p}_{\nu} \frac{\partial q^{\nu}}{\partial x^{\mathrm{k}}}\right|_{\star}+\frac{1}{4} \hat{p}_{\mu} \hat{g}^{\mu \nu} \hat{p}_{\nu}+\left.\left.\frac{1}{4} \delta^{\mathrm{jk}} \frac{\partial q^{\mu}}{\partial x^{\mathrm{j}}}\right|_{\star} \hat{p}_{\mu} \hat{p}_{\nu} \frac{\partial q^{\nu}}{\partial x^{\mathrm{k}}}\right|_{\star}+\left.\left.\frac{1}{4} \delta^{\mathrm{jk}} \frac{\partial q^{\mu}}{\partial x^{\mathrm{j}}}\right|_{\star} \hat{p}_{\mu} \frac{\partial q^{\nu}}{\partial x^{\mathrm{j}}}\right|_{\star} \hat{p}_{\nu}$

face à igualdade (2.6). Aliás, uma vez que alguns dos termos presentes se expressam por

$$
\begin{aligned}
& \left.\left.\delta^{\mathrm{jk}} \hat{p}_{\mu} \frac{\partial q^{\mu}}{\partial x^{\mathrm{j}}}\right|_{\star} \hat{p}_{\nu} \frac{\partial q^{\nu}}{\partial x^{\mathrm{k}}}\right|_{\star}= \\
& =\left.\delta^{\mathrm{jk}} \hat{p}_{\mu} \frac{\partial q^{\mu}}{\partial x^{\mathrm{j}}}\right|_{\star}\left[\hat{p}_{\nu},\left.\frac{\partial q^{\nu}}{\partial x^{\mathrm{k}}}\right|_{\star}\right]_{+} \hat{p}_{\mu} \hat{g}^{\mu \nu} \hat{p}_{\nu}=-i \hbar \delta^{\mathrm{jk}} \hat{p}_{\mu} \frac{\partial q^{\mu}}{\partial x^{\mathrm{j}}} \frac{\partial}{\partial q^{\nu}}\left(\frac{\partial q^{\nu}}{\partial x^{\mathrm{k}}}\right)_{\star}+\hat{p}_{\mu} \hat{g}^{\mu \nu} \hat{p}_{\nu}, \\
& \left.\left.\delta^{\mathrm{j}} \frac{\partial q^{\mu}}{\partial x^{\mathrm{j}}}\right|_{\star} \hat{p}_{\mu} \frac{\partial q^{\nu}}{\partial x^{\mathrm{k}}}\right|_{\star} \hat{p}_{\nu}= \\
& =\left.\delta^{\mathrm{jk}}\left\{\left[\left.\frac{\partial q^{\mu}}{\partial x^{\mathrm{j}}}\right|_{\star}, \hat{p}_{\mu}\right]+\left.\hat{p}_{\mu} \frac{\partial q^{\mu}}{\partial x^{\mathrm{j}}}\right|_{\star}\right\} \frac{\partial q^{\nu}}{\partial x^{\mathrm{k}}}\right|_{\star} \hat{p}_{\nu}=i \hbar \delta^{\mathrm{jk}} \frac{\partial q^{\nu}}{\partial x^{\mathrm{k}}} \frac{\partial}{\partial q^{\mu}}\left(\frac{\partial q^{\mu}}{\partial x^{\mathrm{j}}}\right)_{\star} \hat{p}_{\nu}+\hat{p}_{\mu} \hat{g}^{\mu \nu} \hat{p}_{\nu}, \\
& \left.\left.\delta^{\mathrm{jk}} \frac{\partial q^{\mu}}{\partial x^{\mathrm{j}}}\right|_{\star} \hat{p}_{\mu} \hat{p}_{\nu} \frac{\partial q^{\nu}}{\partial x^{\mathrm{k}}}\right|_{\star}= \\
& =\delta^{\mathrm{jk}}\left\{\left.\left[\left.\frac{\partial q^{\mu}}{\partial x^{\mathrm{j}}}\right|_{\star}, \hat{p}_{\mu}\right]_{+} \hat{p}_{\mu} \frac{\partial q^{\mu}}{\partial x^{\mathrm{j}}}\right|_{\star}\right\}\left\{\left[\hat{p}_{\nu},\left.\frac{\partial q^{\nu}}{\partial x^{\mathrm{k}}}\right|_{\star}\right]_{+}+\left.\frac{\partial q^{\nu}}{\partial x^{\mathrm{k}}}\right|_{\star} \hat{p}_{\nu}\right\}
\end{aligned}
$$

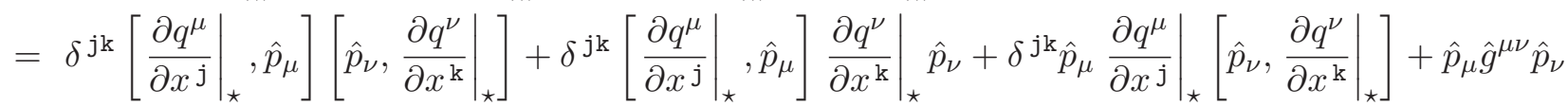

$$
\begin{aligned}
& =\hbar^{2} \delta^{\mathrm{jk}} \frac{\partial}{\partial q^{\mu}}\left(\frac{\partial q^{\mu}}{\partial x^{\mathrm{j}}}\right) \frac{\partial}{\partial q^{\nu}}\left(\frac{\partial q^{\nu}}{\partial x^{\mathrm{k}}}\right)_{\star}+i \hbar \delta^{\mathrm{jk}} \frac{\partial q^{\nu}}{\partial x^{\mathrm{k}}} \frac{\partial}{\partial q^{\mu}}\left(\frac{\partial q^{\mu}}{\partial x^{\mathrm{j}}}\right)_{\star} \hat{p}_{\nu}-i \hbar \delta^{\mathrm{jk}} \hat{p}_{\mu} \frac{\partial q^{\mu}}{\partial x^{\mathrm{j}}} \frac{\partial}{\partial q^{\nu}}\left(\frac{\partial q^{\nu}}{\partial x^{\mathrm{k}}}\right)_{\star}+\hat{p}_{\mu} \hat{g}^{\mu \nu} \hat{p}_{\nu} \text {, }
\end{aligned}
$$

o mesmo produto (4.22) é reduzido a

$$
\delta^{\mathrm{jk}} \hat{P}_{\mathrm{j}} \hat{P}_{\mathrm{k}}=\hat{p}_{\mu} \hat{g}^{\mu \nu} \hat{p}_{\nu}+\frac{i \hbar}{2} \delta^{\mathrm{jk}}\left[\frac{\partial q^{\nu}}{\partial x^{\mathrm{k}}} \frac{\partial}{\partial q^{\mu}}\left(\frac{\partial q^{\mu}}{\partial x^{\mathrm{j}}}\right)_{\star}, \hat{p}_{\nu}\right]+\frac{\hbar^{2}}{4} \delta^{\mathrm{jk}} \frac{\partial}{\partial q^{\mu}}\left(\frac{\partial q^{\mu}}{\partial x^{\mathrm{j}}}\right) \frac{\partial}{\partial q^{\nu}}\left(\frac{\partial q^{\nu}}{\partial x^{\mathrm{k}}}\right)_{\star} .
$$

Frente à particular observação que as derivações parciais entre a subvariedade $\mathcal{M}$ e o ambiente Euclideano se relacionam por

$$
\begin{aligned}
& \frac{\partial}{\partial x^{\mu}}\left(\frac{\partial q^{\mu}}{\partial x^{\mathrm{j}}}\right)_{\mathcal{Q}=0}=\frac{\partial}{\partial q^{\mu}}\left(\frac{\partial q^{\mu}}{\partial x^{\mathrm{j}}}\right)_{\mathcal{Q}=0}+\left.\Gamma_{\mu \alpha}^{\mu} \frac{\partial q^{\alpha}}{\partial x^{\mathrm{j}}}\right|_{\mathcal{Q}=0} \\
& \quad \Rightarrow \frac{\partial}{\partial q^{\mu}}\left(\frac{\partial q^{\mu}}{\partial x^{\mathrm{j}}}\right)_{\mathcal{Q}=0}=\frac{\partial}{\partial x^{\mu}}\left(\frac{\partial q^{\mu}}{\partial x^{\mathrm{j}}}\right)_{\mathcal{Q}=0}-\left.\Gamma_{\mu \alpha}^{\mu} \frac{\partial q^{\alpha}}{\partial x^{\mathrm{j}}}\right|_{\mathcal{Q}=0} \\
& \quad \Rightarrow \delta^{\mathrm{jk}} \frac{\partial q^{\nu}}{\partial x^{\mathrm{k}}} \frac{\partial}{\partial q^{\mu}}\left(\frac{\partial q^{\mu}}{\partial x^{\mathrm{j}}}\right)_{\mathcal{Q}=0}=\delta^{\mathrm{jk}} \frac{\partial q^{\nu}}{\partial x^{\mathrm{k}}} \frac{\partial}{\partial x^{\mu}}\left(\frac{\partial q^{\mu}}{\partial x^{\mathrm{j}}}\right)_{\mathcal{Q}=0}-\Gamma_{\mu \alpha}^{\mu} g^{\alpha \nu}, \quad \mathrm{e}
\end{aligned}
$$


diante da nulidade do primeiro termo à direita na última igualdade, visto a sua constituição por um produto de dois vetores normais entre si, podemos reexpressar (4.22) como

$$
\begin{aligned}
& \delta^{\mathrm{jk}} \hat{P}_{\mathrm{j}} \hat{P}_{\mathrm{k}}= \\
& =\hat{p}_{\mu} \hat{g}^{\mu \nu} \hat{p}_{\nu}+\left.\frac{\hbar^{2}}{2} \frac{\partial}{\partial q^{\nu}}\left(\Gamma_{\mu \alpha}^{\mu} g^{\alpha \nu}\right)\right|_{\star}+\frac{\hbar^{2}}{4} \delta^{\mathrm{jk}}\left(\frac{\partial}{\partial x^{\mu}} \frac{\partial q^{\mu}}{\partial x^{\mathrm{j}}}-\Gamma_{\mu \alpha}^{\mu} \frac{\partial q^{\alpha}}{\partial x^{\mathrm{j}}}\right)\left(\frac{\partial}{\partial x^{\nu}} \frac{\partial q^{\nu}}{\partial x^{\mathrm{k}}}-\Gamma_{\nu \beta}^{\nu} \frac{\partial q^{\beta}}{\partial x^{\mathrm{k}}}\right)_{\star} \\
& =\hat{p}_{\mu} \hat{g}^{\mu \nu} \hat{p}_{\nu}+\left.\frac{\hbar^{2}}{2} \frac{\partial}{\partial q^{\nu}}\left(\Gamma_{\mu \alpha}^{\mu} g^{\alpha \nu}\right)\right|_{\star}+\frac{\hbar^{2}}{4} \delta^{\mathrm{jk}} \frac{\partial}{\partial x^{\mu}}\left(\frac{\partial q^{\mu}}{\partial x^{\mathrm{j}}}\right) \frac{\partial}{\partial x^{\nu}}\left(\frac{\partial q^{\nu}}{\partial x^{\mathrm{k}}}\right)_{\star}+\frac{\hbar^{2}}{4} \hat{g}^{\mu \nu} \Gamma_{\alpha \mu}^{\alpha} \Gamma_{\beta \nu}^{\beta}
\end{aligned}
$$

mediante o uso da equação de Weingarten, haja vista que dela temos necessariamente

$$
\delta^{\mathrm{jk}} \Gamma_{\mu \alpha}^{\mu} \frac{\partial q^{\alpha}}{\partial x^{\mathrm{j}}} \frac{\partial}{\partial x^{\nu}}\left(\frac{\partial q^{\nu}}{\partial x^{\mathrm{k}}}\right)_{\mathcal{Q}=0}=0
$$

\section{A obtenção do Laplaciano}

Voltando as atenções para o primeiro termo desta igualdade, obtemos

$$
\begin{aligned}
& \mathrm{g}^{\frac{1}{4}} \hat{p}_{\mu} \hat{g}^{\mu \nu} \hat{p}_{\nu} \mathrm{g}^{\frac{1}{4}}= \\
& \quad=\left\{\hat{p}_{\mu} \mathrm{g}^{\frac{1}{4}}+\left[\mathrm{g}{ }^{\frac{1}{4}}, \hat{p}_{\mu}\right]\right\} \hat{g}^{\mu \nu}\left\{\mathrm{g}^{\frac{1}{4}} \hat{p}_{\nu}+\left[\hat{p}_{\nu}, \mathrm{g}^{\frac{1}{4}}\right]\right\}=\left(\hat{p}_{\mu} \mathrm{g}^{\frac{1}{4}}+i \hbar \frac{\partial \mathrm{g} \frac{1}{4}}{\partial q^{\mu}}\right) \hat{g}^{\mu \nu}\left(\mathrm{g}^{\frac{1}{4}} \hat{p}_{\nu}-i \hbar \frac{\partial \mathrm{g}^{\frac{1}{4}}}{\partial q^{\nu}}\right) \\
& \quad=\hat{p}_{\mu} \sqrt{\mathrm{g}} \hat{g}^{\mu \nu} \hat{p}_{\nu}-i \hbar \hat{p}_{\mu} \mathrm{g}^{\frac{1}{4}} \hat{g}^{\mu \nu} \frac{\partial \mathrm{g} \frac{1}{4}}{\partial q^{\nu}}+i \hbar \frac{\partial \mathrm{g} \frac{1}{4}}{\partial q^{\mu}} \hat{g}^{\mu \nu} \mathrm{g}^{\frac{1}{4}} \hat{p}_{\nu}+\hbar^{2} \frac{\partial \mathrm{g}^{\frac{1}{4}}}{\partial q^{\mu}} \hat{g}^{\mu \nu} \frac{\partial \mathrm{g} \frac{1}{4}}{\partial q^{\nu}},
\end{aligned}
$$

de onde segue, por uma simples consequência, que

$$
\begin{aligned}
\hat{p}_{\mu} \hat{g}^{\mu \nu} \hat{p}_{\nu}=\mathrm{g}^{-\frac{1}{4}}\left(\mathrm{~g}^{\frac{1}{4}} \hat{p}_{\mu} \hat{g}^{\mu \nu} \hat{p}_{\nu} \mathrm{g}^{\frac{1}{4}}\right) \mathrm{g}^{-\frac{1}{4}} & =\mathrm{g}^{-\frac{1}{4}} \hat{p}_{\mu} \sqrt{\mathrm{g}} \hat{g}^{\mu \nu} \hat{p}_{\nu} \mathrm{g}^{-\frac{1}{4}}-i \hbar \mathrm{g}^{-\frac{1}{4}} \hat{p}_{\mu} \mathrm{g}^{\frac{1}{4}} \hat{g}^{\mu \nu} \frac{\partial \mathrm{g}^{\frac{1}{4}}}{\partial q^{\nu}} \mathrm{g}^{-\frac{1}{4}} \\
& +i \hbar \mathrm{g}^{-\frac{1}{4}} \frac{\partial \mathrm{g}^{\frac{1}{4}}}{\partial q^{\mu}} \hat{g}^{\mu \nu} \mathrm{g}^{\frac{1}{4}} \hat{p}_{\nu} \mathrm{g}^{-\frac{1}{4}}+\hbar^{2} \mathrm{~g}^{-\frac{1}{4}} \frac{\partial \mathrm{g} \frac{1}{4}}{\partial q^{\mu}} \hat{g}^{\mu \nu} \frac{\partial \mathrm{g}^{\frac{1}{4}}}{\partial q^{\nu}} \mathrm{g}^{-\frac{1}{4}}
\end{aligned}
$$

Desta maneira, visto a observação feita em (C.6) trazer

$$
\mathrm{g}-\frac{1}{4} \frac{\partial \mathrm{g}^{\frac{1}{4}}}{\partial q^{\mu}}=\frac{1}{2} \Gamma_{\alpha \mu}^{\alpha}
$$

torna-se possível reescrever o mesmo produto como

$$
\hat{p}_{\mu} \hat{g}^{\mu \nu} \hat{p}_{\nu}=\mathrm{g}-\frac{1}{4} \hat{p}_{\mu} \sqrt{\mathrm{g}} \hat{g}^{\mu \nu} \hat{p}_{\nu} \mathrm{g}^{-\frac{1}{4}}-\frac{i \hbar}{2} \mathrm{~g}^{-\frac{1}{4}} \hat{p}_{\mu} \mathrm{g}^{\frac{1}{4}} \hat{g}^{\mu \nu} \Gamma_{\beta \nu}^{\beta}+\frac{i \hbar}{2} \hat{g}^{\mu \nu} \Gamma_{\alpha \mu}^{\alpha} \mathrm{g}{ }^{\frac{1}{4}} \hat{p}_{\nu} \mathrm{g}-\frac{1}{4}+\frac{\hbar^{2}}{4} \hat{g}^{\mu \nu} \Gamma_{\alpha \mu}^{\alpha} \Gamma_{\beta \nu}^{\beta}
$$


do qual, pela engenhosa observação de que

$$
\begin{aligned}
& \mathrm{g}^{-\frac{1}{4}} \hat{p}_{\mu}=\left[\mathrm{g}^{-\frac{1}{4}}, \hat{p}_{\mu}\right]+\hat{p}_{\mu} \mathrm{g}^{-\frac{1}{4}}=i \hbar \frac{\partial \mathrm{g}^{-\frac{1}{4}}}{\partial q^{\mu}}+\hat{p}_{\mu} \mathrm{g}^{-\frac{1}{4}} \Rightarrow \mathrm{g}^{-\frac{1}{4}} \hat{p}_{\mu} \mathrm{g}^{\frac{1}{4}}=-\frac{i \hbar}{2} \Gamma_{\alpha \mu}^{\alpha}+\hat{p}_{\mu}, \quad \mathrm{e} \\
& \hat{p}_{\nu} \mathrm{g}^{-\frac{1}{4}}=\left[\hat{p}_{\nu}, \mathrm{g}^{-\frac{1}{4}}\right]+\mathrm{g}^{-\frac{1}{4}} \hat{p}_{\nu}=-i \hbar \frac{\partial \mathrm{g}^{-\frac{1}{4}}}{\partial q^{\nu}}+\mathrm{g}^{-\frac{1}{4}} \hat{p}_{\nu} \Rightarrow \mathrm{g}^{\frac{1}{4}} \hat{p}_{\nu} \mathrm{g}^{-\frac{1}{4}}=\frac{i \hbar}{2} \Gamma_{\beta \nu}^{\beta}+\hat{p}_{\nu},
\end{aligned}
$$

vemos a redução de (4.25) a

$$
\begin{aligned}
\hat{p}_{\mu} \hat{g}^{\mu \nu} \hat{p}_{\nu} & =\mathrm{g}^{-\frac{1}{4}} \hat{p}_{\mu} \sqrt{\mathrm{g}} \hat{g}^{\mu \nu} \hat{p}_{\nu} \mathrm{g}^{-\frac{1}{4}}+\frac{i \hbar}{2}\left[\hat{g}^{\mu \nu} \Gamma_{\alpha \mu}^{\alpha}, \hat{p}_{\nu}\right]-\frac{\hbar^{2}}{4} \hat{g}^{\mu \nu} \Gamma_{\alpha \mu}^{\alpha} \Gamma_{\beta \nu}^{\beta} \\
& =\mathrm{g}^{-\frac{1}{4}} \hat{p}_{\mu} \sqrt{\mathrm{g}} \hat{g}^{\mu \nu} \hat{p}_{\nu} \mathrm{g}^{-\frac{1}{4}}-\frac{\hbar^{2}}{2} \frac{\partial}{\partial q^{\nu}}\left(\hat{g}^{\mu \nu} \Gamma_{\alpha \mu}^{\alpha}\right)-\frac{\hbar^{2}}{4} \hat{g}^{\mu \nu} \Gamma_{\alpha \mu}^{\alpha} \Gamma_{\beta \nu}^{\beta} .
\end{aligned}
$$

Logo, através da substituição deste particular resultado em (4.23), observamos que

$$
\delta^{\mathrm{jk}} \hat{P}_{\mathrm{j}} \hat{P}_{\mathrm{k}}=\Delta+\frac{\hbar^{2}}{4} \delta^{\mathrm{jk}} \frac{\partial}{\partial x^{\mu}}\left(\frac{\partial q^{\mu}}{\partial x^{\mathrm{j}}}\right) \frac{\partial}{\partial x^{\nu}}\left(\frac{\partial q^{\nu}}{\partial x^{\mathrm{k}}}\right)_{\star},
$$

sendo $\Delta=\mathrm{g}^{-\frac{1}{4}} \hat{p}_{\mu} \sqrt{\mathrm{g}} \hat{g}^{\mu \nu} \hat{p}_{\nu} \mathrm{g}^{-\frac{1}{4}}$ identificável com o mesmo operador de Laplace-Beltrami, associado à subvariedade $\mathcal{M}$, e obtido por DeWitt frente à canonicidade envolta em (1.4) ${ }^{13}$.

\subsubsection{Algumas considerações}

Pela adoção de $\mathcal{V}(\hat{q})=V(x(\hat{q}))$, percebemos que (4.26) permite retratar o Hamiltoniano (4.3) em termos dos canônicos $\hat{q}$ e $\hat{p}$, através de

$$
H(\hat{q}, \hat{p})=T(\hat{q}, \hat{p})+U(\hat{q})+\mathcal{V}(\hat{q})
$$

onde

$$
\begin{aligned}
& \hat{T}=T(\hat{q}, \hat{p})=\frac{1}{2 m} \Delta=\frac{1}{2 m} \mathrm{~g}^{-\frac{1}{4}} \hat{p}_{\mu} \sqrt{\mathrm{g}} \hat{g}^{\mu \nu} \hat{p}_{\nu} \mathrm{g}^{-\frac{1}{4}}, \mathrm{e} \\
& \hat{U}=U(\hat{q})=\frac{\hbar^{2}}{8 m} \delta^{j k} \frac{\partial}{\partial x^{\mu}}\left(\frac{\partial q^{\mu}}{\partial x^{\mathrm{j}}}\right) \frac{\partial}{\partial x^{\nu}}\left(\frac{\partial q^{\nu}}{\partial x^{\mathrm{k}}}\right)_{\star} .
\end{aligned}
$$

Em particular, com o efeito de $\Delta$ ser o laplaciano associado a $\mathcal{M}$, podemos identificar $\hat{T}$ não apenas como um operador estritamente cinético, mas exclusivamente dependente dos elementos intrínsecos a $\mathcal{M}$, assim como $\mathcal{V}(\hat{q})$.

\footnotetext{
${ }^{13}$ Vide, por exemplo, os comentários do Capítulo 1.
} 
Quanto ao operador $\hat{U}$ originário do mesmo produto (4.26) cabem algumas observações, sendo a primeira, e talvez mais nítida delas, atribuída à responsabilidade que $\hat{U}$ possui para com algum tipo de "correção quântica": trata-se de um resultado que surge pela tomada de $\mathcal{M}$ como uma subvariedade de outra Euclideana, e relacionado essencialmente à existência de produtos entre termos dependentes dos operadores $\hat{q}$ e $\hat{p}$ nos correspondentes quânticos de (2.6) que compõem necessariamente (4.26).

Desta forma por exemplo, notamos que a teoria quântica criada para o sistema clássico do Capítulo 3 não equivale à obtida por DeWitt, assim como a qualquer outra construída pela promoção dos parâmetros intrínsecos a $T^{*} \mathcal{M}$. Particularmente trata-se de uma diferença relacionada diretamente ao fato de que escolhas específicas foram, e sempre devem ser feitas diante da necessidade de um ordenamento de operadores, para a definição dos operadores quânticos únicos que associar-se-ão a cada um dos observáveis da teoria. Logo e a priori, "sempre" existirão diferenças, do ponto de vista quântico, entre todas as teorias físicas que podem ser criadas não apenas para a situação aqui em estudo, mas para um mesmo sistema físico de um modo geral ${ }^{14}$.

Entretanto devemos enfatizar, como uma segunda observação, que o resultado aqui obtido para $\hat{H}$ não deixa de ser interessante dado que, do comportamento de $\hat{T}$ e $\hat{\mathcal{V}}$ frente à parametrização intrínseca adotada, é notável que a covariância pode ser assegurada à teoria caso exista, por exemplo, algum fator diretamente relacionado ao mergulho isométrico remontando a um operador $\hat{U}$ identicamente nulo.

\subsection{A geometria do potencial quântico}

Mediante o fato da métrica Euclideana se expressar de maneira constante frente à parametrização $x=\left(x^{1}, \ldots, x^{\mathrm{D}+\mathrm{N}}\right)$, notamos que

$$
\frac{\partial Q^{\mathrm{r}}}{\partial x^{\mathrm{j}}} \frac{\partial}{\partial x^{\mathrm{a}}}\left(\frac{\partial x^{\mathrm{k}}}{\partial Q^{\mathrm{r}}}\right)+\frac{\partial x^{\mathrm{k}}}{\partial Q^{\mathrm{r}}} \frac{\partial}{\partial x^{\mathrm{a}}}\left(\frac{\partial Q^{\mathrm{r}}}{\partial x^{\mathrm{j}}}\right)=0
$$

Deste modo, como da mesma expressão vemos

$$
\begin{aligned}
& \frac{\partial}{\partial x^{\mu}}\left(\frac{\partial q^{\mu}}{\partial x^{\mathrm{j}}}\right)_{\star}=-\delta_{\mathrm{jk}} g^{\mu \alpha} \frac{\partial}{\partial x^{\mu}}\left(\frac{\partial x^{\mathrm{k}}}{\partial q^{\alpha}}\right)_{\star} \\
& \Rightarrow \delta^{\mathrm{jk}} \frac{\partial}{\partial x^{\mu}}\left(\frac{\partial q^{\mu}}{\partial x^{\mathrm{j}}}\right) \frac{\partial}{\partial x^{\nu}}\left(\frac{\partial q^{\nu}}{\partial x^{\mathrm{k}}}\right)_{\star}=\delta_{\mathrm{ab}}\left[g^{\mu \alpha} \frac{\partial}{\partial x^{\mu}}\left(\frac{\partial x^{\mathrm{a}}}{\partial q^{\alpha}}\right)\right]\left[g^{\nu \beta} \frac{\partial}{\partial x^{\nu}}\left(\frac{\partial x^{\mathrm{b}}}{\partial q^{\beta}}\right)\right]_{\star},
\end{aligned}
$$

\footnotetext{
${ }^{14}$ Discutiremos um pouco mais sobre isso ao final do próximo capítulo.
} 
se torna claro o desdobramento de (4.28) como

$$
U(\hat{q})=\frac{\hbar^{2}}{8} \delta_{\mathrm{ab}}\left[g^{\mu \alpha} \frac{\partial}{\partial x^{\mu}}\left(\frac{\partial x^{\mathrm{a}}}{\partial q^{\alpha}}\right)\right]\left[g^{\nu \beta} \frac{\partial}{\partial x^{\nu}}\left(\frac{\partial x^{\mathrm{b}}}{\partial q^{\beta}}\right)\right]_{\star},
$$

em plena coincidência para com a "correção quântica" obtida em [18] ${ }^{15}$.

Através da observação de que $\left\{\partial / \partial q^{1}, \ldots, \partial / \partial q^{\mathrm{D}}\right\}_{\mathcal{Q}=0}$ e $\left\{\partial / \partial \mathcal{Q}^{1}, \ldots, \partial / \partial \mathcal{Q}^{\mathrm{N}}\right\}_{\mathcal{Q}=0}$ são as respectivas bases para os planos tangente e normal a $\mathcal{M}$ num dos seus pontos, podemos escrever, por exemplo,

$$
\left.\frac{\partial^{2} x^{\mathrm{j}}}{\partial q^{\mu} \partial q^{\nu}}\right|_{\mathcal{Q}=0}=\left.a_{\mu \nu}^{\alpha} \frac{\partial x^{\mathrm{j}}}{\partial q^{\alpha}}\right|_{\mathcal{Q}=0}+\left.a_{\mu \nu}^{\mathrm{A}} \frac{\partial x^{\mathrm{j}}}{\partial \mathcal{Q}^{\mathrm{A}}}\right|_{\mathcal{Q}=0}
$$

onde $a_{\mu \nu}^{\mathrm{k}}$ são números reais. Logo, pelo uso de (2.8) e (C.2),

$$
a_{\mu \nu}^{\alpha}=\left.\frac{\partial q^{\alpha}}{\partial x^{j}} \frac{\partial^{2} x^{j}}{\partial q^{\mu} \partial q^{\nu}}\right|_{\mathcal{Q}=0}=\Gamma_{\mu \nu}^{\alpha}
$$

da qual obtemos, com o auxílio de (4.30), que

$$
\frac{\partial}{\partial x^{\mu}}\left(\frac{\partial x^{\mathrm{j}}}{\partial q^{\nu}}\right)_{\mathcal{Q}=0}=\left.\frac{\partial^{2} x^{\mathrm{j}}}{\partial q^{\mu} \partial q^{\nu}}\right|_{\mathcal{Q}=0}-\left.\quad \Gamma_{\mu \nu}^{\alpha} \frac{\partial x^{\mathrm{j}}}{\partial q^{\alpha}}\right|_{\mathcal{Q}=0}=\left.a_{\mu \nu}^{\mathrm{A}} \frac{\partial x^{\mathrm{j}}}{\partial \mathcal{Q}^{\mathrm{A}}}\right|_{\mathcal{Q}=0}
$$

visto a métrica (2.7), demonstrando efetivamente o caráter perpendicular dos vetores presentes em (4.29) que, por se dizer, permite-nos reformular a mesma expressão como

$$
U(\hat{q})=\frac{\hbar^{2}}{8 m} G_{\mathrm{AB}}\left(a_{\mu \alpha}^{\mathrm{A}} g^{\mu \alpha}\right)\left(a_{\nu \beta}^{\mathrm{B}} g^{\nu \beta}\right)_{\star} .
$$

Com o efeito das propriedades dos elementos pertencentes às bases supracitadas, notamos, de (4.31) por exemplo, que

$$
\begin{aligned}
a_{\mu \nu}^{\mathrm{A}} & =\frac{\partial \mathcal{Q}^{\mathrm{A}}}{\partial x^{\mathrm{j}}} \frac{\partial}{\partial x^{\mu}}\left(\frac{\partial x^{\mathrm{j}}}{\partial q^{\nu}}\right)_{\mathcal{Q}=0}=-\frac{\partial x^{\mathrm{j}}}{\partial q^{\nu}} \frac{\partial}{\partial x^{\mu}}\left(\frac{\partial \mathcal{Q}^{\mathrm{A}}}{\partial x^{\mathrm{j}}}\right)_{\mathcal{Q}=0} \\
& \Rightarrow a_{\mu \nu}^{\mathrm{A}} g^{\mu \nu}=-\delta^{\mathrm{jk}} \frac{\partial q^{\mu}}{\partial x^{\mathrm{j}}} \frac{\partial}{\partial x^{\mu}}\left(\frac{\partial \mathcal{Q}^{\mathrm{A}}}{\partial x^{\mathrm{k}}}\right)_{\mathcal{Q}=0},
\end{aligned}
$$

de onde identificamos o termo $a_{\mu \nu}^{\mathrm{A}} g^{\mu \nu}$ com o traço da aplicação $S^{\mathrm{A}}: T_{\mathrm{q}} \mathcal{M} \rightarrow T_{\mathrm{q}} \mathcal{M}$ definida em (2.12) ${ }^{16}$. Desta maneira se torna possível observar, através da expressão (2.13), a dependência

\footnotetext{
${ }^{15}$ Aliás, cabe notar que algumas das expressões que constam nesta seção também se fazem presentes em [18], face não apenas à coincidência do resultado de $\hat{H}$, mas principalmente ao objetivo de reinterpretar, se assim podemos dizer, o resultado (4.33) mostrado adiante.

${ }^{16}$ Observe que trata-se da mesma expressão mencionada como fruto da equação de Weingarten ao término do
} 
quadrática da "correção quântica" suprarreferida frente ao vetor de curvatura médio $\mathrm{H}$ da subvariedade $\mathcal{M}$, haja vista que

$$
\mathrm{H}^{2}=\frac{1}{\mathrm{D}^{2}} G_{\mathrm{AB}} \operatorname{Tr}\left(S^{\mathrm{A}}\right) \operatorname{Tr}\left(S^{\mathrm{B}}\right)
$$

segundo a métrica induzida no espaço normal a $\mathcal{M}$ num dos seus pontos. Assim, (4.32) se reexpressa como

$$
U(\hat{q})=\frac{\hbar^{2} \mathrm{D}^{2} \mathrm{H}^{2}}{8 m}
$$

Deste modo, e diferentemente do trabalho [18] onde também se constata a mesma expressão, devemos salientar, embasados nas observações tecidas ao término do Capítulo 2, que $\hat{H}$ tornar-se-á não apenas covariante frente à parametrização intrínseca, mas também liberto de qualquer tipo de "correção quântica", apenas quando $\mathcal{M}$ puder ser mergulhado minimamente como um todo no $\mathbb{R}^{\mathrm{D}+\mathrm{N}}$ : trata-se de algo perfeitamente possível, uma vez que existem ambientes Riemannianos submissos a este processo em específico, independentemente do processo de mergulho relacionado ser considerado geodésico ou não.

Conforme o observado no Capítulo 2, a particularidade de um mergulho geodésico é que todas as trajetórias $\alpha:(-\varepsilon, \varepsilon) \rightarrow \mathcal{M}$ realizadas por um sistema físico definido em $\mathcal{M}^{17}$, e caracterizadas como geodésicas nesta mesma subvariedade face ao Princípio da Mínima Ação $0^{18}$, também considerar-se-ão geodésicas no $\mathbb{R}^{\mathrm{D}+\mathrm{N}}$. Por consequência da nulidade da segunda forma fundamental nesta situação, uma vez que todo processo de mergulho geodésico é necessariamente mínimo, H será nulo e logo livrará o formalismo aqui presente de qualquer tipo de "correção quântica".

Entretanto, uma curva que se comporta como uma geodésica numa subvariedade $\mathcal{M}$ não necessariamente terá o mesmo comportamento na variedade onde $\mathcal{M}$ é mergulhada: no caso de um mergulho no $\mathbb{R}^{\mathrm{D+N}}$, isso acontecerá apenas quando a métrica associada à subvariedade independer da parametrização. Trata-se de um fato automaticamente relacionado à nulidade do tensor misto de curvatura (C.3), conforme se observou em [18], permitindo a $\mathcal{M}$ ser identificado como um ambiente plano, situação com a qual não lidamos haja vista que, se assim o fosse, não existiria qualquer problema relacionado ao ordenamento dos operadores que figuram no formalismo quântico Capítulo 2.

${ }^{17}$ Apenas por se dizer, $\varepsilon$ é um número real.

${ }^{18}$ Em verdade a $a c ̧ \tilde{a} o S$, definida neste caso por

$$
S=\int_{t_{1}}^{t_{2}} L(q(t), \dot{q}(t)) d t
$$

precisa apenas ser estacionária [7, 12] para a obtenção das equações de Euler-Lagrange que remontam às geodésicas $\alpha(t)=\left(q_{1}(t), \ldots, q_{\mathrm{D}}(t)\right)$, desde que mantidas fixas todas as coordenadas destas curvas quando em $t_{1}$ e $t_{2}$. 
Hamiltoniano. 


\section{Capítulo 5}

\section{Uma formulação alternativa}

\subsection{Considerações para a equivalência}

Antes de terminarmos este trabalho, se torna conveniente tecer alguns comentários adicionais referentes à construção de uma abordagem alternativa para a proposta encerrada nestas notas, sendo o primeiro deles diretamente relacionado ao formalismo clássico ${ }^{1}$.

Perante à constatação de que a superfície $\mathcal{M}$ figura como uma constante de movimento, quaisquer sejam as trajetórias $\alpha:(-\varepsilon, \varepsilon) \rightarrow \mathcal{M}$ realizadas pelo sistema físico, observamos que para elas vale

$$
\frac{d}{d t} f_{\mathrm{A}}(x)=\dot{x}^{\mathrm{j}} \frac{\partial f_{\mathrm{A}}}{\partial x^{\mathrm{j}}}=0
$$

Este particular resultado demonstra que o vetor velocidade, associado ao mesmo sistema, pertence necessariamente ao plano tangente $T_{\mathrm{q}} \mathcal{M}$ em cada um dos pontos q da subvariedade, conforme o também notado em (3.10).

Com efeito desta observação se torna possível, por exemplo, lidar com uma representação Lagrangiana alternativa $L^{\prime}: T \mathbb{R}^{\mathrm{D}+2 \mathrm{~N}} \rightarrow \mathbb{R}$ para o mesmo sistema físico, dada por

$$
L^{\prime}(x, \dot{x} ; \chi, \dot{\chi})=\frac{m}{2} \delta_{j \mathrm{k}} \dot{x}^{\mathrm{j}} \dot{x}^{\mathrm{k}}-V(x)-\chi^{\mathrm{A}} \dot{x}^{\mathrm{j}} \frac{\partial f_{\mathrm{A}}}{\partial x^{\mathrm{j}}}
$$

onde $\chi^{\mathrm{A}}$ são os multiplicadores de Lagrange utilizados para restringir o sistema à condição acima mencionada. Entretanto, para que essa representação seja válida, duas condições precisam ser impostas para torná-la equivalente a anterior.

(i) A primeira delas se associa diretamente à condição (5.1), uma vez que são infinitas as superfícies que a satisfazem. Deste modo, se torna vital impor $f_{\mathrm{A}}\left(x\left(t_{0}\right)\right)=0$ como condição

\footnotetext{
${ }^{1}$ Devemos frisar que esta seção talvez se constitua por uma resenha de algumas considerações feitas em [19].
} 
de contorno à equação (5.1), face à definição feita anteriormente para a subvariedade $\mathcal{M}^{2}$.

(ii) Já o segundo condicionamento se origina da observação que as diferentes representações Lagrangianas de um sistema físico, definido numa variedade $\mathcal{N}$ arbitrária, diferem apenas por um termo proporcional à derivada total em relação ao tempo de alguma função $h: \mathcal{N} \times(-\delta, \delta) \rightarrow \mathbb{R}$, sendo $\delta$ um número real [36]. Desta forma, como

$$
L^{\prime}-L=\lambda^{\mathrm{A}} f_{\mathrm{A}}(x)-\chi^{\mathrm{A}} \dot{x}^{\mathrm{j}} \frac{\partial f_{\mathrm{A}}}{\partial x^{\mathrm{j}}}
$$

se torna necessária a condição $\dot{\chi}^{\mathrm{A}}=-\lambda^{\mathrm{A}}$ para que toda a dinâmica procedente de $(5.2)$ equivalha a anterior.

\subsection{Consequências imediatas}

Através da suposição que (5.2) é válida para os nossos propósitos, iniciamos a construção do formalismo Hamiltoniano observando que os momentos conjugados às variáveis $x$ e $\chi$ se definem respectivamente por

$$
\Pi_{\mathrm{j}}=\frac{\partial L^{\prime}}{\partial \dot{x}^{\mathrm{j}}}=m \delta_{\mathrm{jk}} \dot{x}^{\mathrm{k}}-\chi^{\mathrm{A}} \frac{\partial f_{\mathrm{A}}}{\partial x^{\mathrm{j}}} \text { e } \rho_{\mathrm{A}}=\frac{\partial L^{\prime}}{\partial \dot{\chi}^{\mathrm{A}}}=0
$$

de onde notamos a presença das velocidades primariamente expressíveis

$$
\dot{x}^{\mathrm{j}}=v^{\mathrm{j}}(x, \Pi, \chi)=\frac{1}{m} \delta^{\mathrm{jk}}\left(\Pi_{\mathrm{k}}+\chi^{\mathrm{A}} \frac{\partial f_{\mathrm{A}}}{\partial x^{\mathrm{k}}}\right)
$$

assim como o primeiro vínculo do sistema, expresso por

$$
\Upsilon_{\mathrm{A}}(x, \chi ; \Pi, \rho)=\rho_{\mathrm{A}}=0
$$

A partir destas observações, a Hamiltoniana primária $H_{P}^{\prime}: T^{*} \mathbb{R}^{\mathrm{D}+2 \mathrm{~N}} \rightarrow \mathbb{R}$ se define como ${ }^{3}$

$$
H_{P}^{\prime}(x, \chi ; \Pi, \rho)=H^{\prime}(x, \chi ; \Pi, \rho)+\vartheta^{\mathrm{A}} \rho_{\mathrm{A}},
$$

\footnotetext{
${ }^{2}$ Vide as linhas iniciais do Capítulo 3, especialmente a expressão (3.1).

${ }^{3}$ Aqui $\vartheta=\dot{\chi}$ vale para que exista o necessário isomorfismo entre $T \mathbb{R}^{\mathrm{D}+2 \mathrm{~N}}$ e $T^{*} \mathbb{R}^{\mathrm{D}+2 \mathrm{~N}}$.
} 
sendo $H^{\prime}: T^{*} \mathbb{R}^{\mathrm{D}+2 \mathrm{~N}} \rightarrow \mathbb{R}$ a Hamiltoniana do sistema físico, dada por

$$
H^{\prime}(x, \chi ; \Pi, \rho)=\frac{1}{2 m} \delta^{j \mathrm{k}}\left(\Pi_{j}+\chi^{\mathrm{A}} \frac{\partial f_{\mathrm{A}}}{\partial x^{\mathrm{j}}}\right)\left(\Pi_{\mathrm{k}}+\chi^{\mathrm{B}} \frac{\partial f_{\mathrm{B}}}{\partial x^{\mathrm{k}}}\right)+V(x)
$$

Com o efeito da responsabilidade que (5.5) tem para com a definição da superfície regular onde o sistema físico se define em $T^{*} \mathbb{R}^{\mathrm{D}+2 \mathrm{~N}}$, a aplicação de condições de consistência sobre tais vínculos traz

$$
\begin{aligned}
\dot{\Upsilon}_{\mathrm{A}} & =\left\{\Upsilon_{\mathrm{A}}, H_{P}^{\prime}\right\}=0 \\
& =\frac{1}{m} \delta^{j \mathrm{k}} \frac{\partial f_{\mathrm{R}}}{\partial x^{\mathrm{j}}}\left(\Pi_{\mathrm{k}}\left\{\rho_{\mathrm{A}}, \chi^{\mathrm{R}}\right\}+\chi^{\mathrm{R}} \frac{\partial f_{\mathrm{S}}}{\partial x^{\mathrm{k}}}\left\{\rho_{\mathrm{A}}, \chi^{\mathrm{S}}\right\}\right)=-\frac{1}{m} \delta^{\mathrm{jk}} \frac{\partial f_{\mathrm{A}}}{\partial x^{\mathrm{j}}}\left(\Pi_{\mathrm{k}}+\chi^{\mathrm{B}} \frac{\partial f_{\mathrm{B}}}{\partial x^{\mathrm{k}}}\right)=0 .
\end{aligned}
$$

Deste modo, visto a independência desta igualdade em relação aos multiplicadores $\vartheta$, notamos que o sistema também se submete aos vínculos

$$
\Upsilon_{\mathrm{N}+\mathrm{A}}(x, \chi ; \Pi, \rho)=\delta^{\mathrm{jk}} \frac{\partial f_{\mathrm{A}}}{\partial x^{\mathrm{j}}}\left(\Pi_{\mathrm{k}}+\chi^{\mathrm{B}} \frac{\partial f_{\mathrm{B}}}{\partial x^{\mathrm{k}}}\right)=0
$$

funcionalmente independentes dos anteriores.

Condições análogas de consistência sobre os últimos vínculos também mostram

$$
\begin{aligned}
\dot{\Upsilon}_{\mathrm{N}+\mathrm{A}} & =\left\{\Upsilon_{\mathrm{N}+\mathrm{A}}, H_{P}^{\prime}\right\}=0 \\
& =\frac{1}{m} \delta^{j \mathrm{k}} \delta^{\mathrm{ab}} \Pi_{\mathrm{a}} \Pi_{\mathrm{k}}\left\{\frac{\partial f_{\mathrm{A}}}{\partial x^{\mathrm{j}}}, \Pi_{\mathrm{b}}\right\}+\frac{1}{m} \delta^{\mathrm{ab}} \chi^{\mathrm{B}} \Pi_{\mathrm{a}}\left\{A_{\mathrm{AB}}, \Pi_{\mathrm{b}}\right\} \\
& +\frac{1}{m} \delta^{\mathrm{jk}} \delta^{\mathrm{ab}} \chi^{\mathrm{C}} \Pi_{\mathrm{k}} \frac{\partial f_{\mathrm{C}}}{\partial x^{\mathrm{a}}}\left\{\frac{\partial f_{\mathrm{A}}}{\partial x^{\mathrm{j}}}, \Pi_{\mathrm{b}}\right\}+\frac{1}{m} \delta^{\mathrm{jk}} \delta^{\mathrm{ab}} \chi^{\mathrm{C}} \Pi_{\mathrm{b}} \frac{\partial f_{\mathrm{A}}}{\partial x^{\mathrm{j}}}\left\{\Pi_{\mathrm{k}}, \frac{\partial f_{\mathrm{C}}}{\partial x^{\mathrm{a}}}\right\} \\
& +\frac{1}{m} \delta^{\mathrm{ab}} \chi^{\mathrm{B}} \chi^{\mathrm{C}} \frac{\partial f_{\mathrm{C}}}{\partial x^{\mathrm{a}}}\left\{A_{\mathrm{AB}}, \Pi_{\mathrm{b}}\right\}+\frac{1}{2 m} \delta^{j \mathrm{k}} \chi^{\mathrm{C}} \chi^{\mathrm{D}} \frac{\partial f_{\mathrm{A}}}{\partial x^{\mathrm{j}}}\left\{\Pi_{\mathrm{k}}, A_{\mathrm{CD}}\right\} \\
& +\delta^{\mathrm{jk}} \frac{\partial f_{\mathrm{A}}}{\partial x^{\mathrm{j}}}\left\{\Pi_{\mathrm{k}}, V(x)\right\}+A_{\mathrm{AB}}\left\{\chi^{\mathrm{B}}, \rho_{\mathrm{C}}\right\} \vartheta^{\mathrm{C}}=0,
\end{aligned}
$$

de onde se evidencia a não submissão do sistema a qualquer outro vínculo, devido à aparição do multiplicador $\vartheta$. Em particular, com o uso da matriz $A$ definida anteriormente, temos

$$
\begin{aligned}
& A_{\mathrm{AB}} \vartheta^{\mathrm{B}}=A_{\mathrm{AB}}\left\{\chi^{\mathrm{B}}, \rho_{\mathrm{C}}\right\} \vartheta^{\mathrm{C}}= \\
& =-\frac{1}{m} \Pi^{\mathrm{j}} \Pi^{\mathrm{k}} \frac{\partial^{2} f_{\mathrm{A}}}{\partial x^{\mathrm{j}} \partial x^{\mathrm{k}}}-\frac{1}{m} \chi^{\mathrm{B}} \Pi^{\mathrm{j}} \frac{\partial A_{\mathrm{AB}}}{\partial x^{\mathrm{j}}}-\frac{1}{m} \delta^{\mathrm{ab}} \chi^{\mathrm{R}} \Pi^{\mathrm{j}} \frac{\partial f_{\mathrm{R}}}{\partial x^{\mathrm{a}}} \frac{\partial^{2} f_{\mathrm{A}}}{\partial x^{\mathrm{j}} \partial x^{\mathrm{b}}}+\delta^{\mathrm{jk}} \frac{\partial f_{\mathrm{A}}}{\partial x^{\mathrm{j}}} \frac{\partial V}{\partial x^{\mathrm{k}}} \\
& +\frac{1}{m} \delta^{\mathrm{jk}} \chi^{\mathrm{R}} \Pi^{\mathrm{a}} \frac{\partial f_{\mathrm{A}}}{\partial x^{\mathrm{j}}} \frac{\partial^{2} f_{\mathrm{R}}}{\partial x^{\mathrm{k}} \partial x^{\mathrm{a}}}-\frac{1}{m} \delta^{\mathrm{ab}} \chi^{\mathrm{B}} \chi^{\mathrm{R}} \frac{\partial f_{\mathrm{R}}}{\partial x^{\mathrm{a}}} \frac{\partial A_{\mathrm{AB}}}{\partial x^{\mathrm{b}}}+\frac{1}{2 m} \delta^{j \mathrm{k}} \chi^{\mathrm{R}} \chi^{\mathrm{S}} \frac{\partial f_{\mathrm{A}}}{\partial x^{\mathrm{j}}} \frac{\partial A_{\mathrm{RS}}}{\partial x^{\mathrm{k}}}
\end{aligned}
$$


da qual notamos

$$
\begin{aligned}
\vartheta^{\mathrm{A}} & =\bar{\vartheta}^{\mathrm{A}}(x, \chi ; \Pi, \rho)=\delta_{\mathrm{C}}^{\mathrm{A}} \vartheta^{\mathrm{C}}=A^{\mathrm{AB}} A_{\mathrm{BC}} \vartheta^{\mathrm{C}} \\
& =-\frac{1}{m} \Pi^{\mathrm{j}} \Pi^{\mathrm{k}} A^{\mathrm{AB}} \frac{\partial^{2} f_{\mathrm{B}}}{\partial x^{\mathrm{j}} \partial x^{\mathrm{k}}}-\frac{1}{m} \chi^{\mathrm{C}} \Pi^{\mathrm{j}} A^{\mathrm{AB}} \frac{\partial A_{\mathrm{BC}}}{\partial x^{\mathrm{j}}}-\frac{1}{m} \delta^{\mathrm{ab}} \chi^{\mathrm{R}} \Pi^{\mathrm{j}} A^{\mathrm{AB}} \frac{\partial f_{\mathrm{R}}}{\partial x^{\mathrm{a}}} \frac{\partial^{2} f_{\mathrm{B}}}{\partial x^{\mathrm{j}} \partial x^{\mathrm{b}}} \\
& +\delta^{\mathrm{jk}} A^{\mathrm{AB}} \frac{\partial f_{\mathrm{B}}}{\partial x^{\mathrm{j}}} \frac{\partial V}{\partial x^{\mathrm{k}}}+\frac{1}{m} \delta^{\mathrm{jk}} \chi^{\mathrm{R}} \Pi^{\mathrm{a}} A^{\mathrm{AB}} \frac{\partial f_{\mathrm{B}}}{\partial x^{\mathrm{j}}} \frac{\partial^{2} f_{\mathrm{R}}}{\partial x^{\mathrm{k}} \partial x^{\mathrm{a}}}-\frac{1}{m} \delta^{\mathrm{ab}} \chi^{\mathrm{C}} \chi^{\mathrm{R}} A^{\mathrm{AB}} \frac{\partial f_{\mathrm{R}}}{\partial x^{\mathrm{a}}} \frac{\partial A_{\mathrm{BC}}}{\partial x^{\mathrm{b}}} \\
& +\frac{1}{2 m} \delta^{\mathrm{jk}} \chi^{\mathrm{R}} \chi^{\mathrm{S}} A^{\mathrm{AB}} \frac{\partial f_{\mathrm{B}}}{\partial x^{\mathrm{j}}} \frac{\partial A_{\mathrm{RS}}}{\partial x^{\mathrm{k}}} .
\end{aligned}
$$

Pelo mesmo raciocínio, dado que (5.7) traz

$$
\delta^{j \mathrm{k}} \frac{\partial f_{\mathrm{A}}}{\partial x^{\mathrm{j}}}\left(\Pi_{\mathrm{k}}+\chi^{\mathrm{B}} \frac{\partial f_{\mathrm{B}}}{\partial x^{\mathrm{k}}}\right)=0 \Rightarrow \delta^{\mathrm{jk}} \frac{\partial f_{\mathrm{A}}}{\partial x^{\mathrm{j}}} \frac{\partial f_{\mathrm{B}}}{\partial x^{\mathrm{k}}} \chi^{\mathrm{B}}=A_{\mathrm{AB}} \chi^{\mathrm{B}}=-\frac{\partial f_{\mathrm{A}}}{\partial x^{\mathrm{j}}} \Pi^{\mathrm{j}}
$$

também se torna possível expressar cada $\chi^{\mathrm{A}}$ em termos das variáveis $x$ e $\Pi$. Explicitamente, isso é feito por

$$
\chi^{\mathrm{A}}=\bar{\chi}^{\mathrm{A}}(x, \Pi)=-A^{\mathrm{AB}} \frac{\partial f_{\mathrm{B}}}{\partial x^{\mathrm{j}}} \Pi^{\mathrm{j}}
$$

Com efeito desta particular observação, e visto (5.5) trazer $\rho_{\mathrm{A}}=\bar{\rho}_{\mathrm{A}}(x, \Pi)=0$, o par $(\chi, \rho)$ constituir-se-á, tão somente, por funções definidas num $T^{*} \mathbb{R}^{\mathrm{D}+\mathrm{N}}$ parametrizado pelas verdadeiras variáveis da nossa teoria clássica, no caso $z^{\prime}=(x, \Pi)$. Aliás, devido a estes mesmos resultados, também notamos que

$$
\vartheta^{\mathrm{A}}=\bar{\vartheta}^{\mathrm{A}}(x, \bar{\chi}(x, \Pi) ; \Pi, \bar{\rho}(x, \Pi))=\overline{\bar{\vartheta}}^{\mathrm{A}}(x, \Pi)
$$

Assim, através da substituição destas expressões em (5.6), a Hamiltoniana $\bar{H}^{\prime}: T^{*} \mathbb{R}^{\mathrm{D}+\mathrm{N}} \rightarrow \mathbb{R}$ para o sistema físico se torna

$$
\begin{aligned}
\bar{H}^{\prime}(x, \Pi) & =H^{\prime}(x, \bar{\chi}(x, \Pi) ; \Pi, \bar{\rho}(x, \Pi)) \\
& =\frac{1}{2 m} \delta^{\mathrm{jk}}\left(\delta_{j}^{\mathrm{b}}-\delta^{\mathrm{ba}} A^{\mathrm{AC}} \frac{\partial f_{\mathrm{A}}}{\partial x^{\mathrm{a}}} \frac{\partial f_{\mathrm{C}}}{\partial x^{\mathrm{j}}}\right) \Pi_{\mathrm{b}}\left(\delta_{\mathrm{k}}^{\mathrm{e}}-\delta^{\mathrm{ec}} A^{\mathrm{BY}} \frac{\partial f_{\mathrm{B}}}{\partial x^{\mathrm{c}}} \frac{\partial f_{\mathrm{Y}}}{\partial x^{\mathrm{k}}}\right) \Pi_{\mathrm{e}}+V(x),
\end{aligned}
$$

consequentemente reduzindo as equações de movimento associadas ao sistema por

$$
\dot{z}^{\prime}=\left\{z^{\prime}, \bar{H}^{\prime}\right\} \quad, \text { onde } z^{\prime}=(x, \Pi),
$$


dado a "extinção" dos vínculos da teoria, uma vez que eles se tornam identicamente nulos ${ }^{4}$.

\subsubsection{Observações pertinentes}

Algo nítido das considerações acima, em especial de (5.11), é que esse procedimento alternativo também não livra a formulação clássica dos produtos entre variáveis conjugadas e, por consequência, não é possível estabelecer uma teoria quântica para o mesmo sistema físico de forma unívoca, segundo a prescrição de Dirac.

Como se não bastasse, este formalismo também soa estranho numa primeira avaliação perante à "extinção" de todos os vínculos supracitada, uma vez que $z^{\prime}$ se constitui por parâmetros intrinsecamente relacionados ao $T^{*} \mathbb{R}^{\mathrm{D}+\mathbb{N}}$ e não à subvariedade $T^{*} \mathcal{M}$. No entanto, ao notarmos que a função $E_{\mathrm{k}}^{\mathrm{j}}: \mathbb{R}^{\mathrm{D}+\mathrm{N}} \rightarrow \mathbb{R}$, dada por

$$
E_{\mathrm{k}}^{\mathrm{j}}(x)=\delta_{\mathrm{k}}^{\mathrm{j}}-\delta^{\mathrm{j} 1} A^{\mathrm{AB}} \frac{\partial f_{\mathrm{A}}}{\partial x^{1}} \frac{\partial f_{\mathrm{B}}}{\partial x^{\mathrm{k}}},
$$

permite-nos reexpressar elegantemente a Hamiltoniana (5.11) como

$$
\bar{H}^{\prime}(x, \Pi)=\frac{1}{2 m} \delta^{\mathrm{jk}}\left(E_{\mathrm{j}}^{\mathrm{b}} \Pi_{\mathrm{b}}\right)\left(E_{\mathrm{k}}^{\mathrm{e}} \Pi_{\mathrm{e}}\right)+V(x)
$$

se torna clara uma certa similaridade com (3.7).

Uma vez que o particular uso de (5.14) revela-nos que

$$
\begin{aligned}
E_{\mathrm{k}}^{\mathrm{j}} E_{1}^{\mathrm{k}} & =\left(\delta_{\mathrm{k}}^{\mathrm{j}}-\delta^{\mathrm{ja}} A^{\mathrm{AB}} \frac{\partial f_{\mathrm{A}}}{\partial x^{\mathrm{a}}} \frac{\partial f_{\mathrm{B}}}{\partial x^{\mathrm{k}}}\right)\left(\delta_{1}^{\mathrm{k}}-\delta^{\mathrm{kb}} A^{\mathrm{CY}} \frac{\partial f_{\mathrm{C}}}{\partial x^{\mathrm{b}}} \frac{\partial f_{\mathrm{Y}}}{\partial x^{\mathrm{l}}}\right) \\
& =\delta_{1}^{\mathrm{j}}-\delta^{\mathrm{jb}} A^{\mathrm{CY}} \frac{\partial f_{\mathrm{C}}}{\partial x^{\mathrm{b}}} \frac{\partial f_{\mathrm{Y}}}{\partial x^{1}}-\left[\delta^{\mathrm{ja}} A^{\mathrm{AB}} \frac{\partial f_{\mathrm{A}}}{\partial x^{\mathrm{a}}} \frac{\partial f_{\mathrm{B}}}{\partial x^{1}}-\delta^{\mathrm{ja}} A^{\mathrm{AB}} \frac{\partial f_{\mathrm{A}}}{\partial x^{\mathrm{a}}}\left(\delta^{\mathrm{kb}} \frac{\partial f_{\mathrm{B}}}{\partial x^{\mathrm{k}}} \frac{\partial f_{\mathrm{C}}}{\partial x^{\mathrm{b}}}\right) A^{\mathrm{CY}} \frac{\partial f_{\mathrm{Y}}}{\partial x^{\mathrm{l}}}\right] \\
& =E_{1}^{\mathrm{j}}-\left(\delta^{\mathrm{ja}} A^{\mathrm{AB}} \frac{\partial f_{\mathrm{A}}}{\partial x^{\mathrm{a}}} \frac{\partial f_{\mathrm{B}}}{\partial x^{1}}-\delta^{\mathrm{ja}} A^{\mathrm{AB}} \frac{\partial f_{\mathrm{A}}}{\partial x^{\mathrm{a}}} \frac{\partial f_{\mathrm{Y}}}{\partial x^{\mathrm{l}}} \delta_{\mathrm{B}}^{\mathrm{Y}}\right)=E_{1}^{\mathrm{j}}, \\
E_{\mathrm{k}}^{\mathrm{j}} \frac{\partial f_{\mathrm{C}}}{\partial x^{\mathrm{j}}} & =\frac{\partial f_{\mathrm{C}}}{\partial x^{\mathrm{k}}}-\left(\delta^{\mathrm{ja}} \frac{\partial f_{\mathrm{C}}}{\partial x^{\mathrm{j}}} \frac{\partial f_{\mathrm{A}}}{\partial x^{\mathrm{a}}}\right) A^{\mathrm{AB}} \frac{\partial f_{\mathrm{B}}}{\partial x^{\mathrm{k}}}=\frac{\partial f_{\mathrm{C}}}{\partial x^{\mathrm{k}}}-\delta_{\mathrm{C}}^{\mathrm{B}} \frac{\partial f_{\mathrm{B}}}{\partial x^{\mathrm{k}}}=0,
\end{aligned}
$$

sendo $\partial f_{\mathrm{C}} / \partial x^{\mathrm{j}}$ um vetor normal à subvariedade (3.1) num dos seus pontos q, constatamos que $E_{\mathrm{k}}^{\mathrm{j}}$

\footnotetext{
${ }^{4}$ Em verdade, mesmo diante desta "extinção" de vínculos, devemos observar que a determinação específica feita em (5.10) revela-nos, com o auxílio de (3.3) e (5.3), que

$$
A_{\mathrm{AB}} \chi^{\mathrm{B}}=-P^{\mathrm{j}} \frac{\partial f_{\mathrm{A}}}{\partial x^{\mathrm{j}}}+A_{\mathrm{AB}} \chi^{\mathrm{B}} \Rightarrow P^{\mathrm{j}} \frac{\partial f_{\mathrm{A}}}{\partial x^{\mathrm{j}}}=0,
$$

resultado que se mostra compatível com (3.10). 
se comporta, em verdade, um projetor. Assim, como qualquer vetor definido nesta subvariedade se projeta apenas no subespaço $T_{\mathbf{q}} \mathcal{M}$, vemos que a Hamiltoniana (5.11) associada ao sistema é constituída apenas por um momento $P^{T}$ de componentes tangentes à subvariedade $\mathcal{M}$, dados por

$$
P_{\mathrm{j}}^{T}=E_{\mathrm{j}}^{\mathrm{k}} \Pi_{\mathrm{k}}
$$

logo D-dimensional. Algo interessante e relacionado a este particular resultado é que, apesar da "extinção" dos tradicionais vínculos que associar-se-iam ao formalismo aqui presente, uma restrição se faz presente na Hamiltoniana (5.11) frente à necessária composição desta apenas por momentos exclusivamente tangentes à superfície, analogamente a (3.7). Neste caso, podemos considerar que os vínculos não foram realmente extintos, haja vista que eles figuram implicitamente em (5.11).

Aliás, através das especiais substituições de (3.3) e (5.10) na igualdade (5.3), é imediato que

$$
\Pi_{j}=P_{j}-\chi^{\mathrm{A}} \frac{\partial f_{\mathrm{A}}}{\partial x^{\mathrm{j}}}=P_{j}+\delta^{\mathrm{k} 1} A^{\mathrm{AB}} \frac{\partial f_{\mathrm{B}}}{\partial x^{\mathrm{k}}} \frac{\partial f_{\mathrm{A}}}{\partial x^{\mathrm{j}}} \Pi_{1},
$$

de onde a simetricidade da matriz $A$ revela-nos

$$
P_{j}=\left(\delta_{j}^{1}-\delta^{1 \mathrm{k}} A^{\mathrm{AB}} \frac{\partial f_{\mathrm{A}}}{\partial x^{\mathrm{k}}} \frac{\partial f_{\mathrm{B}}}{\partial x^{\mathrm{j}}}\right) \Pi_{1}=E_{j}^{\mathrm{l}} \Pi_{1} .
$$

Ou seja, $P$ e $P^{T}$ se identificam como o mesmo vetor, implicando efetivamente na equivalência desta formulação Hamiltoniana com as duas mencionadas anteriormente apenas no caso desta respeitar aos dois condicionamentos listados no início deste capítulo. Por se dizer, diante deste fato e especialmente das relações clássicas de comutação, é perceptível, por exemplo, que

$$
\left\{x^{\mathrm{j}}, P_{\mathrm{k}}\right\}_{D\left(\Phi^{*}\right)}=E_{\mathrm{k}}^{\mathrm{j}}(x)=E_{\mathrm{k}}^{\mathrm{l}}(x)\left\{x^{\mathrm{j}}, \Pi_{1}\right\}=\left\{x^{\mathrm{j}}, P_{\mathrm{k}}^{T}\right\} .
$$

\subsubsection{Uma consideração adicional}

Entretanto devemos honestamente observar que, extraindo ambos condicionamentos supracitados, este formalismo Hamiltoniano alternativo não é muito consistente para com os nossos objetivos pois, conforme notamos dos seus resultados ${ }^{5}$ e, por exemplo em particular, da determinação da variável $\chi$ feita em (5.10), a única relação existente para como os vínculos da teoria apresentada

\footnotetext{
${ }^{5}$ Isso se deve ao fato de $L^{\prime}$ não ser auto suficiente para a determinação de toda a física relacionada ao sistema, diante da inexistência dos condicionamentos mencionados no início deste capítulo.
} 
no Capítulo 3 se relaciona ao fato de que

$$
P^{\mathrm{j}} \frac{\partial f_{\mathrm{A}}}{\partial x^{\mathrm{j}}}=0
$$

Deste modo, a única restrição que realmente existe, se refere apenas ao aspecto dos momentos conjugados às variáveis de posição serem necessariamente tangentes à superfície onde o sistema físico se define, não existindo, por exemplo, qualquer informação de como se descreve a própria superfície considerada. Isso permite-nos associar a última formulação apresentada com aquela de um sistema físico vinculado a uma superfície arbitrária, dada por

$$
f^{\mathrm{A}}(x)=c^{\mathrm{A}},
$$

onde as constantes $c^{\mathrm{A}}$ dizem respeito a quaisquer números reais, e não aos valores nulos especificamente exigidos pelo formalismo original ${ }^{6}$.

Para contornar este problema, devemos observar que outra possibilidade, que naturalmente surge com o efeito da mesma observação (ii) mencionada inicialmente, consiste em representar o sistema físico em pauta através da Lagrangiana $L^{\prime \prime}: T \mathbb{R}^{\mathrm{D}+3 \mathrm{~N}} \rightarrow \mathbb{R}$, dada por

$$
L^{\prime \prime}\left(x, \lambda^{\prime}, \chi^{\prime} ; \dot{x}, \dot{\lambda}^{\prime}, \dot{\chi}^{\prime}\right)=\frac{m}{2} \delta_{\mathrm{jk}} \dot{x}^{\mathrm{j}} \dot{x}^{\mathrm{k}}-V(x)-\lambda^{\prime \mathrm{A}} f_{\mathrm{A}}(x)-\chi^{\prime \mathrm{A}} \dot{x}^{\mathrm{j}} \frac{\partial f_{\mathrm{A}}}{\partial x^{\mathrm{j}}}
$$

visto que a própria (5.1) se define pela derivada total de uma função com respeito ao parâmetro temporal. Por consequência desta Lagrangiana, outro formalismo Hamiltoniano se ergue através de uma Hamiltoniana primária $H_{P}^{\prime \prime}: T^{*} \mathbb{R}^{\mathrm{D}+3 \mathrm{~N}} \rightarrow \mathbb{R}$ dada por ${ }^{7,8}$

$$
H_{P}^{\prime \prime}\left(x, \lambda^{\prime}, \chi^{\prime} ; \Pi^{\prime}, \pi^{\prime}, \rho^{\prime}\right)=H^{\prime \prime}\left(x, \lambda^{\prime} ; \Pi^{\prime}, \pi^{\prime}\right)+\lambda^{\prime \mathrm{A}} f_{\mathrm{A}}(x)+\xi^{\prime \mathrm{A}} \pi_{\mathrm{A}}^{\prime}+\vartheta^{\prime \mathrm{A}} \rho_{\mathrm{A}}^{\prime},
$$

com $H^{\prime \prime}: T^{*} \mathbb{R}^{\mathrm{D}+2 \mathrm{~N}} \rightarrow \mathbb{R}$ se referindo a

$$
H^{\prime \prime}\left(x, \lambda^{\prime} ; \Pi^{\prime}, \pi^{\prime}\right)=\frac{1}{2 m} \delta^{j \mathrm{k}} \Pi_{j}^{\prime} \Pi_{\mathrm{k}}^{\prime}+V(x)+\frac{1}{m} \delta^{\mathrm{jk}}\left(\Pi_{\mathrm{j}}^{\prime}+\frac{1}{2} \chi^{\prime \mathrm{A}} \frac{\partial f_{\mathrm{A}}}{\partial x^{\mathrm{j}}}\right) \chi^{\prime \mathrm{B}} \frac{\partial f_{\mathrm{B}}}{\partial x^{\mathrm{k}}}
$$

sendo os momentos $\Pi^{\prime}, \pi^{\prime}$ e $\rho^{\prime}$ conjugados aos respectivos parâmetros $x, \lambda^{\prime}$ e $\chi^{\prime}$, e dados, especi-

\footnotetext{
${ }^{6}$ Vide a expressão (3.1) presente no início do Capítulo 3.

${ }^{7}$ Devemos notar que a Hamiltoniana primária assim obtida também acorda com os comentários realizados ao longo do Apêndice A, especialmente os da Seção A.2.

${ }^{8}$ Mais uma vez convém frisar que $\xi^{\prime}=\dot{\lambda}^{\prime}$ e $\vartheta^{\prime}=\dot{\chi}^{\prime}$ para o necessário isomorfismo entre $T^{*} \mathbb{R}^{\mathrm{D}+3 \mathrm{~N}}$ e $T^{*} \mathbb{R}^{\mathrm{D}+3 \mathrm{~N}}$.
} 
ficamente nesta situação, por

$$
\Pi_{\mathrm{j}}^{\prime}=\frac{\partial L^{\prime \prime}}{\partial \dot{x}^{\mathrm{j}}}=m \delta_{j \mathrm{k}} \dot{x}^{\mathrm{k}}-\chi^{\prime \mathrm{A}} \frac{\partial f_{\mathrm{A}}}{\partial x^{\mathrm{j}}}, \pi_{\mathrm{A}}^{\prime}=\frac{\partial L^{\prime \prime}}{\partial \dot{\lambda}^{\prime \mathrm{A}}}=0, \rho_{\mathrm{A}}^{\prime}=\frac{\partial L^{\prime \prime}}{\partial \dot{\chi}^{\prime \mathrm{A}}}=0
$$

numa aparente coincidência, por exemplo, com as expressões (3.3) e (5.3) obtidas nos outros formalismos Hamiltonianos também presentes nestas notas.

Analogamente a todo o procedimento anterior, uma vez que

$$
\Psi_{\mathrm{A}}\left(x, \lambda^{\prime}, \chi^{\prime} ; \Pi^{\prime}, \pi^{\prime}, \rho^{\prime}\right)=\pi_{\mathrm{A}}^{\prime}=0 \text { e } \Psi_{\mathrm{N}+\mathrm{A}}\left(x, \lambda^{\prime}, \chi^{\prime} ; \Pi^{\prime}, \pi^{\prime}, \rho^{\prime}\right)=\rho_{\mathrm{A}}^{\prime}=0
$$

remontam aos vínculos primários desta nova teoria clássica, os quais definem uma parcela das componentes associadas à superfície onde o sistema físico se estabelece, é notável que deles seguem

$$
\begin{aligned}
\dot{\Psi}_{\mathrm{A}} & =\left\{\Psi_{\mathrm{A}}, H_{P}^{\prime \prime}\right\}=\left\{\pi_{\mathrm{A}}^{\prime}, \lambda^{\prime \mathrm{B}}\right\} f_{\mathrm{B}}(x)=-f_{\mathrm{A}}(x)=0 \\
\dot{\Psi}_{\mathrm{N}+\mathrm{A}} & =\left\{\Psi_{\mathrm{N}+\mathrm{A}}, H_{P}^{\prime \prime}\right\}=0 \\
& =\frac{1}{m} \delta^{j \mathrm{k}} \frac{\partial f_{\mathrm{B}}}{\partial x^{\mathrm{k}}}\left(\Pi_{\mathrm{j}}^{\prime}\left\{\rho_{\mathrm{A}}^{\prime}, \chi^{\prime \mathrm{B}}\right\}+\chi^{\prime \mathrm{B}} \frac{\partial f_{\mathrm{C}}}{\partial x^{\mathrm{j}}}\left\{\rho_{\mathrm{A}}^{\prime}, \chi^{\prime \mathrm{C}}\right\}\right)=-\frac{1}{m} \delta^{j \mathrm{k}} \frac{\partial f_{\mathrm{A}}}{\partial x^{\mathrm{j}}}\left(\Pi_{\mathrm{k}}^{\prime}+\chi^{\prime \mathrm{B}} \frac{\partial f_{\mathrm{B}}}{\partial x^{\mathrm{k}}}\right)
\end{aligned}
$$

o que permite observar a presença dos vínculos secundários

$$
\begin{gathered}
\Psi_{2 \mathrm{~N}+\mathrm{A}}\left(x, \lambda^{\prime}, \chi^{\prime} ; \Pi^{\prime}, \pi^{\prime}, \rho^{\prime}\right)=f_{\mathrm{A}}(x)=0 \\
\Psi_{3 \mathrm{~N}+\mathrm{A}}\left(x, \lambda^{\prime}, \chi^{\prime} ; \Pi^{\prime}, \pi^{\prime}, \rho^{\prime}\right)=\delta^{\mathrm{jk}} \frac{\partial f_{\mathrm{A}}}{\partial x^{\mathrm{j}}}\left(\Pi_{\mathrm{k}}^{\prime}+\chi^{\prime \mathrm{B}} \frac{\partial f_{\mathrm{B}}}{\partial x^{\mathrm{k}}}\right)=0
\end{gathered}
$$

visto explicações anteriores, sendo o primeiro deles idêntico a (3.9), enquanto o segundo se mostra apenas similar a $(5.7)^{9}$. Aliás de (5.21), se torna claro, por exemplo, que

$$
\begin{aligned}
\dot{\Psi}_{2 \mathrm{~N}+\mathrm{A}} & =\left\{\Psi_{2 \mathrm{~N}+\mathrm{A}}, H_{P}^{\prime \prime}\right\}=0 \\
& =\frac{1}{m} \delta^{\mathrm{jk}} \frac{\partial f_{\mathrm{A}}}{\partial x^{\mathrm{a}}}\left(\Pi_{\mathrm{j}}^{\prime}\left\{x^{\mathrm{a}}, \Pi_{\mathrm{k}}^{\prime}\right\}+\chi^{\prime \mathrm{B}} \frac{\partial f_{\mathrm{B}}}{\partial x^{\mathrm{k}}}\left\{x^{\mathrm{a}}, \Pi_{\mathrm{j}}^{\prime}\right\}\right)=\frac{1}{m} \delta^{\mathrm{jk}} \frac{\partial f_{\mathrm{A}}}{\partial x^{\mathrm{j}}}\left(\Pi_{\mathrm{k}}^{\prime}+\chi^{\prime \mathrm{B}} \frac{\partial f_{\mathrm{B}}}{\partial x^{\mathrm{k}}}\right) ;
\end{aligned}
$$

trata-se de um resultado que remonta ao mesmo segundo vínculo citado em (5.22). Portanto no que segue, as condições de consistência, relacionadas ao fato do conjunto total de vínculos $\Psi$ definir

\footnotetext{
${ }^{9}$ Devido a este fato, por exemplo, $\lambda$ pode ser interpretado mais uma vez como um multiplicador de Lagrange frente ao formalismo Hamiltoniano.
} 
a superfície sobre a qual o sistema físico deve se descrever, quando as expressões presentes neste conjunto serem necessariamente nulas, devem ser impostas apenas sobre $\Psi_{3 \mathrm{~N}+\mathrm{A}}$.

Dando sequência ao procedimento, temos

$$
\begin{aligned}
& \dot{\Psi}_{3 \mathrm{~N}+\mathrm{A}}=\left\{\Psi_{3 \mathrm{~N}+\mathrm{A}}, H_{P}^{\prime \prime}\right\}=0 \\
& =\frac{1}{m} \delta^{j \mathrm{k}} \delta^{\mathrm{ab}} \Pi_{\mathrm{a}}^{\prime} \Pi_{\mathrm{k}}^{\prime}\left\{\frac{\partial f_{\mathrm{A}}}{\partial x^{\mathrm{j}}}, \Pi_{\mathrm{b}}^{\prime}\right\}+\frac{1}{m} \delta^{j \mathrm{k}} \delta^{\mathrm{ab}} \chi^{\prime \mathrm{D}} \Pi_{\mathrm{k}}^{\prime} \frac{\partial f_{\mathrm{D}}}{\partial x^{\mathrm{b}}}\left\{\frac{\partial f_{\mathrm{A}}}{\partial x^{\mathrm{j}}}, \Pi_{\mathrm{a}}^{\prime}\right\}+\delta^{j \mathrm{k}} \frac{\partial f_{\mathrm{A}}}{\partial x^{\mathrm{j}}}\left\{\Pi_{\mathrm{k}}^{\prime}, V(x)\right\} \\
& +\frac{1}{m} \delta^{j \mathrm{k}} \delta^{\mathrm{ab}} \chi^{\prime \mathrm{D}} \Pi_{\mathrm{a}}^{\prime} \frac{\partial f_{\mathrm{A}}}{\partial x^{\mathrm{j}}}\left\{\Pi_{\mathrm{k}}^{\prime}, \frac{\partial f_{\mathrm{D}}}{\partial x^{\mathrm{b}}}\right\}+\delta^{j \mathrm{k}} \lambda^{\prime \mathrm{C}} \frac{\partial f_{\mathrm{A}}}{\partial x^{\mathrm{j}}}\left\{\Pi_{\mathrm{k}}^{\prime}, f_{\mathrm{C}}(x)\right\}+\frac{1}{2 m} \delta^{j \mathrm{k}} \chi^{\prime \mathrm{C}} \chi^{\prime \mathrm{D}} \frac{\partial f_{\mathrm{A}}}{\partial x^{\mathrm{j}}}\left\{\Pi_{\mathrm{k}}^{\prime}, A_{\mathrm{CD}}\right\} \\
& +\frac{1}{m} \delta^{\mathrm{ab}} \chi^{\prime \mathrm{B}} \Pi_{\mathrm{a}}^{\prime}\left\{A_{\mathrm{AB}}, \Pi_{\mathrm{b}}^{\prime}\right\}+\frac{1}{m} \delta^{\mathrm{ab}} \chi^{\prime \mathrm{B}} \chi^{\prime \mathrm{D}} \frac{\partial f_{\mathrm{D}}}{\partial x^{\mathrm{b}}}\left\{A_{\mathrm{AB}}, \Pi_{\mathrm{a}}^{\prime}\right\}+\vartheta^{\prime \mathrm{C}} A_{\mathrm{AB}}\left\{\chi^{\prime \mathrm{B}}, \rho^{\prime \mathrm{C}}\right\}=0
\end{aligned}
$$

com o auxílio da matriz $A$ definida no Capítulo 3 , e de onde vem

$$
\begin{aligned}
\dot{\Psi}_{3 \mathrm{~N}+\mathrm{A}} & =\frac{1}{m} \Pi^{\prime \mathrm{j}} \Pi^{\prime \mathrm{k}} \frac{\partial^{2} f_{\mathrm{A}}}{\partial x^{\mathrm{j}} \partial x^{\mathrm{k}}}+\frac{1}{m} \delta^{\mathrm{ab}} \chi^{\prime \mathrm{B}} \Pi^{\prime \mathrm{j}} \frac{\partial^{2} f_{\mathrm{A}}}{\partial x^{\mathrm{j}} \partial x^{\mathrm{a}}} \frac{\partial f_{\mathrm{B}}}{\partial x^{\mathrm{b}}}-\delta^{\mathrm{jk}} \frac{\partial f_{\mathrm{A}}}{\partial x^{\mathrm{j}}} \frac{\partial V}{\partial x^{\mathrm{k}}} \\
& -\frac{1}{m} \delta^{\mathrm{ab}} \chi^{\prime \mathrm{B}} \Pi^{\prime \mathrm{j}} \frac{\partial f_{\mathrm{A}}}{\partial x^{\mathrm{a}}} \frac{\partial^{2} f_{\mathrm{B}}}{\partial x^{\mathrm{j}} \partial x^{\mathrm{b}}}-\delta^{\mathrm{jk}} \lambda^{\prime \mathrm{B}} A_{\mathrm{AB}}-\frac{1}{2 m} \delta^{j \mathrm{k}} \chi^{\prime \mathrm{B}} \chi^{\prime \mathrm{C}} \frac{\partial f_{\mathrm{A}}}{\partial x^{\mathrm{j}}} \frac{\partial A_{\mathrm{BC}}}{\partial x^{\mathrm{k}}} \\
& +\frac{1}{m} \chi^{\prime \mathrm{B}} \Pi^{\prime \mathrm{j}} \frac{\partial A_{\mathrm{AB}}}{\partial x^{\mathrm{j}}}+\frac{1}{m} \delta^{j \mathrm{k}} \chi^{\prime \mathrm{B}} \chi^{\prime \mathrm{C}} \frac{\partial f_{\mathrm{C}}}{\partial x^{\mathrm{j}}} \frac{\partial A_{\mathrm{AB}}}{\partial x^{\mathrm{k}}}+\vartheta^{\prime \mathrm{B}} A_{\mathrm{AB}}=0 .
\end{aligned}
$$

Deste particular resultado é perceptível que a teoria aqui presente não é submissa a qualquer vínculo adicional, visto a aparição de $\vartheta^{\prime}$ nesta igualdade. Em particular, face à não singularidade de $A$, se torna possível obter uma expressão específica para as componentes $\vartheta^{\prime A}$ as quais, no caso, dão-se por

$$
\begin{aligned}
\vartheta^{\prime \mathrm{A}} & =\bar{\vartheta}^{\prime \mathrm{A}}\left(x, \lambda^{\prime}, \chi^{\prime} ; \Pi^{\prime}, \pi^{\prime}, \rho^{\prime}\right)=\lambda^{\prime \mathrm{A}}-\frac{1}{m} \Pi^{\prime \mathrm{j}} \Pi^{\prime \mathrm{k}} A^{\mathrm{AB}} \frac{\partial^{2} f_{\mathrm{B}}}{\partial x^{\mathrm{j}} \partial x^{\mathrm{k}}}-\frac{1}{m} \delta^{\mathrm{ab}} \chi^{\prime \mathrm{C}} \Pi^{\prime \mathrm{j}} A^{\mathrm{AB}} \frac{\partial^{2} f_{\mathrm{B}}}{\partial x^{\mathrm{j}} \partial x^{\mathrm{a}}} \frac{\partial f_{\mathrm{C}}}{\partial x^{\mathrm{b}}} \\
& +\delta^{\mathrm{jk}} A^{\mathrm{AB}} \frac{\partial f_{\mathrm{B}}}{\partial x^{\mathrm{j}}} \frac{\partial V}{\partial x^{\mathrm{k}}}+\frac{1}{m} \delta^{\mathrm{ab}} \chi^{\prime \mathrm{C}} \Pi^{\prime \mathrm{j}} A^{\mathrm{AB}} \frac{\partial f_{\mathrm{B}}}{\partial x^{\mathrm{a}}} \frac{\partial^{2} f_{\mathrm{C}}}{\partial x^{\mathrm{j}} \partial x^{\mathrm{b}}}+\frac{1}{2 m} \delta^{\mathrm{jk}} \chi^{\prime \mathrm{C}} \chi^{\prime \mathrm{D}} A^{\mathrm{AB}} \frac{\partial f_{\mathrm{B}}}{\partial x^{\mathrm{j}}} \frac{\partial A_{\mathrm{CD}}}{\partial x^{\mathrm{k}}} \\
& -\frac{1}{m} \chi^{\prime \mathrm{C}} \Pi^{\prime \mathrm{j}} A^{\mathrm{AB}} \frac{\partial A_{\mathrm{BC}}}{\partial x^{\mathrm{j}}}-\frac{1}{m} \delta^{\mathrm{jk}} \chi^{\prime \mathrm{C}} \chi^{\prime \mathrm{D}} A^{\mathrm{AB}} \frac{\partial f_{\mathrm{D}}}{\partial x^{\mathrm{j}}} \frac{\partial A_{\mathrm{BC}}}{\partial x^{\mathrm{k}}} .
\end{aligned}
$$

Através de uma similar argumentação, convém observar que a variável $\chi$ também fica determinada em função das demais, sob o uso de $\Psi_{3 \mathrm{~N}+\mathrm{A}}$, como

$$
\chi^{\prime \mathrm{A}}=\bar{\chi}^{\prime \mathrm{A}}\left(x, \Pi^{\prime}\right)=-A^{\mathrm{AB}} \frac{\partial f_{\mathrm{B}}}{\partial x^{\mathrm{j}}} \Pi^{\prime \mathrm{j}} .
$$

Por se dizer, esta expressão também se assemelha à mesma obtida em (5.10), e de onde a sua 
substituição em (5.23) mostra-nos que

$$
\vartheta^{\prime \mathrm{A}}=\bar{\vartheta}^{\prime \mathrm{A}}\left(x, \lambda^{\prime}, \bar{\chi}^{\prime}\left(x, \Pi^{\prime}\right) ; \Pi^{\prime}, \pi^{\prime}, \bar{\rho}^{\prime}\left(x, \Pi^{\prime}\right)\right)=\overline{\bar{\vartheta}}^{\prime \mathrm{A}}\left(x, \lambda^{\prime} ; \Pi^{\prime}, \pi^{\prime}\right)
$$

com o efeito de (5.3).

Algo também notável desta determinação de $\chi^{\prime}$ é que, por exemplo,

$$
\begin{aligned}
& \Pi_{j}^{\prime}+\frac{1}{2} \chi^{\prime \mathrm{A}} \frac{\partial f_{\mathrm{A}}}{\partial x^{\mathrm{j}}}=\Pi_{j}^{\prime}-\frac{1}{2} \Pi_{j}^{\prime}=\frac{1}{2} \Pi_{j}^{\prime} \\
& \quad \Rightarrow \delta^{j \mathrm{k}}\left(\Pi_{j}^{\prime}+\frac{1}{2} \chi^{\prime \mathrm{A}} \frac{\partial f_{\mathrm{A}}}{\partial x^{\mathrm{j}}}\right) \chi^{\prime \mathrm{B}} \frac{\partial f_{\mathrm{B}}}{\partial x^{\mathrm{k}}}=-\frac{1}{2} \delta^{j \mathrm{k}} \delta^{\mathrm{ba}} A^{\mathrm{BC}} \frac{\partial f_{\mathrm{B}}}{\partial x^{\mathrm{a}}} \frac{\partial f_{\mathrm{C}}}{\partial x^{\mathrm{k}}} \Pi_{j} \Pi_{\mathrm{b}}^{\prime}
\end{aligned}
$$

Este peculiar resultado, quando substituído em (5.19), permite-nos definir $\bar{H}^{\prime \prime}: T^{*} \mathbb{R}^{\mathrm{D}+\mathrm{N}} \rightarrow \mathbb{R}$ através de

$$
\begin{aligned}
\bar{H}^{\prime \prime}\left(x, \Pi^{\prime}\right) & =H^{\prime \prime}\left(x, \bar{\chi}\left(x, \Pi^{\prime}\right) ; \Pi^{\prime}, \bar{\rho}\left(x, \Pi^{\prime}\right)\right) \\
& =\frac{1}{2 m} \delta^{\mathrm{jk}}\left(\delta_{\mathrm{k}}^{\mathrm{b}}-\delta^{\mathrm{ba}} A^{\mathrm{BC}} \frac{\partial f_{\mathrm{B}}}{\partial x^{\mathrm{a}}} \frac{\partial f_{\mathrm{C}}}{\partial x^{\mathrm{k}}}\right) \Pi_{j}^{\prime} \Pi_{\mathrm{b}}^{\prime}+V(x),
\end{aligned}
$$

da qual, pelo uso de (5.14) e de suas propriedades projetivas, segue que ${ }^{10}$

$$
\bar{H}^{\prime \prime}\left(x, \Pi^{\prime}\right)=\frac{1}{2 m} \delta^{\mathrm{jk}}\left(E_{\mathrm{j}}^{\mathrm{b}} \Pi_{\mathrm{b}}^{\prime}\right)\left(E_{\mathrm{k}}^{\mathrm{e}} \Pi_{\mathrm{e}}^{\prime}\right)+V(x)=\bar{H}^{\prime}(x, \Pi)
$$

Ou seja, $\bar{H}^{\prime \prime}$ não apenas remete à Hamiltoniana física relacionada ao sistema em estudo, mas fundamentalmente revela-nos a equivalência desta teoria física com as anteriormente mencionadas. A diferença essencial que existe aqui se associa diretamente à não determinação de todos os multiplicadores de Lagrange, utilizados para a implementação dos vínculos que compõem $H_{P}^{\prime \prime}$, especificamente $\lambda^{\prime}$ e $\xi^{\prime}$ : neste caso, a mesma física associar-se-á ao sistema clássico, quaisquer sejam os valores assumidos não apenas por estes parâmetros não determinados, mas também por $\vartheta^{\prime}$, haja vista sua dependência explícita de $\lambda^{\prime}$.

Entretanto, independente de qualquer consideração adicional passível de aqui ser feita, devemos observar que, mais uma vez, deparamo-nos com uma teoria onde as funções associadas aos observáveis clássicos se impregnam por produtos entre os parâmetros conjugados. Logo se torna impossível realizar um processo de quantização liberto dos problemas relacionados à ambiguidade no ordenamento dos futuros operadores quânticos também sob este molde.

\footnotetext{
${ }^{10}$ Particularmente as mesmas observações que levam-nos a $(5.24)$, também dizem-nos que $\chi^{\prime}\left(x, \Pi{ }^{\prime}\right)=\chi(x, \Pi)$.
} 


\subsection{A construção de uma teoria quântica}

Diante das arbitrárias escolhas a priori possíveis para a quantização das teorias alternativas presentes neste capítulo, as quais se relacionam diretamente ao ordenamento de operadores supracitado $^{11}$, e apesar da evidente compatibilidade das suas formulações com a definida no Capítulo 3, convém notar que todas as teorias quânticas construídas a partir das abordagens singulares deste trabalho não necessariamente serão equivalentes: isso seguirá apenas se tomarmos escolhas específicas na determinação dos operadores quânticos autoadjuntos.

Como bem sabemos, apenas observações experimentais permitem identificar quais as teorias quânticas que, de fato, podem ser consideradas como físicas. Face a esta peculiar característica, e supondo que o processo de quantização realizado no Capítulo 4 satisfaz a este requisito, pode-se construir outra teoria física associada a qualquer uma das formulações do presente capítulo impondo, por exemplo, a sua equivalência quântica em relação a anterior como um princípio fundamental $[17]$.

Entretanto, no caso específico da última formulação apresentada, onde não puderam ser determinados todos os multiplicadores de Lagrange associados ao formalismo Hamiltoniano, devemos ter um cuidado adicional, uma vez que o processo geral de quantização de uma teoria Hamiltoniana singular é realizado, tão somente, sobre aquelas que se definem com o auxílio de um conjunto de vínculos exclusivos de segunda classe. Este cuidado figura como uma regra diretamente associada à plena determinação do ambiente clássico, no qual toda a dinâmica se descreve, conforme observamos no Apêndice A. Deste modo, antes de quantizar esta última teoria por exemplo, assim como qualquer outra também singular, é fundamental encontrar uma outra teoria, a ela equivalente, onde o conjunto de todos os vínculos seja composto exclusivamente pelos de segunda classe [7].

A particular tarefa de encontrar tais teorias físicas equivalentes, que supram a este peculiar condicionamento, em geral não é simples apesar do método utilizado para isso teoricamente o ser: tudo consiste em impor mais vínculos à teoria original através de uma fixação de calibre, que se responsabiliza por eliminar todas as arbitrariedades presentes na solução das equações de movimento das variáveis não envoltas na dinâmica do sistema físico; em verdade, são exatamente estas variáveis "não físicas" que se identificam com os multiplicadores de Lagrange, não especificamente resolvidos em termos das verdadeiras variáveis associadas ao sistema ${ }^{12}$. Logo, por uma mera consequência

\footnotetext{
${ }^{11}$ Vide as observações do Capítulo 4, especialmente das Seções 4.1 e 4.2 .

${ }^{12}$ No caso, estas equações de movimento se relacionam diretamente à não determinação de alguns multiplicadores em (A.7), devido à diferença existente entre o posto e a dimensão de $\Theta$, quando lidamos com uma teoria onde figuram vínculos de primeira classe. Maiores informações sobre este fato podem ser obtidas em [7], particularmente na sua
} 
desta fixação, todos os multiplicadores de Lagrange expressar-se-ão em termos dos parâmetros que descrevem o sistema físico, e automaticamente estará definida a teoria física almejada, composta unicamente por vínculos de segunda classe, e portanto passível de quantização pelo procedimento de Dirac [37].

Tomando como exemplo a última das teorias apresentadas neste capítulo, face à concordância dos seus resultados com aqueles originários das teorias clássicas anteriores, principalmente no que compete aos vínculos obtidos, uma possível saída para a quantização nos moldes supracitados consiste em adotar os resultados presentes em (3.14) e (3.15) para as respectivas variáveis não determinadas $\xi^{\prime}$ e $\lambda^{\prime}$, haja vista que elas claramente se exprimem em termos dos parâmetros $x$ e $\Pi$ mediante o uso, por exemplo, de (5.16) o que por consequência também determina $\vartheta^{\prime}$ em função dos mesmos parâmetros ${ }^{13}$.

Concomitantemente também podemos reexpressar $\bar{H}^{\prime \prime}$ de maneira a libertá-la de uma vinculação implícita sobre os momentos nela presentes, embora não libertando a teoria de um vínculo equivalente, e agora necessariamente explícito, que pertença ao desejado "conjunto de segunda classe", afinal o $\Psi^{*}=\left\{f_{\mathrm{A}}(x)=0\right\}$ com os únicos vínculos restantes à teoria, após todas estas determinações, é de primeira classe. No entanto cabe observar, visto o relacionamento (5.17), que uma teoria assim reformulada pode remontar exatamente à mesma já estruturada no Capítulo 3.

Apenas para finalizar todos os comentários, devemos frisar que as teorias clássica e quântica, responsáveis pela descrição de um sistema físico qualquer, são independentes por natureza, no sentido de que a "realidade quântica" deste não se descreve em verdade como a de um sistema clássico quantizado, a menos que considerações muito simplificadas sejam feitas, tais como a ignorância dos graus de liberdade intrinsecamente relacionados à abordagem quântica, para os quais não existem quaisquer análogos clássicos, assim como fizemos neste trabalho: trata-se apenas de uma postura adotada uma vez que, mesmo diante de tamanhas simplificações, podemos obter informações relevantes não apenas para construir uma teoria quântica que se enquadre como física, mas fundamentalmente para o conhecimento e a boa compreensão da natureza que cerca o mesmo

\footnotetext{
Seção 2.4 .

${ }^{13}$ Por se dizer, o fato de $\Pi$ e $\Pi^{\prime}$ serem identificados como iguais, mediante os argumentos da seção anterior, permite-nos constatar a igualdade entre (5.9) e (5.23) quando $\lambda=0$.

Indo um pouco mais além, também observamos dos quatro primeiros termos de (5.23), e essencialmente sob o uso
} de (5.10), de (5.14), assim como de suas propriedades projetivas, que

$$
\lambda^{\prime \mathrm{A}}-\frac{1}{m} \delta^{\mathrm{ab}} A^{\mathrm{AB}} \Pi^{\mathrm{j}} E_{\mathrm{b}}^{\mathrm{n}}(x) \Pi_{\mathrm{n}} \frac{\partial^{2} f_{\mathrm{B}}}{\partial x^{\mathrm{j}} \partial x^{\mathrm{a}}}+\delta^{\mathrm{jk}} A^{\mathrm{AB}} \frac{\partial f_{\mathrm{B}}}{\partial x^{\mathrm{j}}} \frac{\partial V}{\partial x^{\mathrm{k}}}=\lambda^{\prime \mathrm{A}}-A^{\mathrm{AB}}\left(\frac{1}{m} P^{\mathrm{j}} P^{\mathrm{k}} \frac{\partial^{2} f_{\mathrm{B}}}{\partial x^{\mathrm{j}} \partial x^{\mathrm{k}}}-\delta^{\mathrm{jk}} \frac{\partial f_{\mathrm{B}}}{\partial x^{\mathrm{j}}} \frac{\partial V}{\partial x^{\mathrm{k}}}\right) .
$$

Isso comprova que a adoção de (3.15) não apenas expressa $\vartheta^{\prime}$ em termos de um número menor de variáveis, como extingue os quatro termos supracitados de (5.23). 
sistema físico, frente o acordo destas considerações, embora simplificadas, com os resultados experimentalmente obtidos.

Olhando por este prisma, a proposta de Dirac compete na simples tentativa de remontar a uma nova teoria assumindo, como pré-requisito, a validade do príncipio da correspondência para com a teoria clássica entre outras coisas, apesar de não trazer qualquer informação consistente no que se refere ao seu estabelecimento unívoco: o que existe apenas é uma simples prescrição para um relacionamento entre as funções associadas aos observáveis da teoria clássica e os operadores quânticos, mas nada que aborde especificamente a determinação única dos últimos, envoltos nesta teoria quântica simplificada, frente aos requisitos de autoadjunticidade, conforme também foi mencionado em [6]. Assim, com o efeito desta lacuna, muitas teorias quânticas se erguem a priori para um mesmo sistema físico, cujo excesso poderá ser descartado apenas sob verificações experimentais, conforme já ressaltamos anteriormente. 


\section{Capítulo 6}

\section{Conclusões e comentários}

O objetivo deste trabalho foi apresentar essencialmente o problema relacionado ao estabelecimento de uma teoria quântica unívoca para um sistema físico com descrição clássica, especificamente constituído por uma partícula massiva em movimento não relativístico num espaço curvo $\mathcal{M}$ arbitrário, face aos produtos entre variáveis canonicamente conjugadas na formulação clássica Hamiltoniana.

Perante à constatação de que variedades Riemannianas podem ser mergulhadas isometricamente em espaços Euclideanos, apresentamos uma abordagem alternativa para o trato deste problema, intentando avaliar se a métrica Euclideana libertaria tal formulação Hamiltoniana dos produtos acima mencionados. Como já dissemos anteriormente, trata-se de um método alternativo intensamente estudado por diversos autores no início dos anos 90: aqui apenas detalhamos e analisamos a mesma questão, dando uma maior atenção ao aspecto geométrico envolvido neste tratamento.

Aliás, devido a essa preocupação geométrica, devemos salientar que diferentemente dos estudos anteriores, os quais dizem apenas que o mergulho isométrico de espaços Riemannianos ocorre nos Euclideanos com dimensões "suficientemente grandes" [21], mostramos que esta condição dimensional se relaciona diretamente com o teorema de Nash, haja vista que toda variedade Riemanniana D-dimensional possui um mergulho isométrico nos Euclideanos com D $(D+1)(3 D+11) / 2$ ou mais dimensões, salvo condições de metricidade.

Através da consideração de $\mathcal{M}$ como subvariedade de um espaço Euclideano com $\mathrm{N}$ dimensões extras satisfazendo por comodidade às condições de Nash, demonstramos que a teoria clássica obtida para o sistema físico definido na então subvariedade equivale a original, visto o processo de imersão isométrica induzir em $\mathcal{M}$ uma estrutura Riemanniana identificável com a da formulação original. Além disso, como essa abordagem leva necessariamente a uma teoria singular, também mostramos que o consequente uso de uma teoria de sistemas com vínculos não contribui para elimi- 
nar as ambiguidades presentes na descrição quântica do movimento não relativístico de partículas em espaços curvos, relacionadas à definição unívoca de operadores autoadjuntos, como já era conjecturado por Dirac [24] e conforme também se demonstrou nos artigos relacionados ao nosso trabalho, especificamente envoltos no mesmo tema [18, 19, 21].

Apesar da Hamiltoniana obtida com o artifício do mergulho ser livre dos produtos entre variáveis conjugadas, esses mesmos se fizeram presentes em outras funções relacionadas aos observáveis da teoria clássica, em especial na expressão de uma parte dos vínculos necessários à construção da subvariedade $T^{*} \mathcal{M}$. Portanto o processo de quantização se iniciou não apenas com base nas recomendações de Dirac, mas fazendo algumas escolhas relacionadas ao ordenamento de alguns operadores, visando o pleno estabelecimento da autoadjunticidade dos operadores almejados pela nova teoria física.

Entretanto, frente à existência de outro conjunto de parâmetros capazes de descrever o $T^{*} \mathbb{R}^{\mathrm{D}+\mathrm{N}}$, sendo parte destes intrínsecos a então subvariedade $T^{*} \mathcal{M}$, algumas observações relacionadas ao ordenamento de operadores puderam ser feitas. No caso do estabelecimento de um operador de momento autoadjunto e canonicamente conjugado à variável de posição, por exemplo, apesar de termos obtido uma expressão bem geral, pareceu-nos mais prudente optarmos por um ordenamento em particular que se mostrou equivalente com a escolha de Weyl para a mesma situação: mais especificamente, esta prudência se associou ao fato de não ser tão nítida a validade do princípio da correspondência. Com efeito desta escolha em particular, pudemos reexpressar o Hamiltoniano em termos de operadores canonicamente conjugados, observando a presença de um termo extra, nitidamente não covariante frente à parametrização intrínseca, sendo este termo responsável por algum tipo de "correção quântica" em decorrência do mergulho isométrico realizado.

Através da constatação de que se tratava de um termo diretamente proporcional ao quadrado do vetor de curvatura médio da subvariedade, fato também verificado em [17], mostramos que esta teoria em particular se torna covariante frente à parametrização se o espaço curvo, como um todo, puder ser mergulhado minimamente em outro Euclideano, uma vez que $\hat{H}$ expressar-se-á apenas em termos de parâmetros intrínsecos a $\mathcal{M}$ : trata-se de um aspecto não observado em quaisquer dos artigos relacionados que utilizamos como referência. Assim, perante à observação de que nem toda variedade Riemanniana se mergulha desta maneira, e como prospecto de pesquisas futuras, seria interessante investigar, por exemplo, não apenas quais variedades com interesse físico admitem um mergulho mínimo em ambientes Euclideanos, mas se, por ventura, também se existem, em outras situações de mergulho isométrico em variedades não Euclideanas, condições específicas para 
o ordenamento de operadores que permitem enxergar outros espaços curvos, onde o sistema físico também pode ser definido, como uma subvariedade mínima, intentando avaliar a covariância de teorias quânticas em situações mais gerais. Algo bem interessante, e de extrema utilidade também por exemplo, pode ser avaliar o comportamento de sistemas físicos definidos em espaços com torção sob este mesmo prisma: segundo [38], para um sistema formado por partículas sem spin e definido num destes espaços, toda a geometria a eles relacionada pode ser induzida pelo mergulho das trajetórias do sistema em espaços Euclideanos sem torção.

Conforme consta na literatura, e apenas a título de curiosidade, são poucas as subvariedades atualmente conhecidas com a propriedade de serem mínimas em todos os seus pontos, dentre as quais listamos planos, catenóides, helicóides, e as superfícies de Enneper e de Costa. Apesar de nada impedir a existência de outras subvariedades mínimas convém frisar, por exemplo, que a comprovação da última como uma delas levou alguns anos desde a sua conjecturação, feita pelo então doutorando Celso José da Costa ao longo dos seus trabalhos no IMPA, concluído em 1982: apenas com o auxílio de recursos computacionais, W. Meeks, um dos membros da banca examinadora do trabalho de Celso, e D. Hoffman conseguiram demonstrar matematicamente o caráter de subvariedade mínima apresentado por esta superfície. Por se dizer, tratou-se de uma das 25 descobertas científicas do ano 1985, segundo a Science News [39]. Ainda hoje muitas pesquisas se envolvem em investigações relacionadas às superfícies mínimas e, ao que vemos, o tema apresentado nestas notas figura como mais um deles.

Paralelo a tudo isso, também se torna interessante:

(i) estabelecer um relacionamento mais profundo entre os resultados que constam no trabalho aqui apresentado com o procedimento de quantização covariante de sistemas mecânicos apresentado em [8], visto a equivalência de alguns resultados, tais como a obtenção de quantizações covariantes, mediante o sempre necessário ordenamento de operadores, e os que especialmente se referem às ambiguidades relacionadas às distintas "correções quânticas", presentes em diversas teorias, as quais estão diretamente associadas às curvaturas; e

(ii) avaliar a existência dos operadores quânticos relacionados à situação aqui desenhada, uma vez que trata-se de um aspecto não claro, apesar do formalismo singular presente no Capítulo 3, por exemplo, remontar a uma teoria quântica liberta do condicionamento de irredutibilidade dos operadores quânticos, relacionados especificamente aos parâmetros usados na descrição do sistema físico. 
Antes de terminar todos os comentários, devemos enfatizar que o entendimento do que se resume nas teorias clássicas de sistemas vinculados e em suas consequentes quantizações é, de certo modo, essencial ao desenvolvimento de toda a Física teórica contemporânea. Como bom exemplo podemos citar o fato de todas as teorias envolvidas no estudo da física das partículas elementares serem teorias de calibre [16, 40], as quais configuram um caso especial das teorias Lagrangianas singulares, onde parte dos vínculos são de primeira classe [7]. 


\section{Apêndice A}

\section{Considerações Hamiltonianas}

\section{A.1 Algumas observações importantes}

Quando tomamos uma variedade Riemanniana $\mathcal{M}$ com $\mathrm{D}$ dimensões e duas funções $\mathcal{F}, \mathcal{G}$ : $T^{*} \mathcal{M} \rightarrow \mathbb{R}$, uma terceira se define automaticamente pelo uso dos chamados parênteses de Poisson expressos por

$$
\{\mathcal{F}, \mathcal{G}\}=\frac{\partial \mathcal{F}}{\partial q^{\mu}} \frac{\partial \mathcal{G}}{\partial p_{\mu}}-\frac{\partial \mathcal{F}}{\partial p_{\mu}} \frac{\partial \mathcal{G}}{\partial q^{\mu}}
$$

sendo $\omega=\left(\omega_{1}, \omega^{2}\right)=(q, p)$ uma 2-forma não degenerada com os parâmetros do fibrado cotangente $T^{*} \mathcal{M}$. Com o particular uso desta relação, observamos que

$$
\left\{\omega^{\mathrm{A}}, \omega^{\mathrm{B}}\right\}=\delta_{1}^{\mu} \delta_{2}^{\nu}-\delta_{2}^{\mu} \delta_{1}^{\nu}
$$

de onde se nota, da mesma (A.1), o desdobramento

$$
\{\mathcal{F}, \mathcal{G}\}=\frac{\partial \mathcal{F}}{\partial \omega_{1}^{\mu}} \frac{\partial \mathcal{G}}{\partial \omega_{\mu}^{2}}-\frac{\partial \mathcal{F}}{\partial \omega_{\mu}^{2}} \frac{\partial \mathcal{G}}{\partial \omega_{1}^{\mu}}=\frac{\partial \mathcal{F}}{\partial \omega^{\mathrm{A}}}\left\{\omega^{\mathrm{A}}, \omega^{\mathrm{B}}\right\} \frac{\partial \mathcal{G}}{\partial \omega^{\mathrm{B}}}
$$

Conforme consta na literatura, a descrição de qualquer sistema físico em $\mathcal{M}$ através de um par de variáveis canonicamente conjugadas $\omega$ ocorre pela adoção destes parênteses [36]. No caso, as equações de movimento associadas dão-se por

$$
\dot{\omega}=\{\omega, H\} \quad,
$$

onde $H: T^{*} \mathcal{M} \rightarrow \mathbb{R}$ é a Hamiltoniana representativa do sistema. Apesar desta particular parametrização, devemos enfatizar que a descrição de um sistema físico não se faz necessariamente por um único conjunto de variáveis canonicamente conjugadas: essa observação se respauda essencialmente 
no fato destes serem parâmetros relacionados à geometria intrínseca de $\mathcal{M}$, haja vista que variedades não se parametrizam por um único sistema de coordenadas ${ }^{1}$. Aliás, uma resposta específica sobre a existência de ao menos um conjunto de variáveis canonicamente conjugadas foi dada por Darboux, com a demonstração do teorema abaixo [41, 42].

Teorema 3 (de Darboux) Consideremos $\omega$ como uma 2-forma não degenerada numa variedade $\mathcal{N}$ com 2D dimensões. Então $\omega$ será simplética se, e somente se, em cada ponto de $\mathcal{N}$ existir uma vizinhança $\mathrm{U}$ onde ele se descreve por $\left(q^{1}, \ldots, q^{\mathrm{D}}, p_{1}, \ldots, p_{\mathrm{D}}\right)$ e tal que

$$
\left.\omega\right|_{U}=d q^{\mu} \wedge d p_{\mu}
$$

No caso aqui tratado, devemos identificar $\mathcal{N}$ com o fibrado cotangente $T^{*} \mathcal{M}$.

Dado a não unicidade suprarreferida, se torna possível tomar outra $\omega^{\prime}$ simplética para a descrição do mesmo sistema, trazendo equações de movimento expressas por

$$
\dot{\omega}^{\prime}=\left\{\omega^{\prime}, H^{\prime}\right\}
$$

com o uso de outra Hamiltoniana $H^{\prime}: T^{*} \mathcal{M} \rightarrow \mathbb{R}$, desde que exista fundamentalmente uma aplicação $\mathcal{A}: T^{*} \mathcal{M} \rightarrow T^{*} \mathcal{M}$ inversível de modo que a transformação canônica $\omega^{\prime}=\mathcal{A}(\omega)$ leve as equações de movimento (A.3) do sistema às expressões (A.4) e vice-versa. Por estas observações, é notável das mesmas $\mathcal{F}$ e $\mathcal{G}$ definidas acima que

$$
\frac{\partial \mathcal{F}}{\partial \omega^{\mathrm{A}}}=\frac{\partial \mathcal{F}}{\partial \omega^{\prime \mathrm{C}}} \frac{\partial \omega^{\prime \mathrm{C}}}{\partial \omega^{\mathrm{A}}}, \frac{\partial \mathcal{G}}{\partial \omega^{\mathrm{B}}}=\frac{\partial \mathcal{G}}{\partial \omega^{\prime \mathrm{D}}} \frac{\partial \omega^{\prime \mathrm{D}}}{\partial \omega^{\mathrm{B}}}
$$

as quais combinadas com (A.2) remontam a

$$
\{\mathcal{F}, \mathcal{G}\}=\frac{\partial \mathcal{F}}{\partial \omega^{\prime C}} \frac{\partial \omega^{\prime C}}{\partial \omega^{\mathrm{A}}}\left\{\omega^{\mathrm{A}}, \omega^{\mathrm{B}}\right\} \frac{\partial \omega^{\prime \mathrm{D}}}{\partial \omega^{\mathrm{B}}} \frac{\partial \mathcal{G}}{\partial \omega^{\prime \mathrm{D}}}=\frac{\partial \mathcal{F}}{\partial \omega^{\prime \mathrm{C}}}\left\{\omega^{\prime \mathrm{C}}, \omega^{\prime \mathrm{D}}\right\} \frac{\partial \mathcal{G}}{\partial \omega^{\prime \mathrm{D}}}
$$

revelando a invariância dos parênteses de Poisson por transformações canônicas.

\section{A.2 Sobre teorias com vínculos}

Entretanto, apesar do $T^{*} \mathcal{M}$ supracitado configurar uma variedade simplética, e portanto o teorema de Darboux assegurar, ao menos localmente, a existência de um $\omega$ com variáveis canonica-

\footnotetext{
${ }^{1}$ Vide a Seção 2.2, especialmente os últimos comentários da página 8.
} 
mente conjugadas para a descrição do sistema físico perante o formalismo clássico Hamiltoniano, a lida com estes parâmetros específicos nem sempre é conveniente. Por vezes, essa não conveniência se relaciona diretamente a eventual simplificação de cálculos, tornando mais cômoda a descrição de alguns sistemas em termos de um número maior de parâmetros que os intrinsecamente necessários; em outras são as próprias teorias físicas que ficam definidas originalmente em termos de um conjunto de parâmetros não independentes devido a algumas vantagens, tais como a exposição de eventuais simetrias que os sistemas possuem, ou à impossibilidade prática de expressar estas teorias em função dos parâmetros intrínsecos à variedade $\mathcal{M}$ onde os mesmos sistemas se definem.

Quando a adoção de um par $z=\left(z_{1}, z^{2}\right)$ de parâmetros não independentes ocorre, estamos em verdade considerando que o sistema físico se restringe necessariamente a algum subconjunto de uma variedade dimensionalmente maior $\mathcal{N}[3,23,43]$ : trata-se de uma restrição que, segundo o formalismo Hamiltoniano, se resume na existência de um conjunto $\Phi$, composto por funções $\Phi_{\mathrm{A}}: T^{*} \mathcal{N} \rightarrow \mathbb{R}$ que, quando restritas a $\Phi_{\mathrm{A}}=0$, se tornam as responsáveis diretas pela definição de $T^{*} \mathcal{M}$ como uma subvariedade de $T^{*} \mathcal{N}{ }^{2}$. Neste caso, o formalismo Hamiltoniano para um sistema físico fica construído com a determinação de uma Hamiltoniana total $H_{T}: T^{*} \mathcal{N} \rightarrow \mathbb{R}$ através de

$$
H_{T}=H+\lambda^{\mathrm{A}} \Phi_{\mathrm{A}}
$$

onde $\lambda^{\mathrm{A}}$ são os multiplicadores de Lagrange utilizados para a implementação do conjunto de vínculos $\Phi$ à Hamiltoniana $H: T^{*} \mathcal{M} \rightarrow \mathbb{R}$. Por consequência, a dinâmica do mesmo sistema se descreve por

$$
\dot{z}=\left.\left\{z, H_{T}\right\}\right|_{\Phi=0}
$$

\section{A.2.1 Informações relevantes}

De acordo com Dirac [24], uma função $\mathcal{F}: T^{*} \mathcal{N} \rightarrow \mathbb{R}$ será de primeira classe quando, na subvariedade definida por todos os vínculos supracitados, tivermos $\left\{\mathcal{F}, \Phi_{\mathrm{A}}\right\}=0$ para qualquer A; caso contrário $\mathcal{F}$ será de segunda classe. Com o efeito de cada vínculo figurar como um exemplo destas funções, a consideração da matriz quadrada $\Theta$ composta por elementos

$$
\Theta_{\mathrm{AB}}=\left\{\Phi_{\mathrm{A}}, \Phi_{\mathrm{B}}\right\},
$$

\footnotetext{
${ }^{2}$ Cabe frisar que $\Phi_{\mathrm{A}}^{\prime}: T^{*} \mathcal{N} \rightarrow \mathbb{R}$ devem ser sobrejetoras. Para maiores detalhes, vide o Capítulo 2, especialmente o exemplo apresentado na página 8 e a Seção 2.3 .
} 
permite-nos observar a necessária igualdade entre o seu posto e a quantidade de vínculos de segunda classe presentes em $\Phi$. Assim, caso $\Theta$ seja uma matriz singular, parte dos vínculos será obrigatoriamente de primeira classe [7, 44].

Pela adoção de um sistema físico onde figuram apenas vínculos de segunda classe, a consequente imposição de condições de regularidade sobre cada um deles, dadas especificamente por

$$
\dot{\Phi}_{\mathrm{B}}=\left\{\Phi_{\mathrm{B}}, H_{T}\right\}=\left\{\Phi_{\mathrm{B}}, H\right\}+\left\{\Phi_{\mathrm{B}}, \Phi_{\mathrm{A}}\right\} \lambda^{\mathrm{A}}=0,
$$

permitem notar que ${ }^{3}$

$$
\lambda^{\mathrm{A}}=-\Theta^{\mathrm{AB}}\left\{\Phi_{\mathrm{B}}, H\right\}
$$

Ou seja, todos os multiplicadores $\lambda^{\mathrm{A}}$ se expressam em função dos parâmetros de $T^{*} \mathcal{N}$ face à não singularidade da matriz $\Theta$, e portanto

$$
H_{T}=H-\Phi_{\mathrm{A}} \Theta^{\mathrm{AB}}\left\{\Phi_{\mathrm{B}}, H\right\} .
$$

De um modo geral, podemos dizer que a determinação de todos os multiplicadores de Lagrange presentes no formalismo Hamiltoniano, em termos dos parâmetros de $T^{*} \mathcal{N}$, se associa diretamente ao seguro estabelecimento do ambiente onde o sistema físico se define, uma vez que $\mathcal{M}$ deve ser vista como uma subvariedade de $\mathcal{N}^{4}$.

\section{A.3 Parênteses de Dirac e propriedades}

Perante à expressão obtida para a Hamiltoniana total, o uso dos parênteses de Dirac em (A.6) definidos por

$$
\{\mathcal{F}, \mathcal{G}\}_{D(\Phi)}=\{\mathcal{F}, \mathcal{G}\}-\left\{\mathcal{F}, \Phi_{j}\right\} \Theta^{\mathrm{jk}}\left\{\Phi_{\mathrm{k}}, \mathcal{G}\right\}
$$

onde $\mathcal{F}, \mathcal{G}: T^{*} \mathcal{N} \rightarrow \mathbb{R}$, permitem reexpressar as mesmas equações de movimento como

$$
\dot{z}=\{z, H\}_{D(\Phi)} \quad, \quad \text { com } \Phi=0
$$

Em particular, pela consideração das funções $\mathcal{F}, \mathcal{G}, \mathcal{K}: T^{*} \mathcal{N} \rightarrow \mathbb{R}$, de uma constante $\lambda$ ar-

\footnotetext{
${ }^{3}$ No caso, $\Theta_{A B}^{-1}=\Theta^{A B}$.

${ }^{4}$ Acreditamos que este aspecto se relaciona, por exemplo, ao fato do conjunto de vínculos de segunda classe possuir necessariamente um número par de elementos, diante da invertibilidade de $\Theta$. Neste caso, metade destes elementos ficam responsáveis pela particular definição de $\mathcal{M}$, enquanto a outra metade fixa um único plano tangente a cada ponto da então superfície regular $\mathcal{M}$.
} 
bitrária e um conjunto de vínculos $\Phi=\{\varphi, \psi\}$, composto pelos respectivos subconjuntos $\psi$ e $\varphi$ com vínculos exclusivos de primeira e segunda classe, podemos verificar que as seguintes propriedades se relacionam aos parênteses de Dirac:

(D.1)

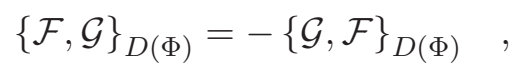

(D.2) $\{\mathcal{F}, \mathcal{G}+\lambda \mathcal{K}\}_{D(\Phi)}=\{\mathcal{F}, \mathcal{G}\}_{D(\Phi)}+\lambda\{\mathcal{F}, \mathcal{K}\}_{D(\Phi)} \quad$,

(D.3) $\{\mathcal{F}, \mathcal{G K}\}_{D(\Phi)}=\mathcal{G}\{\mathcal{F}, \mathcal{K}\}_{D(\Phi)}+\mathcal{K}\{\mathcal{F}, \mathcal{G}\}_{D(\Phi)} \quad$,

(D.4) $\left\{\mathcal{F},\{\mathcal{G}, \mathcal{K}\}_{D(\Phi)}\right\}_{D(\Phi)}+\left\{\mathcal{G},\{\mathcal{K}, \mathcal{F}\}_{D(\Phi)}\right\}_{D(\Phi)}+\left\{\mathcal{K},\{\mathcal{F}, \mathcal{G}\}_{D(\Phi)}\right\}_{D(\Phi)}=0$

(D.5) $\{\mathcal{F}, \mathcal{G}\}_{D(\Phi)}=\frac{\partial \mathcal{F}}{\partial z^{\mathrm{A}}}\left\{z^{\mathrm{A}}, z^{\mathrm{B}}\right\}_{D(\Phi)} \frac{\partial \mathcal{G}}{\partial z^{\mathrm{B}}} \quad \mathrm{e}$

(D.6) $\{\mathcal{F}, \mathcal{G}\}_{D(\Phi)}=\{\mathcal{F}, \mathcal{G}\}_{D(\varphi)}-\left\{\mathcal{F}, \psi_{j}\right\}_{D(\varphi)}\left(\{\psi, \psi\}_{D(\varphi)}\right)_{j \mathrm{k}}^{-1}\left\{\psi_{\mathrm{k}}, \mathcal{G}\right\}_{D(\varphi)}$.

Adicionalmente, com efeito da não unicidade das equações que definem a subvariedade $T^{*} \mathcal{M}$, podemos tomar outro conjunto de vínculos $\Psi$, dado por funções $\Psi_{\mathrm{A}}: T^{*} \mathcal{N} \rightarrow \mathbb{R}$ restritas a $\Psi_{\mathrm{A}}=0$, equivalente aos suprarreferidos, tornando possível observar que ${ }^{5}$

(D.7) $\left\{\mathcal{F}, \Phi_{j}\right\}_{D(\varphi)}=0$

(D.8) $\{\mathcal{F},\{\Phi\}\}_{D(\varphi)}=\{\Phi\} \quad$ e

(D.9) $\{\mathcal{F}, \mathcal{G}\}_{D(\Phi)}=\{\mathcal{F}, \mathcal{G}\}_{D(\Psi)}+\{\Phi\}$,

como propriedades adicionais, onde $\{\Phi\}$ denota uma combinação linear de vínculos do conjunto $\Phi$. Aliás de acordo com estas observações, e principalmente com aquelas que se relacionam à definição de $T^{*} \mathcal{M}$ como uma subvariedade, as equações (A.9) se expressam por meio do último conjunto de vínculos através de

$$
\dot{z}=\{z, H\}_{D(\Psi)} \quad, \operatorname{com} \Psi(z)=0
$$

Por consequência das propriedades listadas acima também notamos que a consideração de um conjunto de variáveis $z^{\prime}$, obtido através de uma transformação canônica em $z$, reexpressam as mesmas (A.9) como

$$
\dot{z}^{\prime}=\left\{z^{\prime}, H^{\prime}\right\}_{D\left(\Phi^{\prime}\right)} \quad, \operatorname{com} \Phi^{\prime}\left(z^{\prime}\right)=0
$$

\footnotetext{
${ }^{5}$ Esta equivalência se relaciona diretamente ao fato de $T^{*} \mathcal{M}$ também se definir pelo novo conjunto de vínculos. Apenas por questões de reforço, devemos frisar a sobrejetividade de $\Psi_{\mathrm{A}}^{\prime}: T^{*} \mathcal{N} \rightarrow \mathbb{R}$.
} 
dado que os parênteses de Dirac se constituem pelos de Poisson, e portanto são invariantes pela mesma transformação. Neste caso em particular, as funções $H^{\prime}, \Phi^{\prime}: T^{*} \mathcal{N} \rightarrow \mathbb{R}$ devem necessariamente satisfazer às relações

$$
H^{\prime}\left(z^{\prime}\right)=H(z) \quad, \Phi^{\prime}\left(z^{\prime}\right)=\Phi(z)
$$

\section{A.4 Comentários adicionais}

Conforme salientamos anteriormente, o teorema de Darboux assegura que, ao menos localmente, qualquer sistema clássico Hamiltoniano se descreve em termos de parâmetros intrínsecos ao fibrado cotangente onde ele se define, os quais constituem um conjunto natural de variáveis canonicamente conjugadas $\omega$. No caso específico de um sistema originalmente descrito por uma teoria com vínculos exclusivos de segunda classe, está provado que a obtenção dos parâmetros canônicos assegurados por Darboux ocorre por meio de uma transformação canônica sobre o par $z$, constituído pelas variáveis não independentes da teoria original [7]. Assim, face à consequente "extinção" dos vínculos por essa transformação em específico ${ }^{6}$, as equações (A.10) e (A.11) permitem descrever a dinâmica do mesmo sistema por

$$
\dot{\omega}=\left\{\omega, H_{F}\right\}
$$

sendo $H_{F}: T^{*} \mathcal{M} \rightarrow \mathbb{R}$ a sua Hamiltoniana. Porém, devido a não unicidade na determinação de $\omega$, cabe ressaltar, mais uma vez que, todas as 2-formas simpléticas satisfazendo a (A.12) se relacionam por transformações canônicas.

Pra encerrar esta discussão, devemos enfatizar uma importante propriedade apresentada por um sistema Hamiltoniano com parte dos vínculos de segunda classe: um subconjunto completo de variáveis conjugadas da teoria se expressa em função das demais. Trata-se de uma mera consequência devido a um subconjunto $\Phi_{*}$ dos vínculos de segunda classe se compor, em verdade, por equações que permitem essa plena determinação.

Explicitamente, ao tomarmos um sistema definido em alguma variedade $T^{*} \mathcal{N}$ parametrizada por $z=\left(z^{*}, z_{*}\right)$ e condicionado aos vínculos $\Phi=\left\{\Phi^{*}, \Phi_{*}\right\}$, se observa a existência de uma aplicação $\mathcal{Z}: T^{*} \mathcal{N}^{\prime} \rightarrow T^{*} \mathcal{N}^{\prime \prime}$ definida por

$$
\Phi_{*}(z)=0 \Rightarrow z_{*}=\mathcal{Z}\left(z^{*}\right)
$$

\footnotetext{
${ }^{6}$ No caso, o conjunto $\Omega$ com os vínculos equivalentes e originários desta particular transformação se torna identicamente nulo, quaisquer sejam os valores assumidos pelos novos parâmetros.
} 
sendo $T^{*} \mathcal{N}^{\prime}$ e $T^{*} \mathcal{N}^{\prime \prime}$ ambientes satisfazendo a $T^{*} \mathcal{N}^{\prime} \cup T^{*} \mathcal{N}^{\prime \prime}=T^{*} \mathcal{N}$. Logo a dinâmica do sistema em questão fica descrita por

$$
\dot{z}^{*}=\left.\left\{z^{*}, \bar{H}\right\}_{D\left(\Phi^{*}\right)}\right|_{\Phi^{*}=0},
$$

onde $\bar{H}: T^{*} \mathcal{N}^{\prime} \rightarrow \mathbb{R}$ é a Hamiltoniana a ele associada, uma vez que $\Phi^{*}\left(z^{*}\right)=0$ são os únicos vínculos essenciais à definição da subvariedade $T^{*} \mathcal{N}^{\prime} \subset T^{*} \mathcal{N}$ onde o mesmo sistema se descreve. Nos casos onde o sistema se submete apenas ao subconjunto de vínculos especiais que remetem a (A.13), é imediato que

$$
\operatorname{dim} \Phi=\operatorname{dim} \Phi_{*}=\operatorname{dim}\left(T^{*} \mathcal{N}^{\prime \prime}\right)
$$

tornando possível identificar $z^{*}$ como uma das $\omega$ simpléticas existentes para a mesma teoria. 


\section{Apêndice B}

\section{Uma observação importante}

O objetivo deste Apêndice é apresentar um teorema importante referente ao exemplo de superfície mencionada no Capítulo 2, mais precisamente na página 8. Por se dizer, as linhas abaixo são uma mera adaptação de [22].

Teorema 4 Sejam $\mathrm{U} \subset \mathbb{R}^{\mathrm{D}+\mathrm{N}}$ um aberto, $f: \mathrm{U} \rightarrow \mathbb{R}^{\mathrm{N}}$ uma aplicação de classe $C^{\mathrm{k}}$ e tomemos $c \in \mathbb{R}^{\mathrm{N}}$. Considerando o conjunto

$$
\mathcal{S}=\left\{\mathrm{q} \in \mathrm{U}: f(\mathrm{q})=c \text { e e } f^{\prime}(\mathrm{q}): \mathbb{R}^{\mathrm{D}+\mathrm{N}} \rightarrow \mathbb{R}^{\mathrm{D}} \text { é sobrejetora }\right\}
$$

aberto em $f^{-1}(c)$, então:

(A.1) supondo que $\mathcal{S}$ é não vazio, logo $\mathcal{S}$ é uma superfície de dimensão $\mathrm{D}$ e classe $C^{\mathrm{k}}$ do $\mathrm{U} \subset \mathbb{R}^{\mathrm{D}+\mathrm{N}}$;

(A.2) $T_{\mathrm{q}} \mathcal{S}=\operatorname{ker} f^{\prime}(\mathrm{q})$, para todo $\mathrm{q} \in \mathcal{S}$.

Demonstração. Tomemos q como um ponto de $\mathcal{S}$. De acordo com o teorema das funções implícitas [25], existem

(i) uma decomposição do $\mathbb{R}^{\mathrm{D}+\mathrm{N}}$ como $\mathbb{R}^{\mathrm{D}} \oplus \mathbb{R}^{\mathrm{N}}$, com $\mathrm{q}=\left(\mathrm{q}^{1}, \mathrm{q}^{2}\right)$ onde $\mathrm{q}^{1}$ e $\mathrm{q}$ pertencem às respectivas vizinhanças $\mathrm{U}_{1} \subset \mathbb{R}^{\mathrm{D}}$ e $\mathrm{U}_{2} \subset \mathbb{R}^{\mathrm{D}+\mathrm{N}}$, e

(ii) uma aplicação $\xi: \mathrm{U}_{1} \rightarrow \mathbb{R}^{\mathrm{D}}$ de classe $C^{\mathrm{k}}$, tal que $\mathcal{G}(\xi)=\mathrm{U}_{2} \cap f^{-1}(c)$.

Deste modo, a aplicação $\phi: \mathrm{U}_{1} \rightarrow \mathrm{U}_{2} \cap f^{-1}(c)$, dada por

$$
\phi\left(x^{1}\right)=\left(x^{1}, \xi\left(x^{1}\right)\right)
$$


serve como uma parametrização, também de classe $C^{\mathrm{k}}$, para uma vizinhança aberta de $\mathrm{q} \in f^{-1}(c)$. Logo, como o fato de $f^{\prime}(\mathrm{q})$ ser sobrejetora implica que o conjunto dos pontos $\mathrm{q} \in \mathbb{R}^{\mathrm{D}+\mathrm{N}}$ é aberto, (A.1) fica demonstrado uma vez que $\mathrm{U}_{2} \cap f^{-1}(c) \subset \mathcal{S}$.

Já o item (A.2) segue por tomarmos uma curva $\lambda:(-\varepsilon, \varepsilon) \rightarrow \mathcal{S}$, sendo $\varepsilon$ um número real, com $\lambda(0)=\mathrm{q}$ e $\lambda^{\prime}(0)=v$, onde $v \in T_{\mathrm{q}} \mathcal{S}$. Neste caso,

$$
f^{\prime}(\mathbf{q}) \cdot v=f^{\prime}(\lambda(0)) \cdot \lambda^{\prime}(0)=(f \circ \lambda)^{\prime}(0)=0
$$

haja vista que $f \circ \lambda$ possui valor constante, implicando em $v \in \operatorname{ker} f^{\prime}(\mathrm{q})$. Assim, como $T_{\mathrm{q}} \mathcal{S}$ e ker $f^{\prime}(\mathrm{q})$ são subespaços do $\mathbb{R}^{\mathrm{D}+\mathrm{N}}$, ambos com $\mathrm{D}$ dimensões, satisfazendo à condição $T_{\mathrm{q}} \mathcal{S} \subset$ $\operatorname{ker} f^{\prime}(\mathrm{q})$, vemos que $T_{\mathrm{q}} \mathcal{S}=\operatorname{ker} f^{\prime}(\mathrm{q})$. 


\section{Apêndice $\mathrm{C}$}

\section{Algumas informações relevantes}

\section{C.1 Uma observação quântica adicional}

Seja um sistema físico definido em alguma variedade Riemanniana $\mathcal{M}$ com $\mathrm{D}$ dimensões, possuindo descrição clássica Hamiltoniana, e para o qual uma teoria quântica se constrói em termos da representação de posição conforme o mencionado no Capítulo 4.

Considerando que $\mathcal{M}$ se parametriza pelos parâmetros intrínsecos $q=\left(q^{1}, \ldots, q^{\mathrm{D}}\right)$ num dos seus abertos $U$, e uma vez que a descrição quântica do sistema físico se faz com o auxílio de um espaço de Hilbert $\mathfrak{H}$ complexo, os estados quânticos a ele associados se descrevem necessariamente por uma função $\varphi: \mathcal{M}_{U} \rightarrow \mathbb{C}$ dada por

$$
\varphi(q)=c^{\mathrm{a}} u_{\mathrm{a}}(q)
$$

onde $c^{\mathrm{a}}$ são coeficientes complexos explicitamente definidos por

$$
c^{\mathrm{a}}=\int u_{\mathrm{a}}^{*}(q) \varphi(q) d q
$$

sendo $\left\{u_{\mathrm{a}}(q)\right\}$ um conjunto de funções quadrado integráveis servindo de base para $\mathfrak{H}$ [34]. Assim por exemplo, o produto interno entre $\varphi$ e outra $\psi: \mathcal{M}_{U} \rightarrow \mathbb{C}$ dá-se por

$$
\langle\psi, \varphi\rangle=\int \psi^{*}(q) \varphi(q) w(q) d q
$$

onde $w: \mathcal{M}_{\mathbf{U}} \rightarrow \mathbb{R}_{+}[2]$.

Com a adoção de outro conjunto de parâmetros $q^{\prime}=\left(q^{\prime 1}, \ldots, q^{\prime \mathrm{D}}\right)$ também capazes de descrever 
$\mathcal{M}_{\mathrm{U}}$, através dos quais as funções $\varphi^{\prime}, \psi^{\prime}: \mathcal{M}_{\mathbf{U}} \rightarrow \mathbb{C}$ e $w^{\prime}: \mathcal{M}_{\mathbf{U}} \rightarrow \mathbb{R}_{+}$analogamente remontam a

$$
\left\langle\psi^{\prime}, \varphi^{\prime}\right\rangle=\int \psi^{\prime *}\left(q^{\prime}\right) \varphi^{\prime}\left(q^{\prime}\right) w^{\prime}\left(q^{\prime}\right) d q^{\prime}
$$

observamos, face à invariância do produto interno frente às trocas de parâmetros, que todas as funções supracitadas se relacionam por meio de

$$
\varphi^{\prime}\left(q^{\prime}\right)=\varphi\left(q\left(q^{\prime}\right)\right)=\varphi(q) \quad, \psi^{\prime}\left(q^{\prime}\right)=\psi\left(q\left(q^{\prime}\right)\right)=\psi(q)
$$

sendo

$$
w^{\prime}\left(q^{\prime}\right)=w\left(q\left(q^{\prime}\right)\right)=w(q)
$$

identificável com a raíz quadrada da métrica Riemanniana associada a $\mathcal{M}_{\mathbf{U}}$. Ou seja, tomando $\mathcal{X}(\mathcal{M})$ pelo conjunto de todos os campos vetoriais e $\mathcal{D}(\mathcal{M})$ por um anel com funções reais ${ }^{1}$, ambos definidos na variedade em pauta, ao considerarmos $g: \mathcal{X}(\mathcal{M}) \times\left.\mathcal{X}(\mathcal{M})\right|_{\boldsymbol{U}} \rightarrow \mathcal{D}(\mathcal{M})$ como um tensor métrico relacionado a $\mathcal{M}_{\mathbf{U}}$, o produto escalar (C.1) se expressa por

$$
\langle\psi, \varphi\rangle=\int \psi^{*}(q) \varphi(q) \sqrt{\operatorname{det} g(q)} d q
$$

Este resultado particularmente aponta que, nesta representação, $\mathfrak{H}$ é constituído por funções complexas escalares de módulo integrável segundo a medida Riemanniana $\sqrt{\operatorname{det} g(q)} d q[45]$.

\section{C.2 Outras informações geométricas}

\section{C.2.1 Sobre a métrica e a curvatura}

Consideremos $\mathcal{M}$ agora como uma subvariedade Riemanniana, cuja métrica é dada pelo mesmo tensor supracitado, mergulhada em outra Euclideana parametrizada por $x=\left(x^{1}, \ldots, x^{\mathrm{D}+\mathrm{N}}\right)$. Observando, por exemplo, que os símbolos de Christoffel para $\mathcal{M}_{\mathrm{U}}$ são dados por

$$
\Gamma_{\alpha \beta}^{\mu}=\frac{1}{2} g^{\mu \nu}\left(\frac{\partial g_{\nu \alpha}}{\partial q^{\beta}}+\frac{\partial g_{\nu \beta}}{\partial q^{\alpha}}-\frac{\partial g_{\alpha \beta}}{\partial q^{\nu}}\right)
$$

notamos, especificamente através de (2.6), que

$$
\Gamma_{\alpha \beta}^{\mu}=g^{\mu \nu}\left(\delta_{j \mathrm{k}} \frac{\partial x^{\mathrm{j}}}{\partial q^{\nu}} \frac{\partial^{2} x^{\mathrm{k}}}{\partial q^{\alpha} \partial q^{\beta}}\right)=\frac{\partial q^{\mu}}{\partial x^{\mathrm{j}}} \frac{\partial^{2} x^{\mathrm{j}}}{\partial q^{\alpha} \partial q^{\beta}} \Rightarrow \Gamma_{\alpha \mu}^{\mu}=\frac{\partial q^{\mu}}{\partial x^{\mathrm{j}}} \frac{\partial^{2} x^{\mathrm{j}}}{\partial q^{\mu} \partial q^{\alpha}}
$$

\footnotetext{
${ }^{1}$ No caso, as funções e os campos vetoriais supracitados devem ser considerados de classe $C^{\mathrm{k}}$.
} 
Utilizando uma expressão análoga para o ambiente Euclideano, também segue imediatamente que $\Gamma_{\mathrm{k} 1}^{\mathrm{j}}=0$, haja vista que a métrica $\delta: \mathcal{X}\left(\mathbb{R}^{\mathrm{D}+\mathrm{N}}\right) \times \mathcal{X}\left(\mathbb{R}^{\mathrm{D}+\mathrm{N}}\right) \rightarrow \mathcal{D}\left(\mathbb{R}^{\mathrm{D}+\mathrm{N}}\right)$ a ele associada independe da parametrização.

Outro tensor de extrema importância para uma variedade Riemanniana é o misto de curvatura. No caso específico de $\mathcal{M}_{\mathbf{U}}$, trata-se do tensor $R: \mathcal{X}(\mathcal{M}) \times \mathcal{X}(\mathcal{M}) \times \mathcal{X}(\mathcal{M}) \times\left.\mathcal{X}(\mathcal{M})\right|_{U} \rightarrow \mathcal{D}(\mathcal{M})$ definido por

$$
R_{\mu \nu \beta}^{\alpha}=\Gamma_{\mu \beta, \nu}^{\alpha}-\Gamma_{\mu \nu, \beta}^{\alpha}+\Gamma_{\mu \beta}^{\gamma} \Gamma_{\gamma \nu}^{\alpha}-\Gamma_{\mu \nu}^{\gamma} \Gamma_{\gamma \beta}^{\alpha}
$$

o qual é identicamente nulo quando $\mathcal{M}_{U}$ tratar-se de uma subvariedade cuja métrica independa da parametrização, assim como $\mathbb{R}^{\mathrm{D+N}}$. Aliás, a partir desta mesma expressão, se torna possível expressar o tensor de Ricci e a curvatura invariante por meio das respectivas expressões [12]

$$
R_{\mu \nu}=R_{\mu \nu \alpha}^{\alpha}=\Gamma_{\mu \alpha, \nu}^{\alpha}-\Gamma_{\mu \nu, \alpha}^{\alpha}+\Gamma_{\mu \alpha}^{\gamma} \Gamma_{\gamma \nu}^{\alpha}-\Gamma_{\mu \nu}^{\gamma} \Gamma_{\gamma \alpha}^{\alpha}, \text { e } \mathrm{R}=g^{\mu \nu} R_{\mu \nu}
$$

\section{C.2.2 Alguns resultados específicos}

De acordo com [12], denotando por g o determinante do tensor métrico associado a $\mathcal{M}_{\mathbf{U}}$, observamos que

$$
\frac{\partial \mathrm{g}}{\partial g_{\mu \nu}}=\mathrm{g} g^{\mu \nu} \Rightarrow \frac{\partial}{\partial g_{\mu \nu}} \ln \mathrm{g}=g^{\mu \nu} \Rightarrow \frac{\partial}{\partial q^{\alpha}} \ln \mathrm{g}=\frac{\partial g_{\mu \nu}}{\partial q^{\alpha}} \frac{\partial}{\partial g_{\mu \nu}} \ln \mathrm{g}=\frac{\partial g_{\mu \nu}}{\partial q^{\alpha}} g^{\mu \nu}
$$

Substituindo as expressões (2.6) e (2.10) nesta última igualdade vemos, por exemplo, que

$$
\frac{\partial}{\partial q^{\alpha}} \ln g=\frac{\partial g_{\mu \nu}}{\partial q^{\alpha}} g^{\mu \nu} \Rightarrow \frac{1}{2} \frac{\partial}{\partial q^{\alpha}} \ln g=\frac{\partial q^{\mu}}{\partial x^{j}} \frac{\partial^{2} x^{j}}{\partial q^{\mu} \partial q^{\alpha}}=\Gamma_{\mu \alpha}^{\mu},
$$

em respeito ao resultado (C.2). Aliás, desta mesma relação também obtemos

$$
\frac{1}{4} \frac{\partial}{\partial q^{\alpha}} \ln \mathrm{g}=\frac{\partial}{\partial q^{\alpha}} \ln \mathrm{g}^{\frac{1}{4}}=\mathrm{g}^{-\frac{1}{4}} \frac{\partial \mathrm{g}^{\frac{1}{4}}}{\partial q^{\alpha}}=\frac{1}{2} \Gamma_{\mu \alpha}^{\mu}
$$

Tratam-se de expressões úteis ao corpo do texto principal, necessárias principalmente para o bom desenvolvimento do Capítulo 4. 


\section{Referências Bibliográficas}

[1] N. C. A. da Costa: O Conhecimento Científico (Discurso Editorial, São Paulo, 1997).

[2] L. E. Ballentine: Quantum Mechanics: A Modern Development (World Scientific, Singapore, 2000).

[3] M. Henneaux, C. Teitelboim: Quantization of Gauge Systems (Princeton University Press, Princeton New Jersey 1992).

[4] J.-M. Souriau: Structures des Systemes Dynamiques (Dunod, Paris 1970).

[5] P. Deligne, et all (eds.): Quantum Fields and Strings: A Course for Mathematicians - Volume 1 (American Mathematical Society, Institute for Advanced Study 2000).

[6] M. J. Gotay: International Journal of Theoretical Physics, 19, No. 2, 139, 1980.

[7] D. M. Gitman, I. P. Tyutin: Quantization of Fields with Constraints (Springer-Verlag, Berlin Heidelberg 1990).

[8] J. L. M. Assirati: Quantização Covariante de Sistemas Mecânicos (Tese de Doutorado IFUSP, São Paulo 2010).

[9] H. J. Groenwald: Physica, 12, 405 (1946).

[10] L. van Hove: Proc. Roy. Acad. Sci. Belgium, 26, 1 (1951).

[11] L. P. Eisenhart: Riemannian Geometry (Princeton University Press, New Jersey 1949).

[12] J. L. Synge, A. Shild: Tensor Calculus (Dover Publications Inc., New York 1978).

[13] A. A. Deriglazov, E. J. G. Filgueiras: Formalismo Hamiltoniano e Transformações Canônicas em Mecânica Clássica (Editora Livraria da Física, São Paulo 2009).

[14] B. S. DeWitt: Phys. Rev. 85, 653 (1952). 
[15] M. P. do Carmo: Geometria Diferencial de Curvas e Superfícies (Textos Universitários, Sociedade Brasileira de Matemática, Rio de Janeiro 2008).

[16] I. J. R. Aitchison, A. J. G. Hey: Gauge Theories in Particles Physics, Volume I: From Relativistic Quantum Mechanics to QCD (Institute of Physics Publishing, Bristol and Phyladelphia 2003).

[17] N. Ogawa, K. Fujii, A. Kobushkin: Prog. Theor. Phys. 83, 894 (1990).

[18] N. Ogawa, K. Fujii, N. Chepilko, A. Kobushkin: Prog. Theor. Phys. 85, 1189 (1991).

[19] T. Homma, T. Inamoto, T. Miyazaki: Prog. Theor. Phys. 85, 2049 (1991).

[20] A. Shimizu, T. Inamoto, T. Miyazaki: Nuovo Cimento B 107, 973 (1992).

[21] A. Saa: Class. Quantum Grav. 14, 385 (1997).

[22] E. L. Lima: Variedades Diferenciáveis (Monografia de Matemática, IMPA, Rio de Janeiro 1973).

[23] M. P. do Carmo: Geometria Riemanniana (Projeto Euclides, IMPA, Rio de Janeiro 2005).

[24] P. A. M. Dirac: Lectures on Quantum Mechanics (Yeshiva University Press, New York 1964).

[25] M. Spivak: Calculus on Manifolds: A Modern Approach to Classical Theorems of Advanced Calculus (The Benjamin-Cummings Publishing Company, New York 1965).

[26] A. R. Larotonda: Notas sobre Variedades Diferenciables (Notas de Geometria y Topologia N. 1, Universidad Nacional Del Sur, INMABB - CONICET, Bahia Blanca 1980).

[27] E. Spainer: Mergulhando Variedades Suaves em Espaços Euclideanos (Matemática Universitária $N^{\circ} .9 / 10$, dezembro de 1989).

[28] J. Nash: Ann. of Math., 63, 20 (1956).

[29] E. L. Lima: Álgebra Linear (Coleção Matemática Universitária, IMPA, Rio de Janeiro 2008).

[30] J. M. Lee: Riemannian Manifolds: An Introduction to Curvature (Springer-Verlag, New York 1997).

[31] C. Lanczos: The Variational Principles of Mechanics (University of Toronto Press, Toronto 1952). 
[32] E. L. Lima: Análise Real, Volume II (Coleção Matemática Universitária, IMPA, Rio de Janeiro 2007).

[33] N. Lemos: Mecânica Analítica (Editora Livraria da Física, São Paulo 2007).

[34] K. Gottfried, T.-M. Yan: Quantum Mechanics: Fundamentals, Second Edition (SpringerVerlag, New York Inc. 2003).

[35] S. R. de Groot, I. G. Suttorp: Foundations of Electrodynamics (North-Holland Publishing Company, Amsterdam 1972).

[36] L. D. Landau, E. M. Lifshitz: Mechanics (Pergamon Press, Oxford, New York 1976).

[37] M. O. C. Gomes: Teoria Quântica dos Campos (Edusp, São Paulo 2000).

[38] H. Kleinert, S. Shabanov: Phys. Lett. B 428, 315 (1998).

[39] D. Hoffman, W. Meeks: Science News, 27, No. 11, 161 (1985).

[40] C. Quigg: Gauge Theories of the Strong, Weak and Eletromagnetic Interactions (Advanced Book Classics, Westview Press, Illinois 1997).

[41] K. B. Marathe, G. Martucci: The Mathematical Foundations of Gauge Theories (NorthHolland, Amsterdam 1992).

[42] N. M. J. Woodhouse: Geometric Quantization, Second Edition (Clarendon Press, Oxford 1997).

[43] V. Guillemin, A. Pollack: Differential Topology (Prentice-Hall, Inc., Englewood Cliffs, New Jersey 1974).

[44] K. Hoffman, R. A. Kunze: Linear Algebra (Prentice-Hall, New Delhi 1967).

[45] J. L. M. Assirati: Generalização Covariante da Correspondência de Weyl e Quantização da Partícula no Espaço Curvo (Dissertação de Mestrado IFUSP, São Paulo 2001).

[46] S. Rosenberg: The Laplacian on a Riemannian Manifold (London Mathematical Society Student Texts 31, Cambridge University Press, New York 1998). 